MICHAEL VIRIATO ARAUJO

\title{
SELEÇÃO DINÂMICA DE PORTFÓLIOS EM MÉDIA-VARIÂNCIA COM SALTOS MARKOVIANOS
}

São Paulo

2007 
MICHAEL VIRIATO ARAUJO

\section{SELEÇÃO DINÂMICA DE PORTFÓLIOS EM MÉDIA-VARIÂNCIA COM SALTOS MARKOVIANOS}

Tese apresentada ao Departamento de Engenharia de Telecomunicações e Controle da Universidade de São Paulo para obtenção do título de Doutor em Engenharia Elétrica.

Área de Concentração: Engenharia de Sistemas Orientador: Prof. Dr. Oswaldo Luiz do Valle Costa

São Paulo

2007 
FICHA CATALOGRÁFICA

Araujo, Michael Viriato

Seleção dinâmica de portfólios em média-variância com saltos markovianos / M.V. Araujo. -- São Paulo, 2007.

$146 \mathrm{p}$.

Tese (Doutorado) - Escola Politécnica da Universidade de São Paulo. Departamento de Engenharia de Telecomunicações e Controle.

1.Controle estocástico 2.Sistemas discretos 3.Administração de carteiras 4.Processos de Markov 5.Administração de portfólio I.Universidade de São Paulo. Escola Politécnica. Departamento de Engenharia de Telecomunicações e Controle Il.t. 


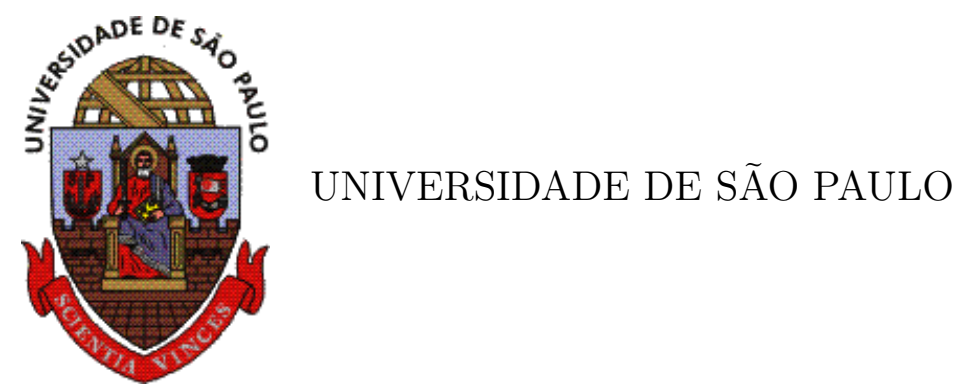

FOLHA DE APROVAÇÃO

\section{Seleção Dinâmica de Portfólios em Média-Variância com Saltos Markovianos}

\section{MICHAEL VIRIATO ARAUJO}

Tese apresentada ao Departamento de Engenharia de Telecomunicações e Controle da Universidade de São Paulo para obtenção do título de Doutor em Engenharia Elétrica.

Área de Concentração: Engenharia de Sistemas

Banca Examinadora constituída por:

Dr. Oswaldo Luiz do Valle Costa - Orientador Universidade de São Paulo

Dra. Celma de Oliveira Ribeiro

Universidade de São Paulo

Dr. FuAd Kassab Junior

Universidade de São Paulo

Dr. JoÃo Bosco RiBeiro do VAL

Universidade Estadual de Campinas

Dr. TAKASHI YONEYAMA

Instituto Tecnológico da Aeronáutica

São Paulo, 2007 


\section{Dedicatória}

A Jane Araújo Ferreira, minha esposa, pela sua compreensão, incentivo e apoio fundamentais para a conclusão deste trabalho.

A minha família, Haroldo Euclides de Araújo, Alice Maria Viriato Araújo, Haroldo E. A. Júnior, Rodrigo V. Araújo e sobrinhos, que mesmo distantes acompanharam e estimularam a realização desta obra. 


\section{Agradecimentos}

Ao Professor Dr. Oswaldo Luiz do Valle Costa pelo que muito me ensinou, pela oportunidade única de desenvolver este trabalho e pela paciência e disponibilidade na orientação desta Tese. A experiência adquirida ao longo do curso de doutorado foi sem dúvida engrandecedora para meu desenvolvimento pessoal e profissional.

À Escola Politécnica da USP pela oportunidade de realização do curso de doutorado e a todos os professores do curso pelos ensinamentos transmitidos.

Não posso deixar de citar a relevante contribuição do Banco Itaú S.A. pelo incentivo e disponibilização de tempo para realização deste trabalho. 
"There is nothing so disastrous as a rational investment policy in an irrational world".

John Maynard Keynes 


\section{Resumo}

Investiga-se, em tempo discreto, o problema multi-período de otimização de carteiras generalizado em média-variância cujos coeficientes de mercado são modulados por uma cadeia de Markov finita. O problema multi-período generalizado de média-variância com saltos Markovianos (PGMV) é um problema de controle estocástico sem restrição cuja função objetivo consiste na maximização da soma ponderada ao longo do tempo da combinação linear de três elementos: o valor esperado da riqueza do investidor, o quadrado da esperança desta riqueza e a esperança do quadrado deste patrimônio. A principal contribuição deste trabalho é a derivação analítica de condições necessárias e suficientes para a determinação de uma estratégia ótima de investimento para o problema $P G M V$. A partir deste modelo são derivadas várias formulações de médiavariância, como o modelo tradicional cujo objetivo é maximizar o valor esperado da riqueza final do investidor, dado um nível de risco (variância) do portfólio no horizonte de investimento, bem como o modelo mais complexo que busca maximizar a soma ponderada das esperanças da riqueza ao longo do tempo, limitando a perda deste patrimônio em qualquer momento. Adicionalmente, derivam-se formas fechadas para a solução dos problemas citados quando as restrições incidem somente no instante final. Outra contribuição deste trabalho é a extensão do modelo $P G M V$ para a solução do problema de seleção de carteiras em média-variância com o objetivo de superar um benchmark estocástico, com restrições sobre o valor esperado ou sobre a variância do tracking error do portfólio. Por fim, aplicam-se os resultados obtidos em exemplos numéricos cujo universo de investimento são todas as ações do IBOVESPA. 


\section{Abstract}

In this work we deal with a discrete-time multi-period mean-variance portfolio selection model with the market parameters subject to Markov regime switching. The multi-period generalized mean-variance portfolio selection model with regime switching $(P G M V)$ is an unrestricted stochastic control problem, in which the objective function involves the maximization of the weighted sum of a linear combination of three parts: the expected wealth, the square of the expected wealth and the expected value of the wealth squared. The main contribution of this work is the analytical derivation of necessary and sufficient conditions for the existence of an optimal control strategy to this $P G M V$ model. We show that several mean-variance models are derived from the $P G M V$ model, as the traditional formulation in which the objective is to maximize the expected terminal wealth for a given final risk (variance), or the complex one in which the objective function is to maximize the weighted sum of the wealth throughout its investment horizon, with control over maximum wealth lost. Additionally, we derive closed forms solutions for the above models when the restrictions are just in the final time. Another contribution of this work is to extend the $P G M V$ model to solve the multi-period portfolio selection problem of beating a stochastic benchmark with control over the tracking error variance or its expected value. Finally, we run numerical examples in which the investment universe is formed by all the stocks belonging to the IBOVESPA. 


\section{Sumário}

1 Introdução

2 Revisão Bibliográfica 6

3 Descrição do Mercado e Formulação dos Problemas $\quad 15$

3.1 Descrição do Mercado . . . . . . . . . . . . . . . . . . . 15

3.2 O Portfólio . . . . . . . . . . . . . . . . . . . . . . 18

3.3 Formulação do Problema Generalizado de Média-Variância e dos Modelos Derivados ........................... 21

3.3.1 Formulação para o Problema Generalizado de Média-Variância com Saltos Markovianos . . . . . . . . . . . . . 25

3.3.2 Formulação do Problema Auxiliar . . . . . . . . . . . . . . . . 28

4 Solução para o Problema Generalizado de Média-Variância com Parâmetros Sujeitos a Saltos Markovianos $\quad 30$

4.1 Lei de Controle Ótima . . . . . . . . . . . . . . . . . . 30

4.2 Resultados da Formulação Auxiliar . . . . . . . . . . . . . . . . . 36

4.3 Solução para o Problema Generalizado de Média-Variância . . . . . . . 41

4.3 .1 Condição Necessária . . . . . . . . . . . . . . . . 42

4.3.2 Condição Suficiente . . . . . . . . . . . . . . . . . . . . . . 47

5 Aplicação do $P G M V$ a Problemas Específicos de Média-Variância $\quad 56$ 
5.1 Solução para os Problemas de Média-Variância com Custo e Controle nos Períodos Intermediários . . . . . . . . . . . . . . . 57

5.2 Solução para os Problemas de Média-Variância com Custo nos Períodos Intermediários e Restrição apenas no Instante Final . . . . . . . . . . . 60

5.3 Solução para os Problemas Multi-Período Tradicionais de Média-Variância com Parâmetros Sujeitos a Saltos Markovianos . . . . . . . . . . . . 68

5.4 Modelo no qual o Mercado é Formado por $n$ Ativos de Risco e um Ativo Livre de Risco . . . . . . . . . . . . . . . . . . . . . 70

5.4.1 Simplificações no Mercado . . . . . . . . . . . . . . . . . . 70

5.4.2 Solução para os Problemas no Caso em que Existe um Ativo Livre de Risco . . . . . . . . . . . . . . . . . . 75

6 Seleção de Carteiras para Superar um Benchmark $\quad 77$

6.1 O Tracking Error do Portfólio . . . . . . . . . . . . . . 78

6.2 Formulação do Problema Generalizado de Média-Variância do Tracking Error com Saltos Markovianos . . . . . . . . . . . . . . 81

6.2.1 Formulações Derivadas do PGMVTE . . . . . . . . . . 82

6.3 Solução do Problema PGMVTE . . . . . . . . . . . . . . . . . . 84

6.4 Solução dos Problemas Derivados do PGMVTE . . . . . . . . 85

6.4.1 Solução para os Problemas com Custo e Restrições Intermediárias 85

6.4.2 Solução para os Problemas com Custo Intermediário e Restrições apenas no Instante Final . . . . . . . . . . . . . . . . . . . . 86

6.4.3 Solução para os Problemas com Custo e Restrições apenas no Instante Final . . . . . . . . . . . . . . . . . . . . . 88

$\begin{array}{lll}7 & \text { Exemplos Numéricos } & 91\end{array}$

7.1 Mercado apenas com Ativos de Risco . . . . . . . . . . . . . . . . . 91

7.2 Mercado no qual Existe um Ativo Livre de Risco . . . . . . . . . . . 98

7.3 Otimização de Carteira para Superar um Benchmark . . . . . . . . . . 104 
Referências Bibliográficas 


\section{Lista de Figuras}

7.1 Fronteiras Eficientes (Esperança da Riqueza Final $x$ Variância do Patrimônio Final) - Mercado formado apenas por ativos de risco brasileiros. . . . . . . 96

7.2 Fronteiras Eficientes (Esperança da Riqueza Final $x$ Variância do Patrimônio Final) - Mercado formado apenas com ativos de risco brasileiros e com vetor de probabilidade de estado $\pi(t)=(40 \%, 60 \%)^{\prime}$. . . . . . . . . .

7.3 Fronteiras Eficientes (Esperança da Riqueza Final $x$ Variância do Patrimônio Final) - Mercado formado apenas com ativos de risco brasileiros e com vetor de probabilidade de estado $\pi(t)=(80 \%, 20 \%)^{\prime}$. . . . . . . . . . . . . . 101

7.4 Fronteiras Eficientes (Esperança da Riqueza Final $x$ Variância do Patrimônio Final) - Mercado formado por ativos de risco e um livre de risco. . . . . . . 103

7.5 Fronteiras Eficientes (Valor Esperado do Tracking Error Final $x$ Variância do Tracking Error Final) para os problemas de seleção de carteiras para superar um benchmark, com controle sobre a volatilidade do tracking error (variância do tracking error). . . . . . . . . . . . . . . . . . 107 


\section{Lista de Tabelas}

7.1 Resultados das simulações de Monte Carlo para o problema P . . . . . . 96

7.2 Resultados das simulações de Monte Carlo para o problema PMV . . . . . 97

7.3 Resultados das simulações de Monte Carlo para o problema $P B C$. . . . 97

7.4 Resultados das simulações de Monte Carlo para o problema $\mathrm{P}_{\mathrm{SM}}$. . . . . . 97

7.5 Resultados das simulações de Monte Carlo para o problema P . . . . . . . 99

7.6 Resultados das simulações de Monte Carlo para o problema PMV . . . . . 100

7.7 Resultados das simulações de Monte Carlo para o problema PBC . . . . . 100

7.8 Resultados das simulações de Monte Carlo para o problema PBC . . . . . 101

7.9 Resultados das simulações de Monte Carlo para o problema PMV . . . . . 102

7.10 Resultados das simulações de Monte Carlo para o problema $P B C$. . . . 102

7.11 Resultados das simulações de Monte Carlo para o problema P . . . . . . 103

7.12 Resultados das simulações de Monte Carlo para o problema PMV . . . . . 104

7.13 Resultados das simulações de Monte Carlo para o problema PBC . . . . 104

7.14 Resultados das simulações de Monte Carlo para o problema Psm . . . . . 104

7.15 Resultados das simulações para o problema PMVTE . . . . . . . . 106

7.16 Resultados das simulações para o problema PMVTE $E_{s m}$. . . . . . . . 106

7.17 Carteiras para o Primeiro Período no Problema de Otimização de Carteiras com Ativos de Risco apenas. . . . . . . . . . . . . . . . . . . . . . . . . . 108

7.18 Carteiras para o Primeiro Período no Problema de Otimização de Carteiras com um Ativo Livre de Risco. . . . . . . . . . . . . . . . . . . . . . . . 109 
7.19 Carteiras para o Primeiro Período no Problema de Otimização de Carteira para Superar um Benchmark. . . . . . . . . . . . . . . . . . . 110

7.20 Tabela com Ativos de Risco, seus códigos Bloomberg e retornos médios trimestrais. . . . . . . . . . . . . . . . . . 11

7.21 Matriz de covariância dos ativos de risco em todo o período amostral (2000 a 2006)(Parte I). . . . . . . . . . . . . . . . . . . . . . 112

7.22 Matriz de covariância dos ativos de risco em todo o período amostral (2000 a 2006)(Parte II) . . . . . . . . . . . . . . . . . . . . . 113

7.23 Matriz de covariância dos ativos de risco em todo o período amostral (2000 a 2006)(Parte III). . . . . . . . . . . . . . . . . . . . . . 114

7.24 Matriz de covariância dos ativos de risco em todo o período amostral (2000 a 2006)(Parte IV).

7.25 Matriz de covariância dos ativos de risco para o cenário com tendência positiva no período amostral (2000 a 2006)(Parte I). . . . . . . . . . . . . . 116

7.26 Matriz de covariância dos ativos de risco para o cenário com tendência positiva no período amostral (2000 a 2006)(Parte II). . . . . . . . . . . . . 117

7.27 Matriz de covariância dos ativos de risco para o cenário com tendência positiva no período amostral (2000 a 2006)(Parte III). . . . . . . . . . . . 118

7.28 Matriz de covariância dos ativos de risco para o cenário com tendência positiva no período amostral (2000 a 2006)(Parte IV). . . . . . . . . . . . . 119

7.29 Matriz de covariância dos ativos de risco para o cenário com tendência negativa no período amostral (2000 a 2006)(Parte I)

7.30 Matriz de covariância dos ativos de risco para o cenário com tendência negativa no período amostral (2000 a 2006)(Parte II) . . . . . . . . . . . .

7.31 Matriz de covariância dos ativos de risco para o cenário com tendência negativa no período amostral (2000 a 2006)(Parte III) . . . . . . . . . . . . 122

7.32 Matriz de covariância dos ativos de risco para o cenário com tendência negativa no período amostral (2000 a 2006)(Parte IV). 


\section{Capítulo 1}

\section{Introdução}

A pesquisa em otimização de portfólios de investimentos se destaca pela relevância econômica dos impactos que as decisões de grandes investidores, como os fundos de pensão, exercem sobre economias de empresas e até de países. Uma administração não eficiente dos ativos de um fundo de pensão pode provocar prejuízos para todo um conjunto de entidades relacionadas a ele: prejuízos para a aposentadoria de seus beneficiários, para suas empresas patrocinadoras, mediante a elevação de sua contribuição periódica, perdas financeiras aos acionistas das empresas patrocinadoras, risco de imagem e prejuízos financeiros para o administrador destes recursos.

A pesquisa em otimização de carteiras evoluiu de forma relevante a partir do trabalho pioneiro de Markowitz (1952). Desde então, o desafio de estudo nesta área tem sido o de adaptar os modelos financeiros de forma a se aproximarem cada vez mais ao real ambiente que os administradores de recursos enfrentam.

Neste contexto, como uma forma de ampliar as fontes de incerteza nos modelos financeiros e assim capturar uma classe mais ampla de fenômenos que permeiam os mercados de capitais, tem havido um crescente interesse no estudo de modelos financeiros nos quais os parâmetros chave (taxa de retorno e volatilidade dos 
preços) são modulados por uma cadeia de Markov, por exemplo, em (Zhang (2000)), (Cajueiro (2002)), (Bauerle e Rieder (2004)), (Zhou e Yin (2003)), (Yin e Zhou (2004)) e (Çakmak e Özekici (2006)). Tais modelos refletem de forma mais apropriada o movimento do mercado, já que, usualmente os ativos oscilam seguindo uma tendência geral ditada pelo estado da economia, pelo humor dos investidores ou por outro evento global.

Nesta tese, propõe-se um modelo generalizado de otimização multi-período de carteiras em média-variância com os coeficientes de mercado modulados por uma cadeia de Markov finita, o qual será denotado de $P G M V$. O modelo $P G M V$ pode ser visto como um problema de controle estocástico no qual a função objetivo consiste da soma ponderada ao longo do tempo de uma combinação linear entre três elementos: o valor esperado da riqueza do investidor, o quadrado da esperança desta riqueza e a esperança do quadrado deste patrimônio.

Demonstra-se que a partir do modelo $P G M V$, derivam-se várias formulações de média-variância. Dentre estas formulações, três são objetivo de análise neste trabalho:

I a formulação multi-período tradicional de média-variância, que pode ser posta em duas formas: aquela cuja função objetivo é maximizar a riqueza final esperada dado um nível de risco para o instante final (variância da riqueza final) $\left(P\left(\sigma^{2}\right)\right)$, ou minimizar a variância do patrimônio terminal do investidor para um desejado valor esperado deste patrimônio final $(P(\epsilon))$;

II a formulação multi-período de média-variância, que também pode ser posta em duas formas: uma em que a função objetivo é a maximização da soma ponderada do valor esperado da riqueza ao longo do tempo, tendo como restrição o risco (variância do patrimônio) em cada instante de tempo $\left(P M V\left(\sigma^{2}\right)\right)$, ou outra em que a função objetivo é minimizar a soma ponderada da variância da riqueza ao longo do tempo, tendo como restrição a esperança do patrimônio em cada momento $(P M V(\epsilon))$;

III a formulação com controle sobre perda máxima, cuja função objetivo é maximi- 
zar a soma ponderada do valor esperado da riqueza ao longo do tempo, tendo como restrição a probabilidade máxima $(\varrho)$ de o patrimônio cair abaixo de um determinado valor $(\psi)$ em cada instante, denotada por $P B C(\varrho, \psi)$.

A principal contribuição deste trabalho é a derivação de condições necessárias e suficientes para obtenção de uma política ótima de controle para o problema $P G M V$. Recorda-se a diferença entre uma condição necessária e uma condição suficiente. Uma condição necessária é aquela que deve ser satisfeita para que a afirmação seja verdadeira. Enquanto a condição suficiente é aquela que se satisfeita garante que a afirmação é verdadeira. Assim, apresenta-se um algoritmo de condição necessária e um algoritmo de condição suficiente para determinação de uma estratégia ótima de controle para o problema $P G M V$. Portanto, para o algoritimo de condição necessária, mostra-se que se a estratégia de investimento é ótima, então ela atende a uma determinada condição. Para o segundo algoritimo, mostra-se que se as condições suficientes são satisfeitas, então a estratégia de investimento obtida é ótima.

Demonstra-se que esta estratégia ótima de investimentos é obtida através de um procedimento recursivo baseado em um conjunto de equações a diferenças de Riccati interconectadas e em um segundo conjunto de equações recursivas.

Destaca-se que não se tem conhecimento sobre trabalhos anteriores que derivaram uma condição suficiente para determinação da solução ótima a tempo discreto de problemas multi-período de seleção de carteiras em média-variância com saltos Markovianos. Além disso, também não se tem conhecimento de trabalhos anteriores que tenham derivado uma condição necessária para o modelo generalizado de média-variância aqui proposto.

Através da solução do problema $P G M V$ é possível encontrar a solução dos demais problemas citados. Mostra-se que para as formulações II e III, quando as restrições também incidem nos períodos intermediários, após a derivação da solução do problema $P G M V$ ainda é necessário um procedimento numérico que objetiva encontrar um conjunto de Multiplicadores de Lagrange que atendam às restrições dos respectivos 
problemas.

Para os casos em que a restrição incide apenas no período final, uma solução em forma fechada pode ser derivada analiticamente. Portanto, para o caso em que todos os ativos do universo de investimento são de risco, são derivadas formas fechadas para a solução da formulação I e II, quando a restrição incide apenas no instante final. Para a formulação I, também é derivada uma solução analítica no caso em que existe um ativo livre de risco, que não sofre a influência de nenhuma fonte de incerteza. Mostra-se que para este último caso, as expressões analíticas para o valor esperado da riqueza final, bem como para a variância final deste patrimônio e para a fronteira eficiente de investimentos apresentam simplificações em relação ao modelo quando todos os ativos são de risco.

Uma abordagem alternativa ao modelo até aqui apresentado, que busca otimizar uma carteira tendo em vista um retorno absoluto é a de otimizá-la com a finalidade de superar um benchmark. A relevância desta nova abordagem resulta do fato dos profissionais de investimento estarem usualmente mais preocupados com o desempenho de suas carteiras em relação a um determinado benchmark do que com seu retorno absoluto. Isto ocorre, pelo fato de que é o desempenho adicional de suas carteiras em relação a um benchmark que define a remuneração destes gestores.

Portanto, outra contribuição desta tese é a de estender a investigação em período simples do modelo de média-variância do tracking error introduzida por Roll (1992) para o modelo em multi-período de seleção de carteiras generalizado de média-variância do tracking error, cujos parâmetros de mercado estão sujeitos a saltos Markovianos, denotado por PGMVTE. Demonstra-se como estender os resultados encontrados para o modelo $P G M V$ para determinar a solução do problema PGMVTE. Adicionalmente, reformulam-se os modelos I a III acima para o enfoque do tracking error, e demonstrase que para estas formulações, quando as restrições incidem apenas no instante final, as soluções analíticas derivadas anteriormente são simplificadas quando utilizado o enfoque do tracking error. 
Destaca-se que os resultados encontrados nos Capítulos 4 a 7 são todos inéditos.

Esta tese está organizada da seguinte forma. No Capítulo 2, realiza-se uma revisão bibliográfica, mostrando a evolução dos modelos de média-variância e as diferenças entre os trabalhos anteriores e esta tese. O mercado em estudo é apresentado no Capítulo 3, juntamente com a formulação do problema $P G M V$ e dos problemas derivados deste. A solução para o problema $P G M V$ é analiticamente obtida no Capítulo 4. Neste capítulo também são estabelecidas condições necessárias e suficientes para a determinação desta solução. No Capítulo 5, apresenta-se as leis de controle ótimas para os problemas propostos. Também neste capítulo, quando nestes modelos as restrições incidem apenas no instante final, encontram-se expressões explícitas para as fronteiras eficientes de investimentos nos casos com e sem a existência de um ativo livre de risco. No Capítulo 6, estende-se os resultados obtidos para o problema $P G M V$ na derivação da solução do modelo PGMVTE e de modelos a este derivados. O benefício alcançado pelos presentes modelos em relação aos que não estão sujeitos a saltos é ilustrado através de exemplos numéricos no Capítulo 7. Conclui-se este trabalho no Capítulo 8 com as possíveis ampliações de estudos e considerações finais. 


\section{Capítulo 2}

\section{Revisão Bibliográfica}

A pesquisa para encontrar a melhor combinação entre risco e retorno em problemas de seleção de investimentos teve seu ponto de inflexão com o pioneiro trabalho de Markowitz (1952). O reconhecimento dos benefícios da diversificação não são recentes, sendo identificados já no artigo de Daniel Bernoulli em 1738 sobre o Paradoxo de St. Petersburg quando este cita em (Bernoulli (1954)):

"... it is advisable to divide goods which are exposed to some small danger into several portions rather than to risk them all together ...",

e por William Shakespeare em O Mercador de Veneza, ambos lembrados pelo próprio Markowitz (1999). Entretanto, embora os benefícios da diversificação já fossem conhecidos, foram os trabalhos de Markowitz (1952) e Roy (1952) que primeiro propuseram formas de quantificar os efeitos da diversificação sobre os investimentos.

A formulação original de média-variância em período simples, apresentada em (Markowitz (1952)), consiste em selecionar um portfólio que apresente o maior valor esperado para a riqueza final $(E(V(T)))$ para um nível de risco desejado neste mesmo instante (variância da riqueza final - $\operatorname{Var}(V(T))$ ), ou encontrar uma carteira que 
apresente a menor variância do patrimônio terminal $(\operatorname{Var}(V(T)))$ para um desejado valor esperado deste patrimônio final $(E(V(T)))$. Vale recordar que, como o modelo é de período simples, o momento final é o próximo período. Formalmente, estas duas formulações, podem ser expressas, respectivamente, como:

$$
\begin{gathered}
\max E(V(T)) \\
\text { sujeito a }: \quad \operatorname{Var}(V(T)) \leq \sigma^{2}, \quad T=1
\end{gathered}
$$

e

$$
\begin{gathered}
\min \operatorname{Var}(V(T)) \\
\text { sujeito a }: \quad E(V(T)) \geq \epsilon, \quad T=1 .
\end{gathered}
$$

Apesar de vastamente utilizado, o modelo original de média-variância tem recebido críticas desde sua introdução, como em (Gressis et al. (1976)) e (Campbell e Viceira (2001)). Recentemente, estas críticas têm se concentrado sobre a utilização da variância como medida de risco na otimização de portfólios, pois ela não considera de forma apropriada a característica de "caudas gordas"de algumas distribuições e por penalizar uniformemente os desvios positivos e negativos, ver (Artzner et al. (1999)), (Rockafellar et al. (2006)), (Ortobelli et al. (2005)) e (Fabozzi et al. (2007)).

Artzner et al. (1999) propuseram quatro axiomas que definem as condições necessárias para uma medida de risco ser "coerente". Apesar do desvio padrão não ser considerado uma medida coerente de risco segundo Artzner et al. (1999), ele se qualifica como uma medida de dispersão pelos axiomas definidos em Rockafellar et al. (2006) e por Ortobelli et al. (2005). De fato, como reconhecido por Fabozzi et al. (2007), nenhuma medida de risco única conseguiria capturar todas as preferências de um investidor e a busca por esta medida de risco ideal talvez nunca termine.

Na verdade, a discussão sobre qual a formulação mais adequada para otimizar um portfólio não é recente, e os estudos sobre funções utilidades diferentes da média- 
variância a antecedem. Como citado por Roy (1952), antes de Markowitz (1952) já haviam estudos em otimização de carteiras envolvendo a maximização de utilidades esperadas. Entretanto, a difusão dos modelos que maximizam uma função utilidade genérica da riqueza final se intensificou através do trabalho de Pratt (1964), que introduziu a quantificação da aversão ao risco do investidor, utilizando a relação entre a segunda e a primeira derivada de sua função utilidade, e dos trabalhos de Merton (1969) e Samuelson (1969), que, respectivamente, otimizaram em tempo contínuo e discreto funções utilidades côncavas do consumo e investimento. A partir de então, como observado por Zhou e Li (2000) e Zhou e Yin (2003), a pesquisa em otimização de portfólios tem sido dominada pelos modelos que maximizam funções utilidades diferentes da média-variância.

Contudo, como citado por Markowitz (2000), Zhou e Li (2000) e Zhou e Yin (2003), o enfoque da média-variância se destaca em relação à maximização de outras funções utilidades pela facilidade computacional, pela dificuldade de se determinar a função utilidade que melhor se adapta ao perfil do investidor e por apresentar de forma mais explícita ao investidor as possíveis relações ótimas entre risco e retorno. Além destes benefícios, Markowitz (1970) demonstra que, se a função utilidade de um investidor pode ser aproximada por uma quadrática, um dos portfólios encontrados na fronteira eficiente terá um retorno próximo ao encontrado na maximização desta função utilidade. O mesmo autor em (Markowitz (2000)) também apresenta uma relação de trabalhos que encontraram resultados semelhantes comparando os dois modelos. Em estudo mais recente, Zhao e Ziemba (2000) concluíram que os resultados obtidos pela média-variância são superiores se o preço realizado dos ativos se situar próximo de seu valor esperado e, inferiores, se a realização ocorrer nas caudas da distribuição.

Não demorou muito e logo se percebeu que os modelos de período simples não eram adequados, já que os investidores usualmente promovem alterações em suas carteiras antes do instante final e que os efeitos das decisões iniciais afetam futuras decisões, portanto, induzindo estes modelos a serem estendidos para uma abordagem multi-período. 
Em vista disso, problemas de seleção de portfólios em multi-período têm sido investigados desde a década de 60, ver (Tobin (1965)), (Merton (1969)), (Samuelson (1969)), (Mossin (1968)), (Ingersoll (1987)), (Karatzas (1997)) e (Pliska (1997)). Entretanto, devido à dificuldade de se resolver diretamente os problemas de média-variância em multi-período, as pesquisas se concentraram na otimização de funções utilidades diferentes da formulação de Markowitz.

A formulação multi-período tradicional de média-variância é igual aos modelos originais (2.1) e (2.2). A diferença reside na necessidade de se obter um conjunto de portfólios intermediários que levem à otimização da função objetivo, em vez de apenas uma carteira como na formulação original de período simples. Uma forma de resolver o problema multi-período de média-variância é através da programação dinâmica. Entretanto, verifica-se que na função objetivo dos problemas de média-variância há um termo do tipo $E(V(T))^{2}$ oriundo da expansão da expressão da variância, ou seja, $\operatorname{Var}(V(T))=E\left(V(T)^{2}\right)-E(V(T))^{2}$. Como descrito por Zhou e Li (2000), a existência na função objetivo do termo $E(V(T))^{2}$, ou de forma mais ampla, do termo $U(E(\cdot))$, onde $U$ é uma função utilidade não linear, dificulta a aplicação da programação dinâmica. Apenas recentemente, em (Li e Ng (2000)), foi introduzida uma técnica para solucionar esta dificuldade. Associando ao problema principal um problema auxiliar, ao qual é possível aplicar a teoria da programação dinâmica, Li e Ng (2000) resolveram analiticamente em tempo discreto o problema de otimização multi-período de carteiras em média-variância com coeficientes determinísticos.

A abordagem introduzida por $\operatorname{Li~e~} \mathrm{Ng}$ (2000) inspirou este trabalho e várias outras estensões, como (Zhou e Li (2000)), (Zhou e Yin (2003)), (Yin e Zhou (2004)), (Leippold et al. (2004)), (Zhu et al. (2004)), (Costa e Nabholz (2005)), (Bielecki et al. (2005)), (Araujo e Costa (2006a)), (Araujo e Costa (2006b)), (Çakmak e Özekici (2006)), (Costa e Araujo (2007a)) e (Costa e Araujo (2007b)). Em (Zhou e Li (2000)), por exemplo, o modelo de $\mathrm{Li}$ e $\mathrm{Ng}$ (2000) foi analisado em tempo contínuo, aplicando-se a teoria de controle linear quadrático estocástico. 
Além da ampliação para multi-período, outras evoluções ao modelo inicial de Markowitz também foram propostas, como por exemplo a utilização de custos de transação em (Goldsmith (1976)) e (Dumas e Luciano (1991)). Entretanto, tão importante quanto a estrutura do modelo, a modelagem do retorno dos ativos se destaca como um dos elementos chave para a obtenção do portfólio ótimo.

Um dos pontos fundamentais para a modelagem na seleção de carteiras é a simulação da incerteza dos mercados financeiros. Esta incerteza se reflete nos modelos através da evolução dos preços dos ativos. Até recentemente, os preços dos ativos eram modelados de forma ainda semelhante ao dos primeiros modelos. Como em (Merton (1969)), (Ingersoll (1987)), (Karatzas (1997)), (Pliska (1997)), (Campbell e Viceira (2001)), (Li e Ng (2000)), (Zhou e Li (2000)) e (Zhu et al. (2004)), o processo de evolução dos preços segue um processo estocástico usualmente composto de dois parâmetros determinísticos: o retorno esperado $(\mu)$ e a volatilidade $(\sigma)$. Dessa forma, em tempo contínuo e em tempo discreto, estes trabalhos modelam o preço de um ativo $i\left(\mathrm{~S}_{i}\right)$, respectivamente, conforme os processos abaixo:

$$
\begin{aligned}
& d \mathrm{~S}_{i}(t)=\mathrm{S}_{i}(t)\left\{\mu_{i}(t) d t+\sum_{j=1}^{n} \sigma_{i j}(t) d W^{j}(t)\right\}, \\
& \mathrm{S}_{i}(t+1)=\mathrm{S}_{i}(t)\left\{1+\mu_{i}(t)+\sum_{j=1}^{n} \sigma_{i j}(t) W^{j}(t)\right\}, \quad t \in[0, T],
\end{aligned}
$$

onde $W(t)=\left(W^{1}(t), \ldots, W^{n}(t)\right)$ representa um movimento Browniano em $\mathbb{R}^{n}$, para o caso contínuo, ou um vetor de variáveis aleatórias independentes e identicamente distribuídas, para o caso discreto.

Utilizando estas formulações para o processo dos preços, estes modelos se adequam bem apenas por um período relativamente curto de tempo e não conseguem responder às mudanças no padrão do movimento do mercado. Isto ocorre, pois as constantes flutuações nos mercados provocam alterações no retorno esperado $(\mu)$ e na volatilidade $(\sigma)$, invalidando as estimativas anteriores destes parâmetros. Na tentativa de superar este problema, alguns autores, como Hull (1997), Lim e Zhou (2002) e Bielecki et al. (2005), evoluíram os modelos financeiros para o caso em que os parâmetros ( $\mu$ e $\sigma$ ) tam- 
bém são randômicos, seguindo processos estocásticos adicionais. Lim e Zhou (2002), por exemplo, estenderam o modelo de Zhou e Li (2000), citado acima, para o caso em que os coeficientes são randômicos. Entretanto, estes modelos também se comportam adequadamente apenas no curto prazo, falhando em capturar movimentos de descontinuidade que modificam de forma drástica a evolução dos preços.

Como descrito por Zhang (2000), uma das importantes características do mercado é o fato dos ativos evoluírem seguindo tendências mestras. Estas tendências são ditadas por diversos aspectos: o humor dos investidores, a situação geral da economia ou por eventos de descontinuidade como os recentes ataques terroristas, as crises ocorridas nos países asiáticos (1998) ou a queda das bolsas de valores (1929 e 1987). Portanto, uma melhor abordagem para simular a incerteza nos mercados é a utilização do processo estocástico para modelar as flutuações normais dos preços, conjuntamente com um processo de Markov, modulando os parâmetros de mercado (retorno esperado e volatilidade), para modelar os movimentos de mudança de tendência ou de descontinuidade. Desta forma, o processo de evolução dos preços de um ativo $i$ é modelado em tempo discreto como:

$$
\mathrm{S}_{i}(t+1)=\mathrm{S}_{i}(t)\left\{1+\mu_{i}(t, \theta(t))+\sum_{j=1}^{n} \sigma_{i j}(t, \theta(t)) W^{j}(t)\right\}, \quad t \in[0, T],
$$

onde $\theta(t)$ é uma cadeia finita de Markov representando as possíveis tendências do mercado citadas acima.

Esta última formulação permite com que a simulação do retorno dos ativos consiga refletir de forma mais adequada o ambiente de incerteza dos mercados financeiros e, assim, proporcionar um melhor resultado para a otimização de carteiras. Outra vantagem desta forma de modelagem dos preços, conforme citado por (Fabozzi et al. (2007)), é que ela minimiza o problema do modelo de média-variância em lidar de forma mais apropriada com a característica de "caudas gordas"de algumas distribuições.

Modelos financeiros que se utilizam de cadeias de Markov para modular os parâmetros de mercado têm sido frequentemente utilizados no apreçamento de opções, por 
exemplo (Masi et al. (1994)), (Fouque et al. (2000)) e (Buffington e Elliott (2002)). Recentemente, Zhang (2000) também utilizou esta abordagem para encontrar uma estratégia ótima de venda de ativos.

No processo de otimização de carteiras este enfoque ainda é incipiente. Em (Cajueiro (2002)), os autores aprimoraram o clássico trabalho de Merton (1969), sujeitando as variáveis de mercado a saltos Markovianos. Bauerle e Rieder (2004) investigaram, para diferentes funções utilidades da riqueza final, um modelo de otimização em tempo contínuo com dois ativos e parâmetros sujeitos a saltos Markovianos. Utilizando o enfoque de média-variância, uma evolução do modelo de Zhou e Li (2000) para o caso em que os parâmetros estão sujeitos a saltos Markovianos foi realizada em (Zhou e Yin (2003)). Estes últimos autores, em (Yin e Zhou (2004)), também investigaram uma versão em tempo discreto deste último modelo. Entretanto, é importante enfatizar que Yin e Zhou (2004) não derivaram analiticamente em tempo discreto uma fórmula fechada para a solução ótima do problema de média-variância multi-período com saltos. A idéia básica em (Yin e Zhou (2004)) é a de aproveitar a estratégia ótima do problema em tempo contínuo, resolvido em (Zhou e Yin (2003)), como solução sub-ótima, ou seja, apenas assintóticamente ótima, para o problema em tempo discreto, mostrando que, convenientemente interpolados, os processos em tempo discreto convergem de forma fraca para seus limites a tempo contínuo. (Çakmak e Özekici (2006)) derivaram analiticamente uma solução ótima para o problema tradicional em multi-período de médiavariância com saltos Markovianos (formulação I). Portanto, em (Çakmak e Özekici (2006)) evitou-se qualquer premissa de aproximação como requeridas no modelo de Yin e Zhou (2004), e os resultados obtidos são ótimos, em vez de apenas assintóticamente ótimos como em (Yin e Zhou (2004)).

Entretanto, Çakmak e Özekici (2006) investigaram apenas o caso em que o mercado financeiro é formado por $n$ ativos de risco mais um ativo livre de risco, que também depende da cadeia de Markov. Portanto, os resultados obtidos em (Çakmak e Özekici (2006)) são generalizados nesta tese para o caso em que todos os ativos do universo de 
investimento são de risco e para o caso em que existe um ativo livre de risco, que não sofre influência de qualquer fator de incerteza.

Além disso, tanto Çakmak e Özekici (2006) como Yin e Zhou (2004) consideraram apenas o caso particular no qual não se leva em conta os períodos intermediários na função objetivo e nas restrições. Dessa forma, apesar de considerarem uma abordagem em multi-período, a formulação dos modelos de média-variância em Çakmak e Özekici (2006) e em Yin e Zhou (2004) são iguais às originais descritas em (2.1) e (2.2).

Em (Zhu et al. (2004)), os autores se utilizaram da inequação de Tchebycheff para propor um modelo multi-período de média-variância com restrições sobre a probabilidade de falência do portfólio nos períodos intermediários. O modelo de Zhu et al. (2004) possui a seguinte formulação:

$$
\begin{aligned}
& \max E(V(T)) \\
& \text { sujeito a: } \operatorname{Var}(V(t)) \leq \varrho(t)[E(V(t))-\psi(t)]^{2}, t=1, \ldots, T,
\end{aligned}
$$

onde $\varrho(t)$ representa a probabilidade máxima do portfólio cair abaixo de um determinado valor mínimo, definido por $\psi(t)$. No entanto, neste modelo, apenas as restrições incidem sobre os períodos intermediários e os coeficientes de mercado ( $\mu$ e $\sigma)$ na modelagem dos preços são determinísticos.

Ressalta-se novamente que os trabalhos até o momento apenas derivaram condições necessárias para as soluções ótimas de seus modelos. Portanto, desconhece-se a existência de trabalhos que derivaram condições suficientes para a obtenção da solução ótima a tempo discreto de problemas multi-período de seleção de carteira em média-variância com saltos Markovianos.

Outra abordagem relevante no processo de seleção de carteiras é aquela em que o objetivo é obter um desempenho superior a um índice de mercado. Como descrito por diversos autores, entre eles Roll (1992), Brennan (1993), Chow (1995), Browne (1999), Costa e Paiva (2002), Ammann e Zimmermann (2001), Jorion (2003), Fabozzi et al. (2004) e Alexander e Baptista (2005), os gestores de carteiras estão usualmente mais 
preocupados com o desempenho de suas carteiras em relação a um determinado benchmark do que com seu retorno absoluto.

Este enfoque foi inicialmente formalizado por Roll (1992), que modelou o problema de média-variância do tracking error em período simples e com coeficientes determinísticos. A formulação de Roll (1992) é similar aos modelos (2.1) e (2.2), apenas substituindo a variável patrimônio final do investidor $(V(T))$ por seu tracking error $(G(T))$ nas expressões da esperança e da variância, conforme abaixo:

$$
\begin{gathered}
\max E(G(T)) \\
\text { sujeito a }: \quad \operatorname{Var}(G(T)) \leq \sigma^{2}
\end{gathered}
$$

e

$$
\begin{array}{ll} 
& \min \operatorname{Var}(G(T)) \\
\text { sujeito a }: & E(G(T)) \geq \epsilon .
\end{array}
$$

Apesar do relacionamento direto entre as duas abordagens, desconhece-se que resultados em tempo discreto para o problema multi-período de média-variância do tracking error com parâmetros sujeitos a saltos Markovianos tenham sido obtidos anteriormente. 


\section{Capítulo 3}

\section{Descrição do Mercado e Formulação}

\section{dos Problemas}

Neste capítulo, apresenta-se uma descrição do mercado a ser investigado, definindo os ativos que o formam e o processo que rege a evolução dos preços destes ativos. Além disso, também são formulados os problemas de média-variância que serão investigados pelo resto do trabalho.

Neste trabalho a seguinte notação será adotada. Denota-se por $\mathbb{R}^{n}$ o espaço Euclideano $n$-dimensional e por $\mathbb{R}^{n \times m}$ o espaço Euclideano de todas as $n \times m$ matrizes reais. Para uma sequência de números $a_{1}, \ldots, a_{m}$, define-se como diag $\left(a_{i}\right)$ a matriz diagonal em $\mathbb{R}^{m \times m}$ composta pelos elementos $a_{i}$ nas $i$-ésimas posições da diagonal, com $i=1, \ldots, m$. O superscrito ' indicará o transposto de um vetor ou matriz.

\subsection{Descrição do Mercado}

No modelo em análise, considera-se um mercado formado por $n+1$ ativos em um espaço de probabilidade completo $\left(\Omega, \mathcal{F},\left\{\mathcal{F}_{t}\right\}, \mathcal{P}\right)$. A cadeia finita de Markov em tempo 
discreto $\{\theta(t) ; t=0, \ldots, T\}$, tomando valores no conjunto $\mathcal{M}=\{1, \ldots, m\}$, representa os possíveis estados da economia ou modos do mercado, que regem a tendência geral de movimento dos ativos. $\mathcal{P}$ é uma medida de probabilidade tal que:

$$
\mathcal{P}(\theta(t+1)=j \mid \theta(0), \ldots, \theta(t)=i)=\mathcal{P}(\theta(t+1)=j \mid \theta(t)=i)=p_{i j}(t)
$$

$\operatorname{com} t=0, \ldots, T-1, p_{i j}(t) \geq 0$ e $\sum_{j} p_{i j}(t)=1$, para $i, j \in \mathcal{M}$. Para $t=0, \ldots, T$, estabelece-se

$$
\begin{aligned}
P(t) & =\left[p_{i j}(t)\right]_{m \times m}, \\
\pi(t) & =\left(\pi_{1}(t), \ldots, \pi_{m}(t)\right)^{\prime} \\
\pi_{i}(t) & =\mathcal{P}(\theta(t)=i) .
\end{aligned}
$$

Como em Costa et al. (2005), para $z=\left(z_{1}, \ldots, z_{m}\right)^{\prime} \in \mathbb{R}^{m}$, determina-se o operador $\mathcal{E}(z, t)=\left(\mathcal{E}_{1}(z, t), \ldots, \mathcal{E}_{m}(z, t)\right)$ como:

$$
\mathcal{E}_{i}(z, t)=\sum_{j=1}^{m} p_{i j}(t) z_{j}, \text { para } i \in \mathcal{M}
$$

Por simplicidade notacional, omitir-se-á, pelo restante do texto, a variável $t$ em $\mathcal{E}(z, t)$, indicando-a como $\mathcal{E}(z)$, onde não houver dúvida quanto à sua colocação.

O preço dos títulos é descrito pelo vetor aleatório $\overline{\mathrm{S}}(t)=\left(\mathrm{S}_{0}(t), \ldots, \mathrm{S}_{n}(t)\right)^{\prime} \in \mathbb{R}^{n+1}$ com $t=0, \ldots, T$. A filtração $\mathcal{F}_{t}$ é tal que os vetores aleatórios $\{\overline{\mathrm{S}}(k) ; k=0, \ldots, t\}$ e a cadeia de Markov $\{\theta(k) ; k=0, \ldots, t\}$ são $\mathcal{F}_{t^{-}}$-mensuráveis.

Quando o modo de operação do mercado é $\theta(t)=i \in \mathcal{M}, \bar{\mu}(t, i) \in \mathbb{R}^{n+1}$ representa o vetor com a expectativa de retorno dos ativos e $\bar{\sigma}(t, i) \bar{\sigma}(t, i)^{\prime} \in \mathbb{R}^{(n+1) \times(n+1)}$ é a matriz de covariância dos retornos dos ativos. Por conveniência, decompõe-se $\bar{\mu}(t, i)$ e $\bar{\sigma}(t, i)$ como:

$$
\bar{\mu}(t, i)=\left[\begin{array}{l}
\mu_{0}(t, i) \\
\mu(t, i)
\end{array}\right]
$$


$\mathrm{e}$

$$
\bar{\sigma}(t, i)=\left[\begin{array}{c}
\sigma_{0}(t, i) \\
\sigma(t, i)
\end{array}\right]
$$

com

$$
\begin{gathered}
\mu(t, i)=\left(\mu_{1}(t, i), \ldots, \mu_{n}(t, i)\right)^{\prime} \in \mathbb{R}^{n}, \\
\sigma_{0}(t, i)=\left(\sigma_{00}(t, i), \ldots, \sigma_{0 n}(t, i)\right) \in \mathbb{R}^{1 \times n+1}
\end{gathered}
$$

$\mathrm{e}$

$$
\sigma(t, i)=\left[\begin{array}{ccc}
\sigma_{10}(t, i) & \cdots & \sigma_{1 n}(t, i) \\
\vdots & \ddots & \vdots \\
\sigma_{n 0}(t, i) & \cdots & \sigma_{n n}(t, i)
\end{array}\right] \in \mathbb{R}^{n \times n+1} .
$$

Observa-se que o retorno esperado de cada ativo e a covariância destes dependem da cadeia de Markov, ou seja, dependem por exemplo do estado da economia ou do humor geral dos investidores. Portanto, destaca-se, que a incerteza com relação a estes estados influencia diretamente estes dois parâmetros chave na definição da rentabilidade dos ativos a ser descrita a seguir.

Seja a rentabilidade dos preços dos ativos definida como o vetor aleatório $\overline{\mathrm{R}}(t)=$ $\left(\mathrm{R}_{0}(t), \ldots, \mathrm{R}_{n}(t)\right)^{\prime}, \mathrm{com}$

$$
\mathrm{R}_{j}(t)=\frac{\mathrm{S}_{j}(t+1)}{\mathrm{S}_{j}(t)}
$$

satisfazendo o seguinte processo:

$$
\overline{\mathrm{R}}_{\theta(t)}(t)=[\bar{e}+\bar{\mu}(t, \theta(t))]+\bar{\sigma}(t, \theta(t)) W(t),
$$

onde

$$
\bar{e}=\left(1, e^{\prime}\right)^{\prime},
$$

com

$$
e=(1, \ldots, 1)^{\prime}
$$

um vetor de dimensão conveniente com $1^{\prime} s$ em todos os seus componentes e $\{W(t) ; t=$ 
$0, \ldots, T\}$ é uma sequência de vetores aleatórios independentes de dimensão $(n+1)$ com média zero e covariância $I$ (matriz identidade). Considera-se que $\{W(t), \theta(t)\}$ são mutuamente independentes. Assim, tem-se que $E[W(t)]=0$ e $E\left[W(t) W(t)^{\prime}\right]=I$.

Ressalta-se, portanto, a influência das duas fontes de incerteza sobre a rentabilidade dos ativos: a cadeia de Markov representada por $\theta(t)$ e o vetor randômico representado por $W(t)$.

Assume-se que:

$$
E\left(\overline{\mathrm{R}}(t) \overline{\mathrm{R}}(t)^{\prime} \mid \theta(t)=i\right)>0
$$

para cada $t=0, \ldots, T-1$ e $i \in \mathcal{M}$.

\subsection{O Portfólio}

Seja o vetor $\bar{u}(t)=\left(u_{0}(t), u(t)\right)^{\prime} \in \mathbb{R}^{n+1}$, onde $u(t)=\left(u_{1}(t), \ldots, u_{n}(t)\right)^{\prime}$ é definido como o vetor que representa o montante da riqueza alocado nos ativos $j=1$ a $j=n$, e $u_{0}(t)$ o montante do patrimônio investido no ativo $j=0$.

Definição $3.1 O$ conjunto de estratégias admissiveis de investimento $\mathcal{U}=\{u=$ $(\bar{u}(0), \ldots, \bar{u}(T-1))\}$ é tal que para cada $t=0, \ldots, T-1, \bar{u}(t)=\left(u_{0}(t), \ldots, u_{n}(t)\right)^{\prime}$ é um vetor aleatório $\mathcal{F}_{t}$ mensurável, tomando valores em $\mathbb{R}^{n+1}$.

Associado a cada estratégia admissível de investimento $u$, determina-se o processo do valor de um portfólio como $\left\{V^{u}(t) \in \mathbb{R} ; t=0, \ldots, T\right\}$, que representa a riqueza do investidor ao final do tempo t. Por simplicidade notacional, o superscrito ${ }^{u}$ será suprimido onde não se levante dúvida. Dessa forma, o patrimônio do investidor no instante $t$ é expresso por:

$$
V(t)=\bar{u}(t)^{\prime} \bar{e}=u_{0}(t)+u(t)^{\prime} e .
$$


Observa-se que o montante da riqueza alocado no ativo $i=0\left(u_{0}(t)\right)$ é completamente determinado por $V(t)-e^{\prime} u(t)$. Esta igualdade será utilizada mais tarde para excluir $u_{0}(t)$ do problema de otimização.

Determinando como $V(0)=V_{0}>0$ o patrimônio inicial do investidor e que o portfólio é auto-financiado, ou seja, não há captações ou resgates de recursos na carteira de investimentos, a riqueza do investidor evolui, como por exemplo em Li e Ng (2000), conforme a equação:

$$
\begin{aligned}
V(t+1) & =\bar{u}(t)^{\prime} \overline{\mathrm{R}}_{\theta(t)}(t) \\
& =u_{0}(t)\left(1+\mu_{0}(t, \theta(t))+\sigma_{0}(t, \theta(t)) W(t)\right) \\
& +u(t)^{\prime}(e+\mu(t, \theta(t))+\sigma(t, \theta(t)) W(t)) .
\end{aligned}
$$

Substituindo $u_{0}$, determinado por (3.7), na equação acima, tem-se que:

$$
\begin{aligned}
V(t+1)= & \left(V(t)-u(t)^{\prime} e\right)\left(1+\mu_{0}(t, \theta(t))+\sigma_{0}(t, \theta(t)) W(t)\right) \\
& +u(t)^{\prime}(e+\mu(t, \theta(t))+\sigma(t, \theta(t)) W(t)) \\
= & V(t)\left(1+\mu_{0}(t, \theta(t))+\sigma_{0}(t, \theta(t)) W(t)\right) \\
& -u(t)^{\prime}\left(e+e \mu_{0}(t, \theta(t))+e \sigma_{0}(t, \theta(t)) W(t)\right) \\
& +u(t)^{\prime}(e+\mu(t, \theta(t))+\sigma(t, \theta(t)) W(t)) \\
= & V(t)\left(1+\mu_{0}(t, \theta(t))+\sigma_{0}(t, \theta(t)) W(t)\right) \\
& +u(t)^{\prime}\left(\mu(t, \theta(t))-e \mu_{0}(t, \theta(t))\right. \\
& \left.+\left(\sigma(t, \theta(t))-e \sigma_{0}(t, \theta(t))\right) W(t)\right) .
\end{aligned}
$$


Definindo:

$$
\begin{aligned}
& \hat{A}_{\theta(t)}(t)=\left(1+\mu_{0}\left(t, \theta_{t}\right)\right), \\
& \tilde{A}_{\theta(t)}(t)=\left[\sigma_{0}(t, \theta(t))\right], \\
& \hat{B}_{\theta(t)}(t)=\left[\mu(t, \theta(t))-e \mu_{0}(t, \theta(t))\right], \\
& \tilde{B}_{\theta(t)}(t)=\left[\sigma(t, \theta(t))-e \sigma_{0}(t, \theta(t))\right],
\end{aligned}
$$

reescreve-se (3.9) como:

$$
V(t+1)=A_{\theta(t)}(t) V(t)+B_{\theta(t)}(t)^{\prime} u(t)
$$

onde

$$
\begin{aligned}
& A_{\theta(t)}(t)=\hat{A}_{\theta(t)}(t)+\widetilde{A}_{\theta(t)}(t) W(t), \\
& B_{\theta(t)}(t)=\hat{B}_{\theta(t)}(t)+\widetilde{B}_{\theta(t)}(t) W(t) .
\end{aligned}
$$

A seguir, apresentam-se algumas definições que serão necessárias pelo resto do texto. Para cada $i, j \in \mathcal{M}$ e $t \in \mathcal{T}$, define-se:

$$
\begin{aligned}
\chi_{i}(t) & =E\left(B_{i}(t)\right)=\hat{B}_{i}(t), \\
\phi_{i}(t) & =E\left(B_{i}(t) B_{i}(t)^{\prime}\right)=\hat{B}_{i}(t) \hat{B}_{i}(t)^{\prime}+\widetilde{B}_{i}(t) \widetilde{B}_{i}(t)^{\prime}, \\
\delta_{i}(t) & =E\left(A_{i}(t)^{2}\right)=\hat{A}_{i}(t)^{2}+\left\|\tilde{A}_{i}(t)\right\|^{2}, \\
\varphi_{i}(t)^{\prime} & =E\left(A_{i}(t) B_{i}(t)^{\prime}\right)=\hat{A}_{i}(t) \hat{B}_{i}(t)^{\prime}+\tilde{A}_{i}(t) \tilde{B}_{i}(t)^{\prime}, \\
\beta_{i}(t) & =\chi_{i}(t)^{\prime} \phi_{i}(t)^{-1} \chi_{i}(t),
\end{aligned}
$$




$$
\begin{aligned}
\beta(t) & =\operatorname{diag}\left(\beta_{i}(t)\right) \\
Q_{i}(t) & =\delta_{i}(t)-\varphi_{i}(t)^{\prime} \phi_{i}(t)^{-1} \varphi_{i}(t), \\
Q(t) & =\operatorname{diag}\left(Q_{i}(t)\right), \\
R_{i}(t) & =\hat{A}_{i}(t)-\chi_{i}(t)^{\prime} \phi_{i}(t)^{-1} \varphi_{i}(t), \\
R(t) & =\operatorname{diag}\left(R_{i}(t)\right) .
\end{aligned}
$$

A partir da condição (3.6), a inversa de $\phi_{i}(t)$ é bem definida. Isto pode ser justificado pela expressão:

$$
\begin{gathered}
\left(\begin{array}{cc}
E\left(A_{i}(t)^{2}\right) & E\left(A_{i}(t) B_{i}(t)^{\prime}\right) \\
E\left(A_{i}(t) B_{i}(t)\right) & E\left(B_{i}(t) B_{i}(t)^{\prime}\right)
\end{array}\right)= \\
\left(\begin{array}{cc}
1 & 0 \\
-e & I
\end{array}\right) E\left(\overline{\mathrm{R}}_{\theta(t)}(t) \overline{\mathrm{R}}_{\theta(t)}(t)^{\prime}\right)\left(\begin{array}{cc}
1 & -e^{\prime} \\
0 & I
\end{array}\right)>0
\end{gathered}
$$

para todo $t=0, \ldots, T-1$ e $i \in \mathcal{M}$. Portanto, $\phi_{i}(t)$ é positivo definido e $Q_{i}(t)>0$ e $R_{i}(t)>0$ para todos os períodos.

\subsection{Formulação do Problema Generalizado de Média-Variância e dos Modelos Derivados}

Os problemas tradicionais de média-variância em multi-período buscam selecionar um conjunto de portfólios que proporcionem a maior riqueza final esperada para um nível de risco desejado para o instante final (variância da riqueza final), ou encontrar uma sequência de portfólios que produzam a menor variância do patrimônio terminal do investidor para um desejado valor esperado deste patrimônio final.

Formalmente, as duas formulações citadas acima, nomeadas respectivamente de 
$P\left(\sigma^{2}\right)$ e $P(\epsilon)$, podem ser expressas como:

$$
\begin{aligned}
P\left(\sigma^{2}\right) & : \max _{u \in \mathcal{U}} E(V(T)) \\
\text { sujeito a } & : \operatorname{Var}(V(T)) \leq \sigma^{2}
\end{aligned}
$$

e

$$
\begin{aligned}
P(\epsilon) & : \max _{u \in \mathcal{U}}-\operatorname{Var}(V(T)) \\
\text { sujeito a } & : \quad E(V(T)) \geq \epsilon .
\end{aligned}
$$

Como já comentado, problemas como estes foram resolvidos analiticamente em tempo discreto e contínuo para o caso sem saltos Markovianos em Li e Ng (2000) e Zhou e Li (2000), respectivamente, e em Çakmak e Özekici (2006), quando foi incluída a possibilidade de saltos nas variáveis de mercado (retorno esperado dos ativos e variância dos retornos).

No entanto, estas expressões não consideram os valores da riqueza nem do risco nos períodos intermediários. Algumas classes de investidores, como as entidades de previdência e de seguros, devem manter controles e, regularmente (anualmente ou trimestralmente), prestar contas a órgãos reguladores sobre pontos definidos em suas políticas de investimentos como o retorno, o risco e o nível de seus patrimônios que devem honrar seus passivos. Portanto, estes investidores necessitam acompanhar ao longo de todo o período de investimento a evolução do patrimônio e do risco incorrido em cada instante. Dessa forma, estes investidores devem incluir os períodos intermediários em suas funções objetivo e restrições.

Define-se $\mathcal{T}:=\left\{\tau_{1}, \ldots, \tau_{\iota_{f}}\right\}$, com $\tau_{0}=0$ e $\tau_{\iota_{f}}=T$. Introduz-se o coeficiente $\alpha(t) \geq 0$, para todo $t \in \mathcal{T}$, com $\alpha(T)>0$ e $\alpha(t)=0$, para $t \notin \mathcal{T}$, como um conjunto de números reais positivos que representará o peso que o investidor atribui à riqueza (ou risco) a cada período desejado de seu horizonte de investimento.

Para atender à demanda daqueles investidores institucionais, uma formulação mais 
apropriada é a que a função objetivo é constituída pela soma ponderada do valor esperado da riqueza ao longo do tempo, tendo como restrição o risco (variância do patrimônio) em cada instante $t \in \mathcal{T}$. Outra formulação indicada a estes investidores é a que a função objetivo é minimizar a soma ponderada da variância da riqueza ao longo do tempo, tendo como restrição a esperança do patrimônio em cada instante $t \in \mathcal{T}$. Portanto, reformulam-se os problemas $P\left(\sigma^{2}\right)$ e $P(\epsilon)$, denominando-os, respectivamente de $P M V\left(\sigma^{2}\right)$ e $P M V(\epsilon)$, como:

$$
\begin{aligned}
\operatorname{PMV}\left(\sigma^{2}\right) & : \max _{u \in \mathcal{U}} \sum_{t \in \mathcal{T}} \alpha(t) E(V(t)) \\
\text { sujeito a } & : \quad \operatorname{Var}(V(t)) \leq \sigma^{2}(t), t \in \mathcal{T}
\end{aligned}
$$

$\mathrm{e}$

$$
\begin{array}{ll}
P M V(\epsilon) & : \max _{u \in \mathcal{U}}-\sum_{t \in \mathcal{T}} \alpha(t) \operatorname{Var}(V(t)) \\
\text { sujeito a } & : \quad E(V(t)) \geq \epsilon(t), t \in \mathcal{T} .
\end{array}
$$

A relevância que o investidor atribui a cada instante de tempo $t \in \mathcal{T}$ pode ser controlada através do parâmetro $\alpha(t)$. Quanto maior for o valor atribuído a este coeficiente em um determinado instante em relação aos outros valores de $\alpha(t)$, maior será a importância da esperança ou da variância da riqueza neste período em relação aos outros instantes de tempo no processo de otimização.

Entretanto, estas formulações ainda desconsideram a possibilidade do portfólio falir antes do período final, ou seja, uma possível perda completa do patrimônio em um instante intermediário. Como demonstrado por Zhu et al. (2004), na formulação multiperíodo tradicional de média-variância o portfólio pode ter seu patrimônio reduzido a um valor negativo e mesmo assim continuar investindo em ativos através da captação de recursos oriundos da venda a descoberto de outros ativos. No entanto, a possibilidade de um investidor com patrimônio negativo continuar operando no mercado é praticamente inviável em casos reais. De fato, muitos investidores, como entidades de 
previdência, possuem metas de rentabilidade mínima ou metas atuariais e não podem permitir que seus patrimônios caiam abaixo de um limite mínimo sob pena de sofrerem sanções de órgãos reguladores e sob pressão das empresas patrocinadoras que podem ter de elevar o nível de contribuição para que os ativos sejam suficientes para fazer frente aos passivos.

Zhu et al. (2004) apresentaram uma estratégia para controlar a probabilidade de perda máxima ou de falência de portfólios. Este controle pode ser realizado através da inequação de Tchebycheff. Dessa forma, assumindo um nível mínimo aceitável para o valor do portfólio em cada período $t$ como $V(t)_{\text {minímo }}=\psi(t)$, tem-se que a probabilidade do patrimônio do investidor em cada instante $t$ ser menor que este valor mínimo pode ser limitada pela inequação:

$$
P(V(t) \leq \psi(t)) \leq \frac{\operatorname{Var}(V(t))}{[E(V(t))-\psi(t)]^{2}} \leq \varrho(t)
$$

onde $\varrho(t)$ é uma variável informada pelo investidor, representando seu desejo com relação à probabilidade máxima de seu patrimônio cair abaixo do valor mínimo $\psi(t)$, também definido por este mesmo agente.

Portanto, inserindo a restrição de controle sobre a probabilidade máxima da riqueza ser inferior a um determinado limite mínimo, mas sem considerar a possibilidade de saltos Markovianos nos parâmetros de mercado, Zhu et al. (2004) reescreveram o problema $P\left(\sigma^{2}\right)$ como:

$$
\begin{aligned}
& \operatorname{PCF}(\varrho, \psi) \quad: \max _{u \in \mathcal{U}} E(V(T)) \\
& \operatorname{Var}(V(t)) \leq \varrho(t)[E(V(t))-\psi(t)]^{2}, t=1, \ldots, T
\end{aligned}
$$

onde $\varrho=\left(\varrho\left(\tau_{1}\right), \varrho\left(\tau_{2}\right), \ldots, \varrho\left(\tau_{\iota_{f}}\right)\right)^{\prime}$ e $\psi=\left(\psi\left(\tau_{1}\right), \psi\left(\tau_{2}\right), \ldots, \psi\left(\tau_{\iota_{f}}\right)\right)^{\prime}$ são vetores, cujos componentes $\varrho(t)$ e $\psi(t)$ indicam a probabilidade máxima do portfólio cair abaixo de um determinado valor mínimo ao final do período $t$, e este valor mínimo, respectivamente. 
Novamente, ressalta-se a vantagem de permitir que o investidor possa ponderar da forma que achar mais adequado a relevância do valor esperado da riqueza em cada instante no processo de otimização. Dessa forma, inclui-se ao problema $P C F(\varrho, \psi)$ a possibilidade de ponderação da riqueza ao longo do tempo e a existência de saltos Markovianos nas variáveis de mercado, reescrevendo-o como:

$$
\begin{aligned}
& P B C(\varrho, \psi) \quad: \max _{u \in \mathcal{U}} \sum_{t \in \mathcal{T}} \alpha(t) E(V(t)) \\
& \operatorname{Var}(V(t)) \leq \varrho(t)[E(V(t))-\psi(t)]^{2}, t \in \mathcal{T} .
\end{aligned}
$$

Destaca-se que o problema $P B C(\varrho, \psi)$ engloba o problema $P M V\left(\sigma^{2}\right)$, já que a restrição sobre a variância final em (3.29) pode ser replicada em (3.31), bastando escolher convenientemente as variáveis $\varrho(T)$ e $\psi(T)$.

\subsubsection{Formulação para o Problema Generalizado de Média-Variância com Saltos Markovianos}

As formulações citadas acima e outras variações dos problemas de média-variância são derivadas de uma forma generalizada do problema multi-período de média-variância, que será descrita a seguir e que será alvo de estudo neste trabalho.

Como descrito anteriormente, o problema multi-período generalizado de médiavariância com saltos Markovianos $(P G M V)$ é um problema de controle estocástico sem restrição cuja função objetivo consiste em maximizar a soma ponderada ao longo do tempo da combinação linear de três elementos: o valor esperado da riqueza do investidor, o quadrado da esperança desta riqueza e a esperança do quadrado deste patrimônio.

Considere uma sequência de números positivos $\rho(t), \nu(t)$, e uma sequência de números reais $\ell(t)$, para $t \in \mathcal{I}:=\left\{\tau_{1}, \ldots, \tau_{\iota_{f}}\right\}$, com $\rho(T)>0, \nu(T)>0$, e $\ell(T) \neq 0$. 
Define-se, portanto o problema o PGMV como:

$$
P G M V(\rho, \ell, \nu): \max _{u \in \mathcal{U}} \sum_{t \in \mathcal{I}}\left(\nu(t) E(V(t))^{2}-\rho(t) E\left(V(t)^{2}\right)+\ell(t) E(V(t))\right) .
$$

Convenientemente, estende-se a definição de $\ell(t), \nu(t)$ e $\rho(t)$ para $t \notin \mathcal{I}$, fixando nestes casos $\ell(t)=0, \nu(t)=0$ e $\rho(t)=0$. Além disso, define-se para o momento inicial $\tau_{0}=0, \ell(0)=0, \nu(0)=0$ e $\rho(0)=0$.

As formulações $P\left(\sigma^{2}\right), P(\epsilon), P M V\left(\sigma^{2}\right), P M V(\epsilon)$ e $P B C(\varrho, \psi)$ podem ser reescritas na formulação sem restrição $\operatorname{PGMV}(\rho, \ell, \nu)$, através da introdução de Multiplicadores de Lagrange. Este processo será apresentado a seguir.

Iniciando com o problema $P B C(\varrho, \psi)$, insere-se os Multiplicadores de Lagrange não negativos $\omega(t)$, com $t \in \mathcal{I}=\mathcal{T}$ e $\omega(T) \neq 0$ em (3.31). Dessa forma, reescreve-se PBC $(\varrho, \psi)$ como um problema de maximização Lagrangiano $(L P B C(\omega, \varrho, \psi))$ descrito por:

$$
\begin{aligned}
L P B C(\omega, \varrho, \psi): \max _{u \in \mathcal{U}} \sum_{t \in \mathcal{T}} \alpha(t) E(V(t)) & \\
& -\sum_{t \in \mathcal{T}} \omega(t)\left(\operatorname{Var}(V(t))-\varrho(t)[E(V(t))-\psi(t)]^{2}\right),
\end{aligned}
$$

Expandindo o termo $\operatorname{Var}(V(k))$ como $\operatorname{Var}(V(k))=E\left(V(k)^{2}\right)-E(V(k))^{2}$, e desenvolvendo o termo ao quadrado no lado direito da equação, determina-se:

$$
\begin{aligned}
& \operatorname{LPBC}(\omega, \varrho, \psi): \max _{u \in \mathcal{U}} \sum_{t \in \mathcal{T}}(\alpha(t)-2 \omega(t) \varrho(t) \psi(t)) E(V(t))-\sum_{t \in \mathcal{T}} \omega(t) E\left(V(t)^{2}\right) \\
& +\sum_{t \in \mathcal{T}} \omega(t)(1+\varrho(t)) E(V(t))^{2}+\sum_{t \in \mathcal{T}} \omega(t) \varrho(t) \psi(t)^{2} .
\end{aligned}
$$


Estabelece-se uma relação entre $\operatorname{LPBC}(\omega, \varrho, \psi)$ e $P G M V(\rho, \ell, \nu)$, fazendo, para $t \in \mathcal{I}$,

$$
\left\{\begin{array}{l}
\rho(t)=\omega(t) \\
\nu(t)=\rho(t)(1+\varrho(t)) \\
\ell(t)=\alpha(t)-2 \rho(t) \varrho(t) \psi(t) .
\end{array}\right.
$$

A relação entre $P M V\left(\sigma^{2}\right)$ e $P M V(\epsilon)$ para o problema $P G M V(\rho, \ell, \nu)$ é bem mais direta. Seguindo o mesmo procedimento, insere-se os Multiplicadores de Lagrange não negativos $\omega(t)$, com $t \in \mathcal{I}$, definindo o problema sem restrição $\left(\operatorname{LP} M V\left(\sigma^{2}\right)\right)$ :

$$
\begin{aligned}
\operatorname{LPMV}\left(\omega, \sigma^{2}\right): & \max _{u \in \mathcal{U}} \sum_{t \in \mathcal{T}} \alpha(t) E(V(t))-\sum_{t \in \mathcal{T}} \omega(t) \operatorname{Var}(V(t)) \\
: & \max _{u \in \mathcal{U}} \sum_{t \in \mathcal{T}} \alpha(t) E(V(t))-\sum_{t \in \mathcal{T}} \omega(t)\left(E\left(V(k)^{2}\right)-E(V(k))^{2}\right) \\
: & \max _{u \in \mathcal{U}} \sum_{t \in \mathcal{T}}\left(\omega(t)\left(E(V(k))^{2}-E\left(V(k)^{2}\right)\right)+\alpha(t) E(V(t))\right) .
\end{aligned}
$$

Neste caso, a relação entre $\operatorname{LPMV}\left(\omega, \sigma^{2}\right)$ e $\operatorname{PGMV}(\rho, \ell, \nu)$ é obtida fazendo, para $t \in \mathcal{I}$

$$
\left\{\begin{array}{l}
\rho(t)=\nu(t)=\omega(t) \\
\ell(t)=\alpha(t)
\end{array}\right.
$$

Para o problema $\operatorname{PMV}(\epsilon)$, a relação segue diretamente do processo descrito para $P M V\left(\sigma^{2}\right)$. Dessa forma, define-se o problema sem restrição $(\operatorname{LP} M V(\omega, \epsilon))$ :

$$
\begin{aligned}
\operatorname{LPMV}(\omega, \epsilon) & : \max _{u \in \mathcal{U}}-\sum_{t \in \mathcal{T}} \alpha(t) \operatorname{Var}(V(t))-\sum_{t \in \mathcal{T}} \omega(t) E(V(t)) \\
& : \max _{u \in \mathcal{U}}-\sum_{t \in \mathcal{T}} \alpha(t)\left(E\left(V(k)^{2}\right)-E(V(k))^{2}\right)-\sum_{t \in \mathcal{T}} \omega(t) E(V(t)) \\
& : \max _{u \in \mathcal{U}} \sum_{t \in \mathcal{T}}\left(\alpha(t)\left(E(V(k))^{2}-E\left(V(k)^{2}\right)\right)-\omega(t) E(V(t))\right),(3.37)
\end{aligned}
$$


e a relação entre $L P M V(\omega, \epsilon)$ e $P G M V(\rho, \ell, \nu)$ é obtida fazendo, para $t \in \mathcal{I}$,

$$
\left\{\begin{array}{l}
\rho(t)=\nu(t)=\alpha(t) \\
\ell(t)=-\omega(t)
\end{array}\right.
$$

Os problemas $P\left(\sigma^{2}\right)$ e $P(\epsilon)$ têm uma formulação similar a $P M V\left(\sigma^{2}\right)$ e $P M V(\epsilon)$, diferenciando-se apenas por suas funções objetivo e restrições atuarem apenas no instante final $(T)$. Portanto, para $P\left(\sigma^{2}\right)$ e $P(\epsilon)$, nota-se que $\omega(t)=0, \alpha(t)=0$, para $t \neq T$, e $\alpha(T)=1$ e $\omega(T)>0$, em $(3.36)$ e (3.38), respectivamente.

\subsubsection{Formulação do Problema Auxiliar}

Como descrito em Li e Ng (2000) o problema estocástico multi-período de médiavariância apresenta a característica de ser não separável no sentido da programação dinâmica. Isto ocorre porque o problema envolve uma função utilidade não linear de uma esperança (mais especificamente o quadrado da esperança da riqueza $\left(E(V(t))^{2}\right)$ ), e, portanto, não pode ser diretamente resolvido através da programação dinâmica. Li e Ng (2000) propuseram uma solução para esta impossibilidade de se resolver problemas como o $\operatorname{PGMV}(\rho, \ell, \nu)$, através da programação dinâmica. Esta solução é baseada em um problema auxiliar que pode ser resolvido através da programação dinâmica. Este mesmo procedimento foi utilizado por Zhou e Li (2000), Zhou e Yin (2003), Yin e Zhou (2004), Zhu et al. (2004) e Çakmak e Özekici (2006).

Dessa forma, para a solução do problema $P G M V(\rho, \ell, \nu)$, considerou-se uma formulação auxiliar como a desenvolvida em Li e Ng (2000). Seja $\lambda(t)$ um conjunto de números reais, para $t=1, \ldots, T$, atribui-se a $P G M V(\rho, \ell, \nu)$ o seguinte problema auxiliar:

$$
A(\lambda, \rho): \min _{u \in \mathcal{U}} \sum_{t=1}^{T} E\left\{\rho(t) V(t)^{2}-\lambda(t) V(t)\right\} .
$$

Também se estende a definição de $\lambda(t)$ para $t \notin \mathcal{I}$, como $\lambda(t)=0$. 
A formulação auxiliar $A(\lambda, \rho)$ tem a vantagem de possuir uma função objetivo quadrática separável no sentido da programação dinâmica. 


\section{Capítulo 4}

\section{Solução para o Problema Generalizado}

\section{de Média-Variância com Parâmetros}

\section{Sujeitos a Saltos Markovianos}

Neste capítulo investiga-se o problema generalizado de média-variância $(P G M V(\rho, \ell, \nu))$ descrito na Seção 3.3 para o caso geral em que o mercado é formado apenas por ativos de risco. Inicialmente, deriva-se analiticamente, através da programação dinâmica, uma lei de controle ótima para o problema auxiliar $A(\lambda, \rho)$ descrito no capítulo anterior e se obtém uma expressão para a função valor deste problema. A partir desta estratégia ótima de controle, determinam-se expressões explícitas para a solução do problema generalizado de média-variância com parâmetros sujeitos a saltos Markovianos.

\subsection{Lei de Controle Ótima}

A função custo associada ao problema auxiliar (3.39) com uma política ótima de controle admissível $u=(u(0), \ldots, u(T-1))$ e com condições iniciais $(V(0), \theta(0))$ é 
denotada por $J(V(0), \theta(0))$, e seu mínimo por $J^{*}(V(0), \theta(0))$. Assim, objetiva-se encontrar uma política admissível de controle $u \in \mathcal{U}$ tal que:

$$
J^{*}(V(0), \theta(0))=\min _{u \in \mathcal{U}} \sum_{t=1}^{T} E\left\{\rho(t) V(t)^{2}-\lambda(t) V(t)\right\} .
$$

Considerando o problema intermediário iniciando no instante $k$, a função valor para o problema auxiliar no tempo $k \in\{0, \ldots, T-1\}$ é definida por:

$$
J^{*}(V(k), \theta(k), k)=\min _{u(k) \in \mathcal{U}_{k}} \sum_{t=k}^{T} E\left\{\rho(t) V(t)^{2}-\lambda(t) V(t) \mid \mathcal{F}_{k}\right\},
$$

onde $\mathcal{U}_{k}=\left\{u_{k}=(u(k), \ldots, u(T-1))^{\prime} ; u(t)=\left(u_{1}(t), \ldots, u_{n}(t)\right)^{\prime}\right.$ é $\mathcal{F}_{t}$ mensurável para cada $t=k, \ldots, T-1\}$.

Com a definição da função custo do problema, passa-se à derivação da lei de controle ótima que é solução do problema auxiliar $A(\lambda, \rho)$ de média-variância a tempo discreto com saltos. Como nos problemas estocásticos lineares quadráticos clássicos, a lei de controle ótima depende da solução de um conjunto de equações recursivas a diferença de Riccati interconectadas.

Sejam os vetores $m$-dimensionais $K(t)=\left(K_{1}(t), \ldots, K_{m}(t)\right)^{\prime}, Z(t)=\left(Z_{1}(t)\right.$, $\left.\ldots, Z_{m}(t)\right)^{\prime}$ e $D(t)=\left(D_{1}(t), \ldots, D_{m}(t)\right)^{\prime}$, onde $K_{i}(t), Z_{i}(t)$ e $D_{i}(t)$ são calculados recursivamente de trás para frente através das equações a diferença interconectadas de Riccati:

$$
\left\{\begin{array}{l}
K_{i}(t)=\rho(t)+Q_{i}(t) \mathcal{E}_{i}[K(t+1)], \quad K_{i}(T)=\rho(T), \\
Z_{i}(t)=-\lambda(t)+R_{i}(t) \mathcal{E}_{i}[Z(t+1)], \quad Z_{i}(T)=-\lambda(T), \\
D_{i}(t)=-\frac{\mathcal{E}_{i}[Z(t+1)]^{2}}{4 \mathcal{E}_{i}[K(t+1)]} \beta_{i}(t)+\mathcal{E}_{i}[D(t+1)], \quad D_{i}(T)=0,
\end{array}\right.
$$

para $t=T-1, \ldots, 0$ e $i \in \mathcal{M}$.

Para $s, t=0, \ldots, T$, com $s \leq t$, convenientemente definem-se $\mathcal{Q}(t, s) \in \mathbb{R}^{m \times m}$, 
$\mathcal{R}(t, s) \in \mathbb{R}^{m \times m} \mathcal{K}(t, s) \in \mathbb{R}^{m}$ e $\mathcal{Z}(t, s) \in \mathbb{R}^{m}$ como:

$$
\begin{aligned}
\mathcal{Q}(t, s) & =\prod_{k=s}^{t-1} Q(k) P(k), \mathcal{K}(t, s)=\mathcal{Q}(t, s) e, \mathcal{K}(s)=\mathcal{K}(T, s), \\
\mathcal{R}(t, s) & =\prod_{k=s}^{t-1} R(k) P(k), \mathcal{Z}(t, s)=\mathcal{R}(t, s) e, \mathcal{Z}(s)=\mathcal{Z}(T, s),
\end{aligned}
$$

onde $\mathcal{Q}(s, s)=I$ e $\mathcal{R}(s, s)=I$. A partir de (4.2) deriva-se que

$$
K(t)=\sum_{s=t}^{T} \rho(s) \mathcal{K}(s, t)
$$

e

$$
Z(t)=-\sum_{s=t}^{T} \lambda(s) \mathcal{Z}(s, t)
$$

Apresenta-se então o seguinte teorema com a estratégia ótima de investimentos ou lei de controle ótima.

Teorema 4.1 A lei de controle ótima $u^{*}=(u(0), \ldots, u(T-1))$ para o problema (3.39), é dada como:

$$
u(t)=-\phi_{\theta(t)}(t)^{-1} \varphi_{\theta(t)}(t) V(t)-\frac{\mathcal{E}_{\theta(t)}[Z(t+1)]}{2 \mathcal{E}_{\theta(t)}[K(t+1)]} \phi_{\theta(t)}(t)^{-1} \chi_{\theta(t)}(t)
$$

Além disso, a função valor para o problema intermediário (4.1) é expressa como:

$$
J^{*}(V(t), \theta(t), t)=K_{\theta(t)}(t) V(t)^{2}+Z_{\theta(t)}(t) V(t)+D_{\theta(t)}(t) .
$$

Prova. Aplica-se indução em $t$. Para $t=T$, tem-se que:

$$
\begin{aligned}
J^{*}(V(T), \theta(T), T) & =\min _{u(T) \in \mathcal{U}_{T}} \sum_{t=T}^{T} E\left\{\rho(t) V(t)^{2}-\lambda(t) V(t) \mid \mathcal{F}_{T}\right\} \\
& =\rho(T) V(T)^{2}-\lambda(T) V(T) \\
& =K_{\theta(T)}(T) V(T)^{2}+Z_{\theta(T)}(T) V(T)+D_{\theta(T)}(T),
\end{aligned}
$$


satisfazendo o Teorema 4.1 .

Supondo que o resultado atenda ao Teorema $4.1 \mathrm{em} t=k+1$. Mostra-se que para $t=k$, (4.6) continua válida. Assim, para $\theta(k)=i \in \mathcal{M}$ e $E(V(k))=v$, segue, através do princípio de otimalidade de Bellman, que:

$$
\begin{aligned}
J^{*}(v, i, k)= & \min _{u(k) \in \mathcal{U}_{k}} \sum_{t=k}^{T} E\left\{\rho(t) V(t)^{2}-\lambda(t) V(t) \mid \mathcal{F}_{k}\right\} \\
= & \min _{u(k) \in \mathcal{U}_{k}} E\left\{J^{*}(V(k+1), \theta(k+1), k+1) \mid \mathcal{F}_{k}\right\} \\
& +\rho(k) v^{2}-\lambda(k) v .
\end{aligned}
$$

Substituindo (4.6) na equação acima, obtém-se:

$$
\begin{aligned}
J^{*}(v, i, k)= & \min _{u(k) \in \mathcal{U}_{k}} E\left\{K_{\theta(k+1)}(k+1) V(k+1)^{2}+Z_{\theta(k+1)}(k+1) V(k+1)\right. \\
& \left.+D_{\theta(k+1)}(k+1) \mid \mathcal{F}_{k}\right\}+\rho(k) v^{2}-\lambda(k) v .
\end{aligned}
$$

Utilizando (3.14), encontra-se que:

$$
\begin{aligned}
J^{*}(v, i, k)= & \min _{u(k) \in \mathcal{U}_{k}} E\left\{\left[K_{\theta(k+1)}(k+1)\right] \cdot\left[A_{\theta(k)}(k) V(k)+B_{\theta(k)}(k)^{\prime} u(k)\right]^{2}\right. \\
& \left.+Z_{\theta(k+1)}(k+1)\left[A_{\theta(k)}(k) V(k)+B_{\theta(k)}(k)^{\prime} u\right]+D_{\theta(k+1)}(k+1) \mid \mathcal{F}_{k}\right\} \\
& +\rho(k) v^{2}-\lambda(k) v \\
= & \min _{u(k) \in \mathcal{U}_{k}} \mathcal{E}_{i}(K(k+1))\left[E\left[A_{i}^{2}(k)\right] v^{2}+2 E\left[A_{i}(k) B_{i}(k)^{\prime}\right]\right. \\
& \left.\cdot u(k) v+u(k)^{\prime} E\left[B_{i}(k) B_{i}(k)^{\prime}\right] u(k)\right]+\mathcal{E}_{i}(Z(k+1)) \\
& \cdot\left(E\left[A_{i}(k)\right] v+E\left[B_{i}(k)^{\prime}\right] u(k)\right)+\mathcal{E}_{i}(D(k+1)) \\
& +\rho(k) v^{2}-\lambda(k) v,
\end{aligned}
$$


$\mathrm{Ou}$

$$
\begin{aligned}
J^{*}(v, i, k)= & \min _{u(k) \in \mathcal{U}_{k}} \mathcal{E}_{i}[K(k+1)]\left[\delta_{i}(k) v^{2}+2 \varphi_{i}(k)^{\prime} u(k) v+u(k)^{\prime} \phi_{i}(k) u(k)\right] \\
& +\mathcal{E}_{i}(Z(k+1))\left(\hat{A}_{i}(k) v+\chi_{i}(k)^{\prime} u(k)\right)+\mathcal{E}_{i}(D(k+1)) \\
& +\rho(k) v^{2}-\lambda(k) v .
\end{aligned}
$$

Tomando-se a derivada de (4.7) em relação a $u(k)$ e igualando o resultado a zero, encontra-se:

$$
2 \mathcal{E}_{i}[K(k+1)]\left(\varphi_{i}(k) v+\phi_{i}(k) u(k)\right)+\mathcal{E}_{i}[Z(k+1)] \chi_{i}(k)=0 .
$$

Portanto:

$$
u(k)=-\phi_{i}(k)^{-1} \varphi_{i}(k) v-\frac{\mathcal{E}_{i}[Z(k+1)]}{2 \mathcal{E}_{i}[K(k+1)]} \phi_{i}(k)^{-1} \chi_{i}(k),
$$

como em (4.5).

Substituindo (4.8) em (4.7), chega-se a:

$$
\begin{aligned}
J^{*}(v, i, k)= & \left\{\mathcal { E } _ { i } [ K ( k + 1 ) ] \left[\delta_{i}(k) v^{2}+2 \varphi_{i}(k)^{\prime}\right.\right. \\
& \cdot\left(-\phi_{i}(k)^{-1} \varphi_{i}(k) v-\frac{\mathcal{E}_{i}[Z(k+1)]}{2 \mathcal{E}_{i}[K(k+1)]} \phi_{i}(k)^{-1} \chi_{i}(k)\right) v \\
& +\left(-\phi_{i}(k)^{-1} \varphi_{i}(k) v-\frac{\mathcal{E}_{i}[Z(k+1)]}{2 \mathcal{E}_{i}[K(k+1)]} \phi_{i}(k)^{-1} \chi_{i}(k)\right)^{\prime} \phi_{i}(k) \\
& \left.\cdot\left(-\phi_{i}(k)^{-1} \varphi_{i}(k) v-\frac{\mathcal{E}_{i}[Z(k+1)]}{2 \mathcal{E}_{i}[K(k+1)]} \phi_{i}(k)^{-1} \chi_{i}(k)\right)\right] \\
& +\mathcal{E}_{i}[Z(k+1)] \hat{A}_{i}(k) v+\mathcal{E}_{i}[Z(k+1)] \chi_{i}(k)^{\prime} \\
& \cdot\left(-\phi_{i}(k)^{-1} \varphi_{i}(k) v-\frac{\mathcal{E}_{i}[Z(k+1)]}{2 \mathcal{E}_{i}[K(k+1)]} \phi_{i}(k)^{-1} \chi_{i}(k)\right) \\
& \left.+\mathcal{E}_{i}[D(k+1)]+\rho(k) v^{2}-\lambda(k) v\right\},
\end{aligned}
$$




$$
\begin{aligned}
J^{*}(v, i, k)= & \left\{\mathcal { E } _ { i } [ K ( k + 1 ) ] \left[\delta_{i}(k) v^{2}-2 \varphi_{i}(k)^{\prime} \phi_{i}(k)^{-1} \varphi_{i}(k) v^{2}-2 \varphi_{i}(k)^{\prime}\right.\right. \\
& \cdot \frac{\mathcal{E}_{i}[Z(k+1)]}{2 \mathcal{E}_{i}[K(k+1)]} \phi_{i}(k)^{-1} \chi_{i}(k) v+\varphi_{i}(k)^{\prime} \phi_{i}(k)^{-1} v \phi_{i}(k) \phi_{i}(k)^{-1} \\
& \cdot \varphi_{i}(k) v+\varphi_{i}(k)^{\prime} \phi_{i}(k)^{-1} v \phi_{i}(k) \frac{\mathcal{E}_{i}[Z(k+1)]}{2 \mathcal{E}_{i}[K(k+1)]} \phi_{i}(k)^{-1} \chi_{i}(k) \\
& +\frac{\mathcal{E}_{i}[Z(k+1)]}{2 \mathcal{E}_{i}[K(k+1)]} \chi_{i}(k)^{\prime} \phi_{i}(k)^{-1} \phi_{i}(k) \phi_{i}(k)^{-1} \varphi_{i}(k) v \\
& +\frac{\mathcal{E}_{i}[Z(k+1)]}{2 \mathcal{E}_{i}[K(k+1)]} \chi_{i}(k)^{\prime} \phi_{i}(k)^{-1} \phi_{i}(k) \frac{\mathcal{E}_{i}[Z(k+1)]}{2 \mathcal{E}_{i}[K(k+1)]} \phi_{i}(k)^{-1} \\
& \left.\cdot \chi_{i}(k)\right]+\mathcal{E}_{i}[Z(k+1)] \hat{A}_{i}(k) v-\mathcal{E}_{i}[Z(k+1)] \chi_{i}(k)^{\prime} \phi_{i}(k)^{-1} \\
& \cdot \varphi_{i}(k) v-\mathcal{E}_{i}[Z(k+1)] \chi_{i}(k)^{\prime} \frac{\mathcal{E}_{i}[Z(k+1)]}{2 \mathcal{E}_{i}[K(k+1)]} \phi_{i}(k)^{-1} \chi_{i}(k) \\
& \left.+\mathcal{E}_{i}[D(k+1)]+\rho(k) v^{2}-\lambda(k) v\right\} .
\end{aligned}
$$

Logo,

$$
\begin{aligned}
J^{*}(v, i, k)= & \left\{\mathcal{E}_{i}[K(k+1)] \delta_{i}(k) v^{2}-2 \mathcal{E}_{i}[K(k+1)] \varphi_{i}(k)^{\prime} \phi_{i}(k)^{-1} \varphi_{i}(k) v^{2}\right. \\
& -\mathcal{E}_{i}[Z(k+1)] \varphi_{i}(k)^{\prime} \phi_{i}(k)^{-1} \chi_{i}(k) v+\mathcal{E}_{i}[K(k+1)] \varphi_{i}(k)^{\prime} \\
& \cdot \phi_{i}(k)^{-1} \varphi_{i}(k) v^{2}+\frac{\mathcal{E}_{i}[Z(k+1)]}{2} \varphi_{i}(k)^{\prime} \phi_{i}(k)^{-1} \chi_{i}(k) v \\
& +\frac{\mathcal{E}_{i}[Z(k+1)]}{2} \chi_{i}(k)^{\prime} \phi_{i}(k)^{-1} \varphi_{i}(k) v+\frac{\mathcal{E}_{i}[Z(k+1)]^{2}}{4 \mathcal{E}_{i}[K(k+1)]} \\
& \cdot \chi_{i}(k)^{\prime} \phi_{i}(k)^{-1} \chi_{i}(k)+\mathcal{E}_{i}[Z(k+1)] \hat{A}_{i}(k) v-\mathcal{E}_{i}[Z(k+1)] \\
& \cdot \chi_{i}(k)^{\prime} \phi_{i}(k)^{-1} \varphi_{i}(k) v-\frac{\mathcal{E}_{i}[Z(k+1)]^{2}}{2 \mathcal{E}_{i}[K(k+1)]} \chi_{i}(k)^{\prime} \phi_{i}(k)^{-1} \chi_{i}(k) \\
& \left.+\mathcal{E}_{i}[D(k+1)]+\rho(k) v^{2}-\lambda(k) v\right\} \\
= & \left\{\mathcal{E}_{i}[K(k+1)] \delta_{i}(k) v^{2}-\mathcal{E}_{i}[K(k+1)] \varphi_{i}(k)^{\prime} \phi_{i}(k)^{-1} \varphi_{i}(k) v^{2}\right. \\
& -\mathcal{E}_{i}[Z(k+1)] \chi_{i}(k)^{\prime} \phi_{i}(k)^{-1} \varphi_{i}(k) v+\mathcal{E}_{i}[Z(k+1)] \hat{A}_{i}(k) v \\
& -\frac{\mathcal{E}_{i}[Z(k+1)]^{2}}{4 \mathcal{E}_{i}[K(k+1)]} \chi_{i}(k)^{\prime} \phi_{i}(k)^{-1} \chi_{i}(k) \\
& \left.+\mathcal{E}_{i}[D(k+1)]+\rho(k) v^{2}-\lambda(k) v\right\}
\end{aligned}
$$


ou seja,

$$
\begin{aligned}
J^{*}(v, i, k)= & \left(\mathcal{E}_{i}[K(k+1)] Q_{i}(t)+\rho(k)\right) v^{2}+\left(\mathcal{E}_{i}[Z(k+1)] R_{i}(t)-\lambda(k)\right) v \\
& -\frac{\mathcal{E}_{i}[Z(k+1)]^{2}}{4 \mathcal{E}_{i}[K(k+1)]} \beta_{i}(t)+\mathcal{E}_{i}[D(k+1)],
\end{aligned}
$$

produzindo o resultado apresentado em (4.6) para a função valor.

\subsection{Resultados da Formulação Auxiliar}

A partir da lei de controle ótimo encontrada na seção anterior é possível derivar importantes resultados para o problema auxiliar $A(\lambda, \rho)$. Nesta seção, deriva-se analiticamente as equações para o valor esperado e para a variância do valor terminal da riqueza sob a lei de controle ótima (4.5).

Antes de apresentar os resultados, define-se para $k=0, \ldots, T-1$ e $\kappa=0, \ldots, \iota_{f}-1$ :

$$
\begin{aligned}
\mathrm{B}(k)= & P(k)^{\prime} \operatorname{diag}\left(\frac{\pi_{i}(k) \beta_{i}(k)}{\mathcal{E}_{i}(K(k+1))}\right) P(k), \\
\mathcal{B}\left(\tau_{\kappa}\right)= & \sum_{k=\tau_{\kappa}}^{\tau_{\kappa+1}-1} \mathcal{R}\left(\tau_{\kappa+1}, k+1\right)^{\prime} \mathrm{B}(k) \mathcal{R}\left(\tau_{\kappa+1}, k+1\right), \\
\mathcal{C}\left(\tau_{\kappa}\right)= & \sum_{k=\tau_{\kappa}}^{\tau_{\kappa+1}-1} \mathcal{Q}\left(\tau_{\kappa+1}, k+1\right)^{\prime} P(k)^{\prime} \\
& \cdot \operatorname{diag}\left(\pi_{i}(k) \beta_{i}(k)\left(\frac{\mathcal{E}_{i}\left(\mathcal{R}\left(\tau_{\kappa+1}, k+1\right)\right)}{\mathcal{E}_{i}(K(k+1))}\right)^{2}\right) .
\end{aligned}
$$

Seja, para $j \in \mathcal{M}$,

$$
q(t)=\left(q_{1}(t), \ldots, q_{m}(t)\right)^{\prime}, \operatorname{com} q_{j}(t)=E\left(V(t) 1_{\{\theta(t)=j\}}\right)
$$

e

$$
g(t)=\left(g_{1}(t), \ldots, g_{m}(t)\right)^{\prime}, \operatorname{com} g_{j}(t)=E\left(V(t)^{2} 1_{\{\theta(t)=j\}}\right)
$$


O seguinte teorema apresenta as formulações analíticas para a esperança e para a variância da riqueza do investidor em cada momento $\tau_{\kappa} \in \mathcal{I}$.

Teorema 4.2 Sob a lei de controle ótima (4.5), o valor esperado e a variância do patrimônio do investidor em cada instante de tempo $\tau_{\kappa} \in \mathcal{I}$ são expressos, respectivamente, como:

$$
E\left(V\left(\tau_{\kappa}\right)\right)=q(0)^{\prime} \mathcal{Z}\left(\tau_{\kappa}, 0\right)-\frac{1}{2} \sum_{k=0}^{\kappa-1} Z\left(\tau_{k+1}\right)^{\prime} \mathcal{B}\left(\tau_{k}\right)^{\prime} e
$$

$e$

$$
\begin{aligned}
\operatorname{Var}\left(V\left(\tau_{\kappa}\right)\right)= & g(0)^{\prime} \mathcal{K}\left(\tau_{\kappa}, 0\right)+\frac{1}{4} \sum_{k=0}^{\kappa-1} \operatorname{diag}\left(Z_{i}\left(\tau_{k+1}\right)^{2}\right)^{\prime} \mathcal{C}(k)^{\prime} e \\
& -\left(q(0)^{\prime} \mathcal{Z}\left(\tau_{\kappa}, 0\right)-\frac{1}{2} \sum_{k=0}^{\kappa-1} Z\left(\tau_{k+1}\right)^{\prime} \mathcal{B}\left(\tau_{k}\right)^{\prime} e\right)^{2}
\end{aligned}
$$

Prova. Inicialmente, encontram-se as expressões para as esperanças do valor da riqueza e do quadrado deste sob a lei de controle ótimo (4.5), a qual se repete abaixo:

$$
u^{*}(t)=-\phi_{\theta(t)}(t)^{-1} \varphi_{\theta(t)}(t) V(t)-\frac{\mathcal{E}_{\theta(t)}[Z(t+1)]}{2 \mathcal{E}_{\theta(t)}[K(t+1)]} \phi_{\theta(t)}(t)^{-1} \chi_{\theta(t)}(t) .
$$

Utilizando esta política ótima de investimento em (3.14), obtém-se:

$$
\begin{aligned}
V(t+1)= & A_{\theta(t)}(t) V(t)+B_{\theta(t)}(t)^{\prime} \\
& \cdot\left(-\phi_{\theta(t)}(t)^{-1} \varphi_{\theta(t)}(t) V(t)-\frac{\mathcal{E}_{\theta(t)}[Z(t+1)]}{2 \mathcal{E}_{\theta(t)}[K(t+1)]} \phi_{\theta(t)}(t)^{-1} \chi_{\theta(t)}(t)\right)
\end{aligned}
$$

$\mathrm{Ou}$

$$
\begin{aligned}
V(t+1)= & \left(A_{\theta(t)}(t)-B_{\theta(t)}(t)^{\prime} \phi_{\theta(t)}(t)^{-1} \varphi_{\theta(t)}(t)\right) V(t) \\
& -\frac{\mathcal{E}_{\theta(t)}[Z(t+1)]}{2 \mathcal{E}_{\theta(t)}[K(t+1)]} B_{\theta(t)}(t)^{\prime} \phi_{\theta(t)}(t)^{-1} \chi_{\theta(t)}(t),
\end{aligned}
$$

que representa o processo da riqueza quando adotada a estratégia $u^{*}(t)$. 
Conforme em Costa et al. (2005), (4.12) e (4.16), segue que:

$$
\begin{aligned}
q_{j}(t+1)= & \sum_{i=1}^{m} E\left[V(t+1) 1_{\{\theta(t+1)=j\}} 1_{\{\theta(t)=i\}}\right] \\
= & \sum_{i=1}^{m} E\left[\left(A_{\theta(t)}(t)-B_{\theta(t)}(t)^{\prime} \phi_{\theta(t)}(t)^{-1} \varphi_{\theta(t)}(t)\right) V(t) 1_{\{\theta(t+1)=j\}} 1_{\{\theta(t)=i\}}\right] \\
& -\sum_{i=1}^{m} E\left[\frac{\mathcal{E}_{\theta(t)}[Z(t+1)]}{2 \mathcal{E}_{\theta(t)}[K(t+1)]} B_{\theta(t)}(t)^{\prime} \phi_{\theta(t)}(t)^{-1} \chi_{\theta(t)}(t) 1_{\{\theta(t+1)=j\}} 1_{\{\theta(t)=i\}}\right] \\
= & \sum_{i=1}^{m}\left(A_{i}(t)-\chi_{i}(t)^{\prime} \phi_{i}(t)^{-1} \varphi_{i}(t)\right) \cdot E\left[V(t) 1_{\{\theta(t)=i\}} P\left(\theta(t+1)=j \mid \mathcal{F}_{t}\right)\right] \\
& -\sum_{i=1}^{m} \frac{\mathcal{E}_{i}[Z(t+1)]}{2 \mathcal{E}_{i}[K(t+1)]} \chi_{i}(t)^{\prime} \phi_{i}(t)^{-1} \chi_{i}(t) \cdot E\left[1_{\{\theta(t)=i\}} P\left(\theta(t+1)=j \mid \mathcal{F}_{t}\right)\right]
\end{aligned}
$$

$\mathrm{Ou}$

$$
q_{j}(t+1)=\sum_{i=1}^{m} p_{i j}(t) R_{i}(t) q_{i}(t)-\frac{1}{2} \sum_{i=1}^{m} p_{i j}(t) \pi_{i}(t) \frac{\mathcal{E}_{i}[Z(t+1)]}{\mathcal{E}_{i}[K(t+1)]} \beta_{i}(t) .
$$

Elevando-se ao quadrado os dois lados de (4.16) obtém-se:

$$
\begin{aligned}
V(t+1)^{2}= & {\left[\left(A_{\theta(t)}(t)-B_{\theta(t)}(t)^{\prime} \phi_{\theta(t)}(t)^{-1} \varphi_{\theta(t)}(t)\right) V(t)\right.} \\
& \left.-\frac{\mathcal{E}_{\theta(t)}(Z(t+1))}{2 \mathcal{E}_{\theta(t)}(K(t+1))} B_{\theta(t)}(t)^{\prime} \phi_{\theta(t)}(t)^{-1} \chi_{\theta(t)}(t)\right]^{2} \\
= & \left(A_{\theta(t)}(t)^{2}-2 A_{\theta(t)}(t) B_{\theta(t)}(t)^{\prime} \phi_{\theta(t)}(t)^{-1} \varphi_{\theta(t)}(t)\right. \\
& \left.+\varphi_{\theta(t)}(t)^{\prime} \phi_{\theta(t)}(t)^{-1} B_{\theta(t)}(t) B_{\theta(t)}(t)^{\prime} \phi_{\theta(t)}(t)^{-1} \varphi_{\theta(t)}(t)\right) V(t)^{2} \\
& -\left[\left(A_{\theta(t)}(t)-B_{\theta(t)}(t)^{\prime} \phi_{\theta(t)}(t)^{-1} \varphi_{\theta(t)}(t)\right)\right. \\
& \left.\cdot \frac{\mathcal{E}_{\theta(t)}[Z(t+1)]}{\mathcal{E}_{\theta(t)}[K(t+1)]} B_{\theta(t)}(t)^{\prime} \phi_{\theta(t)}(t)^{-1} \chi_{\theta(t)}(t)\right] V(t) \\
& +\frac{1}{4}\left(\frac{\mathcal{E}_{\theta(t)}[Z(t+1)]}{\mathcal{E}_{\theta(t)}[K(t+1)]}\right)^{2} \chi_{\theta(t)}(t)^{\prime} \phi_{\theta(t)}(t)^{-1} B_{\theta(t)}(t) B_{\theta(t)}(t)^{\prime} \\
& \cdot \phi_{\theta(t)}(t)^{-1} \chi_{\theta(t)}(t) .
\end{aligned}
$$


A partir de Costa et al. (2005), (4.13) e (4.18), tem-se que:

$$
\begin{aligned}
g_{j}(t+1)= & \sum_{i=1}^{m} E\left[V(t+1)^{2} 1_{\{\theta(t+1)=j\}} 1_{\{\theta(t)=i\}}\right] \\
= & \sum_{i=1}^{m} E\left[\left(A_{\theta(t)}(t)^{2}-2 A_{\theta(t)}(t) B_{\theta(t)}(t)^{\prime} \phi_{\theta(t)}(t)^{-1} \varphi_{\theta(t)}(t)+\varphi_{\theta(t)}(t)^{\prime}\right.\right. \\
& \left.\left.\cdot \phi_{\theta(t)}(t)^{-1} B_{\theta(t)}(t) B_{\theta(t)}(t)^{\prime} \phi_{\theta(t)}(t)^{-1} \varphi_{\theta(t)}(t)\right) V(t)^{2} 1_{\{\theta(t+1)=j\}} 1_{\{\theta(t)=i\}}\right] \\
& -\sum_{i=1}^{m} E\left[\frac{\mathcal{E}_{\theta(t)}[Z(t+1)]}{\mathcal{E}_{\theta(t)}[K(t+1)]}\left(A_{\theta(t)}(t)-B_{\theta(t)}(t)^{\prime} \phi_{\theta(t)}(t)^{-1} \varphi_{V i}(t)\right)\right. \\
& \left.\cdot B_{\theta(t)}(t)^{\prime} \phi_{\theta(t)}(t)^{-1} \chi_{\theta(t)}(t) V(t) 1_{\{\theta(t+1)=j\}} 1_{\{\theta(t)=i\}}\right] \\
& +\sum_{i=1}^{m} E\left[\frac{1}{4}\left(\frac{\mathcal{E}_{\theta(t)}[Z(t+1)]}{\mathcal{E}_{\theta(t)}[K(t+1)]}\right)^{2} \chi_{\theta(t)}(t)^{\prime}\right. \\
& \left.\cdot \phi_{\theta(t)}(t)^{-1} B_{\theta(t)}(t) B_{\theta(t)}(t)^{\prime} \phi_{\theta(t)}(t)^{-1} \chi_{\theta(t)}(t) 1_{\{\theta(t+1)=j\}} 1_{\{\theta(t)=i\}}\right] \\
= & \sum_{i=1}^{m}\left(A_{i}(t)^{2}-2 \varphi_{i}(t)^{\prime} \phi_{i}(t)^{-1} \varphi_{i}(t)+\varphi_{i}(t)^{\prime} \phi_{i}(t)^{-1} \varphi_{i}(t)\right) \\
& \cdot E\left[V(t)^{2} 1_{\{\theta(t)=i\}} P\left(\theta(t+1)=j \mid \mathcal{F}_{t}\right)\right]-\sum_{i=1}^{m} \frac{\mathcal{E}_{i}[Z(t+1)]}{\mathcal{E}_{i}[K(t+1)]} \\
& \cdot\left(\varphi_{i}(t)^{\prime} \phi_{i}(t)^{-1} \chi_{i}(t)-\varphi_{i}(t)^{\prime} \phi_{i}(t)^{-1} \phi_{i}(t) \phi_{i}(t)^{-1} \chi_{i}(t)\right) \\
& \cdot E\left[V(t) 1_{\{\theta(t)=i\}} P\left(\theta(t+1)=j \mid \mathcal{F}_{t}\right)\right]+\frac{1}{4} \sum_{i=1}^{m}\left(\frac{\mathcal{E}_{i}[Z(t+1)]}{\mathcal{E}_{i}[K(t+1)]}\right)^{2} \\
& \cdot \chi_{i}(t)^{\prime} \phi_{i}(t)^{-1} \phi_{i}(t) \phi_{i}(t)^{-1} \chi_{i}(t) E\left[1_{\{\theta(t)=i\}} P\left(\theta(t+1)=j \mid \mathcal{F}_{t}\right)\right] \\
& \\
& \\
&
\end{aligned}
$$

$\mathrm{Ou}$

$$
g_{j}(t+1)=\sum_{i=1}^{m} p_{i j}(t) Q_{i}(t) g_{i}(t)+\frac{1}{4} \sum_{i=1}^{m} p_{i j}(t) \pi_{i}(t)\left(\frac{\mathcal{E}_{i}[Z(t+1)]}{\mathcal{E}_{i}[K(t+1)]}\right)^{2} \beta_{i}(t)
$$

Para duas sequências de vetores em $\mathbb{R}^{m}, v(t)=\left(v_{1}(t), \ldots, v_{m}(t)\right)^{\prime}$ e $\vartheta(t)=\left(\vartheta_{1}(t)\right.$, $\left.\ldots, \vartheta_{m}(t)\right)^{\prime}$, para $t=1, \ldots, T$, definem-se para $t=0, \ldots, T-1$, os vetores $h(v, \vartheta, t)=$ $\left(h_{1}(v, \vartheta, t), \ldots, h_{m}(v, \vartheta, t)\right)^{\prime}$ e $r(v, \vartheta, t)=\left(r_{1}(v, \vartheta, t), \ldots, r_{m}(v, \vartheta, t)\right)^{\prime}$ em $\mathbb{R}^{m}$ como:

$$
\begin{aligned}
& h_{j}(v, \vartheta, t)=\frac{1}{2} \sum_{i=1}^{m} p_{i j} \pi_{i}(t) \frac{\mathcal{E}_{i}[v(t+1)]}{\mathcal{E}_{i}[\vartheta(t+1)]} \beta_{i}(t), \\
& r_{j}(v, \vartheta, t)=\frac{1}{4} \sum_{i=1}^{m} p_{i j} \pi_{i}(t)\left(\frac{\mathcal{E}_{i}[v(t+1)]}{\mathcal{E}_{i}[\vartheta(t+1)]}\right)^{2} \beta_{i}(t) .
\end{aligned}
$$


Substituindo os vetores (4.20) e (4.21) em (4.17) e (4.19), respectivamente, encontramse as seguintes equações recursivas para $s, t=0, \ldots, T$, com $s<t$ :

$$
q(t)^{\prime}=q(s)^{\prime} \mathcal{R}(t, s)-\sum_{k=s}^{t-1} h(Z, K, k)^{\prime} \mathcal{R}(t, k+1)
$$

e

$$
g(t)^{\prime}=g(s)^{\prime} \mathcal{Q}(t, s)+\sum_{k=s}^{t-1} r(Z, K, k)^{\prime} \mathcal{Q}(t, k+1) .
$$

De (4.9), (4.22) e (4.23) tem-se, respectivamente, que para $\kappa=0, \ldots, \iota_{f}-1$ e para $t=0, \ldots, T-1$

$$
q\left(\tau_{\kappa+1}\right)=\mathcal{R}\left(\tau_{\kappa+1}, \tau_{\kappa}\right)^{\prime} q\left(\tau_{\kappa}\right)-\frac{1}{2} \sum_{k=\tau_{\kappa}}^{\tau_{\kappa+1}-1} \mathcal{R}\left(\tau_{\kappa+1}, k+1\right)^{\prime} \mathrm{B}(k) Z(k+1)
$$

$\mathrm{e}$

$$
\begin{aligned}
g\left(\tau_{\kappa+1}\right)= & \mathcal{Q}\left(\tau_{\kappa+1}, \tau_{\kappa}\right)^{\prime} g\left(\tau_{\kappa}\right)+\frac{1}{4} \sum_{k=\tau_{\kappa}}^{\tau_{\kappa+1}-1} \mathcal{Q}\left(\tau_{\kappa+1}, k+1\right)^{\prime} P(k)^{\prime} \\
& \cdot \operatorname{diag}\left(\pi_{i}(k) \beta_{i}(k)\left(\frac{\mathcal{E}_{i}(Z(k+1))}{\mathcal{E}_{i}(K(k+1))}\right)^{2}\right) .
\end{aligned}
$$

Lembrando que $\lambda(t)=0$ para $t \notin \mathcal{I}$, segue de (4.2) que para $\tau_{\kappa} \leq k \leq \tau_{\kappa+1}-1$

$$
Z(k+1)=\mathcal{R}\left(\tau_{\kappa+1}, k+1\right) Z\left(\tau_{\kappa+1}\right)
$$

$\mathrm{e}$

$$
Z\left(\tau_{\kappa}\right)=-\lambda\left(\tau_{\kappa}\right) e+\mathcal{R}\left(\tau_{\kappa+1}, \tau_{\kappa}\right) Z\left(\tau_{\kappa+1}\right)
$$

Substituindo (4.10) e (4.26) em (4.24), obtém-se

$$
q\left(\tau_{\kappa+1}\right)=\mathcal{R}\left(\tau_{\kappa+1}, \tau_{\kappa}\right)^{\prime} q\left(\tau_{\kappa}\right)-\frac{1}{2} \mathcal{B}\left(\tau_{\kappa}\right) Z\left(\tau_{\kappa+1}\right) .
$$


Da mesma forma, substituindo (4.11) e (4.26) em (4.25), resulta que

$$
\begin{aligned}
g\left(\tau_{\kappa+1}\right)= & \mathcal{Q}\left(\tau_{\kappa+1}, \tau_{\kappa}\right)^{\prime} g\left(\tau_{\kappa}\right)+\frac{1}{4} \sum_{k=\tau_{\kappa}}^{\tau_{\kappa+1}-1} \mathcal{Q}\left(\tau_{\kappa+1}, k+1\right)^{\prime} P(k)^{\prime} \\
& \cdot \operatorname{diag}\left(\pi_{i}(k) \beta_{i}(k)\left(\frac{\mathcal{E}_{i}\left(\mathcal{R}\left(\tau_{\kappa+1}, k+1\right)\right)}{\mathcal{E}_{i}(K(k+1))}\right)^{2}\right) \operatorname{diag}\left(Z_{i}\left(\tau_{\kappa+1}\right)^{2}\right) \\
= & \mathcal{Q}\left(\tau_{\kappa+1}, \tau_{\kappa}\right)^{\prime} g\left(\tau_{\kappa}\right)+\frac{1}{4} \mathcal{C}\left(\tau_{\kappa}\right) \operatorname{diag}\left(Z_{i}\left(\tau_{\kappa+1}\right)^{2}\right) .
\end{aligned}
$$

Como $E(V(k))=q(k)^{\prime}$ e e $E\left(V(k)^{2}\right)=g(k)^{\prime} e$, de (4.28) e (4.29) são obtidas as expressões desejadas para a esperança do valor e do quadrado do valor da riqueza no instante $\tau_{\kappa} \in \mathcal{I}$, respectivamente como:

$$
E\left(V\left(\tau_{\kappa}\right)\right)=q(0)^{\prime} \mathcal{Z}\left(\tau_{\kappa}, 0\right)-\frac{1}{2} \sum_{k=0}^{\kappa-1} Z\left(\tau_{k+1}\right)^{\prime} \mathcal{B}\left(\tau_{k}\right)^{\prime} e
$$

$\mathrm{e}$

$$
E\left(V\left(\tau_{\kappa}\right)^{2}\right)=g(0)^{\prime} \mathcal{K}\left(\tau_{\kappa}, 0\right)+\frac{1}{4} \sum_{k=0}^{\kappa-1} \operatorname{diag}\left(Z_{i}\left(\tau_{k+1}\right)^{2}\right)^{\prime} \mathcal{C}(k)^{\prime} e .
$$

A formulação da variância da riqueza em cada instante de tempo $\left(\tau_{\kappa} \in \mathcal{I}\right)$ em (4.15) é resultante de (4.14) e (4.30), completando a prova.

\subsection{Solução para o Problema Generalizado de Média-Variância}

Nesta seção, resolve-se o problema geral de média-variância $\operatorname{PGMV}(\rho, \ell, \nu)$. Mostra-se que a solução deste problema segue a partir da solução do problema auxiliar $A(\lambda, \rho)$ encontrada nas seções anteriores, utilizando a lei de controle (4.5). Na Subseção 4.3.1 é apresentada uma condição necessária quando $u \in P G M V(\rho, \ell, \nu)$, enquanto na Subseção 4.3.2, deriva-se uma condição suficiente para $u \in P G M V(\rho, \ell, \nu)$.

Denota-se por $\Pi(A(\lambda, \rho))$ e $\Pi(P G M V(\rho, \ell, \nu))$, respectivamente, o conjunto de soluções ótimas para os problemas $A(\lambda, \rho)$ e $\operatorname{PGMV}(\rho, \ell, \nu)$. 


\subsubsection{Condição Necessária}

Inicialmente, apresenta-se uma condição necessária quando $u \in \Pi(P G M V(\rho, \ell, \nu))$. A seguinte proposição é similar ao Teorema 1 em Li e Ng (2000) e em Zhu et al. (2004), e sua prova também é semelhante à encontrada nestes trabalhos.

Proposição 4.1 Se $u \in \Pi(P G M V(\rho, \ell, \nu))$ então $u \in \Pi(A(\lambda, \rho))$ com

$$
\lambda(t)=\ell(t)+2 \nu(t) E\left(V^{u}(t)\right),
$$

para $t=0, \ldots, T$.

Prova. Seja $u \in \Pi(P G M V(\rho, \ell, \nu))$ e, por contradição, assume-se que $u \notin \Pi(A(\lambda, \rho))$. Então existe $u^{*}$ tal que

$$
[\rho(1), \cdots, \rho(T),-\lambda(1), \cdots,-\lambda(T)]\left[\begin{array}{c}
E\left(V^{u^{*}}(1)^{2}\right) \\
\vdots \\
E\left(V^{u^{*}}(T)^{2}\right) \\
E\left(V^{u^{*}}(1)\right) \\
\vdots \\
E\left(V^{u^{*}}(T)\right)
\end{array}\right]
$$


Verifica-se de (3.32) que

$$
\begin{aligned}
& \frac{\partial P G M V(\rho, \ell, \nu)}{\partial E(V(t))}=\ell(t)+2 \nu(t) E\left(V^{u}(t)\right)=\lambda(t), \\
& \frac{\partial P G M V(\rho, \ell, \nu)}{\partial E\left(V(t)^{2}\right)}=-\rho(t) .
\end{aligned}
$$

Como PGMV $(\rho, \ell, \nu)$ é uma função convexa de $E(V(t))$ e $E\left(V(t)^{2}\right)$, é possível afirmar que:

$$
\begin{aligned}
\left.P G M V(\rho, \ell, \nu)\right|_{u^{*}} \geq & \left.P G M V(\rho, \ell, \nu)\right|_{u}+[\rho(1), \cdots, \rho(T),-\lambda(1), \cdots,-\lambda(T)] \\
& \cdot\left\{\left[\begin{array}{c}
E\left(V^{u^{*}}(1)^{2}\right) \\
\vdots \\
E\left(V^{u^{*}}(T)^{2}\right) \\
E\left(V^{u^{*}}(1)\right) \\
\vdots \\
E\left(V^{u^{*}}(T)\right)
\end{array}\right]-\left[\begin{array}{c}
E\left(V^{u}(1)^{2}\right) \\
\vdots \\
E\left(V^{u}(T)^{2}\right) \\
E\left(V^{u}(1)\right) \\
\vdots \\
E\left(V^{u}(T)\right)
\end{array}\right]\right\}
\end{aligned}
$$

Combinando (4.32) e (4.33), determina-se que:

$$
\left.P G M V(\rho, \ell, \nu)\right|_{u^{*}}>\left.P G M V(\rho, \ell, \nu)\right|_{u} .
$$

Esta conclusão contradiz a hipótese de que $u \in \Pi(P G M V(\rho, \ell, \nu))$, concluindo a prova.

Segue da Proposição 4.1 que para obter $u \in \Pi(P G M V(\rho, \ell, \nu))$, deve-se ter $u \in$ $\Pi(A(\lambda, \rho))$ dado por (4.5), com $\lambda(t)$ tal que (4.31) seja respeitada. Portanto, para encontrar $u \in \Pi(P G M V(\rho, \ell, \nu))$, dado pela expressão (4.5), depende-se da solução das equações de Riccati (4.2), que, por sua vez, dependem que o parâmetro auxiliar $\lambda(t)$ atenda a (4.31).

O procedimento aqui adotado para se chegar a $u$, com $\lambda(t)$ tal que (4.31) seja respeitada, é mais direto que o utilizado por Zhu et al. (2004). Em Zhu et al. (2004), 
os autores apresentam um conjunto de equações lineares que devem ser resolvidas para encontrar o parâmetro $\lambda(t)$ que atende a (4.31), e em seguida o substituem para a solução de equações recursivas a serem utilizadas na equação de controle. A proposição a seguir apresenta explicitamente a expressão de $Z(t)$, definido por (4.2), tal que (4.31) seja satisfeita, e a ser utilizada diretamente na equação de controle (4.5). Antes de apresentá-la, calcula-se recursivamente, para $\kappa=\iota_{f}-1, \ldots, 0$ as seguintes matrizes $\mathcal{G}\left(\tau_{\kappa}\right) \in \mathbb{R}^{m \times m}$ e vetores $\mathcal{S}\left(\tau_{\kappa}\right) \in \mathbb{R}^{m}$,

$$
\left\{\begin{array}{l}
\mathcal{G}\left(\tau_{\kappa}\right)=-2 \nu\left(\tau_{\kappa}\right) e e^{\prime}+\mathcal{R}\left(\tau_{\kappa+1}, \tau_{\kappa}\right)\left(I+\frac{1}{2} \mathcal{G}\left(\tau_{\kappa+1}\right) \mathcal{B}\left(\tau_{\kappa}\right)\right)^{-1} \mathcal{G}\left(\tau_{\kappa+1}\right) \mathcal{R}\left(\tau_{\kappa+1}, \tau_{\kappa}\right)^{\prime} \\
\mathcal{G}\left(\tau_{\iota f}\right)=-2 \nu(T) e e^{\prime}
\end{array}\right.
$$

$\mathrm{e}$

$$
\left\{\begin{array}{l}
\mathcal{S}\left(\tau_{\kappa}\right)=-\ell\left(\tau_{\kappa}\right) e+\mathcal{R}\left(\tau_{\kappa+1}, \tau_{\kappa}\right)\left(I+\frac{1}{2} \mathcal{G}\left(\tau_{\kappa+1}\right) \mathcal{B}\left(\tau_{\kappa}\right)\right)^{-1} \mathcal{S}\left(\tau_{\kappa+1}\right) \\
\mathcal{S}\left(\tau_{\iota f}\right)=-\ell(T) e
\end{array}\right.
$$

onde se assume que a inversa de $\left(I+\frac{1}{2} \mathcal{G}\left(\tau_{\kappa+1}\right) \mathcal{B}\left(\tau_{\kappa}\right)\right)$ existe para todo $\kappa=\iota_{f}-1, \ldots, 0$.

Proposição 4.2 Suponha que a lei de controle u definida em (4.5) é aplicada à equação (3.39). Se (4.31) é satisfeita para cada $\kappa=\iota_{f}, \ldots, 1$, então:

$$
Z\left(\tau_{\kappa}\right)=\mathcal{S}\left(\tau_{\kappa}\right)+\mathcal{G}\left(\tau_{\kappa}\right) q\left(\tau_{\kappa}\right)
$$

Prova. Por indução, para $\kappa=\iota_{f}$ tem-se que $Z(T)=-\lambda(T) e=-\ell(T) e+2 \nu(T) e e^{\prime} q(T)$, atendendo à proposição. Suponha que a proposição seja satisfeita para $\kappa+1$. De (4.24) e pela hipótese da indução, tem-se que

$$
q\left(\tau_{\kappa+1}\right)=\mathcal{R}\left(\tau_{\kappa+1}, \tau_{\kappa}\right)^{\prime} q\left(\tau_{\kappa}\right)-\frac{1}{2} \mathcal{B}\left(\tau_{\kappa}\right)\left(\mathcal{S}\left(\tau_{\kappa+1}\right)+\mathcal{G}\left(\tau_{\kappa+1}\right) q\left(\tau_{\kappa+1}\right)\right)
$$


Resolvendo (4.37) para $q\left(\tau_{\kappa+1}\right)$, segue que:

$$
\begin{aligned}
q\left(\tau_{\kappa+1}\right)= & \left(I+\frac{1}{2} \mathcal{B}\left(\tau_{\kappa}\right) \mathcal{G}\left(\tau_{\kappa+1}\right)\right)^{-1} \mathcal{R}\left(\tau_{\kappa+1}, \tau_{\kappa}\right)^{\prime} q\left(\tau_{\kappa}\right) \\
& -\frac{1}{2}\left(I+\frac{1}{2} \mathcal{B}\left(\tau_{\kappa}\right) \mathcal{G}\left(\tau_{\kappa+1}\right)\right)^{-1} \mathcal{B}\left(\tau_{\kappa}\right) \mathcal{S}\left(\tau_{\kappa+1}\right)
\end{aligned}
$$

Utilizando (4.31) e (4.36) em (4.27), resulta que:

$$
\begin{aligned}
Z\left(\tau_{\kappa}\right)= & -\left(\ell\left(\tau_{\kappa}\right)+2 \nu\left(\tau_{\kappa}\right) e^{\prime} q\left(\tau_{\kappa}\right)\right) e \\
& +\mathcal{R}\left(\tau_{\kappa+1}, \tau_{\kappa}\right)\left(\mathcal{S}\left(\tau_{\kappa+1}\right)+\mathcal{G}\left(\tau_{\kappa+1}\right) q\left(\tau_{\kappa+1}\right)\right)
\end{aligned}
$$

Substituindo (4.38) em (4.39), obtém-se:

$$
\begin{aligned}
Z\left(\tau_{\kappa}\right)= & -\left(\ell\left(\tau_{\kappa}\right)+2 \nu\left(\tau_{\kappa}\right) e^{\prime} q\left(\tau_{\kappa}\right)\right) e+\mathcal{R}\left(\tau_{\kappa+1}, \tau_{\kappa}\right) \mathcal{S}\left(\tau_{\kappa+1}\right) \\
+ & \mathcal{R}\left(\tau_{\kappa+1}, \tau_{\kappa}\right) \mathcal{G}\left(\tau_{\kappa+1}\right)\left(I+\frac{1}{2} \mathcal{B}\left(\tau_{\kappa}\right) \mathcal{G}\left(\tau_{\kappa+1}\right)\right)^{-1} \mathcal{R}\left(\tau_{\kappa+1}, \tau_{\kappa}\right)^{\prime} q\left(\tau_{\kappa}\right) \\
& -\frac{1}{2} \mathcal{R}\left(\tau_{\kappa+1}, \tau_{\kappa}\right) \mathcal{G}\left(\tau_{\kappa+1}\right)\left(I+\frac{1}{2} \mathcal{B}\left(\tau_{\kappa}\right) \mathcal{G}\left(\tau_{\kappa+1}\right)\right)^{-1} \mathcal{B}\left(\tau_{\kappa}\right) \mathcal{S}\left(\tau_{\kappa+1}\right) \\
= & -\ell\left(\tau_{\kappa}\right) e+\mathcal{R}\left(\tau_{\kappa+1}, \tau_{\kappa}\right) \mathcal{S}\left(\tau_{\kappa+1}\right)-\frac{1}{2} \mathcal{R}\left(\tau_{\kappa+1}, \tau_{\kappa}\right) \mathcal{G}\left(\tau_{\kappa+1}\right) \\
& \cdot\left(I+\frac{1}{2} \mathcal{B}\left(\tau_{\kappa}\right) \mathcal{G}\left(\tau_{\kappa+1}\right)\right)^{-1} \mathcal{B}\left(\tau_{\kappa}\right) \mathcal{S}\left(\tau_{\kappa+1}\right)-2 \nu\left(\tau_{\kappa}\right) e^{\prime} q\left(\tau_{\kappa}\right) e \\
& +\mathcal{R}\left(\tau_{\kappa+1}, \tau_{\kappa}\right) \mathcal{G}\left(\tau_{\kappa+1}\right)\left(I+\frac{1}{2} \mathcal{B}\left(\tau_{\kappa}\right) \mathcal{G}\left(\tau_{\kappa+1}\right)\right)^{-1} \mathcal{R}\left(\tau_{\kappa+1}, \tau_{\kappa}\right)^{\prime} q\left(\tau_{\kappa}\right) .
\end{aligned}
$$

Utilizando o lema da matriz inversa, as seguintes identidades são verificadas:

$$
\left(I+\frac{1}{2} \mathcal{G}\left(\tau_{\kappa+1}\right) \mathcal{B}\left(\tau_{\kappa}\right)\right)^{-1} \mathcal{G}\left(\tau_{\kappa+1}\right)=\mathcal{G}\left(\tau_{\kappa+1}\right)\left(I+\frac{1}{2} \mathcal{B}\left(\tau_{\kappa}\right) \mathcal{G}\left(\tau_{\kappa+1}\right)\right)^{-1}
$$

$\mathrm{e}$

$$
I-\frac{1}{2} \mathcal{G}\left(\tau_{\kappa+1}\right)\left(I+\frac{1}{2} \mathcal{B}\left(\tau_{\kappa}\right) \mathcal{G}\left(\tau_{\kappa+1}\right)\right)^{-1} \mathcal{B}\left(\tau_{\kappa}\right)=\left(I+\frac{1}{2} \mathcal{G}\left(\tau_{\kappa+1}\right) \mathcal{B}\left(\tau_{\kappa}\right)\right)^{-1}
$$


Substituindo estas identidades em (4.40) e utilizando (4.34) e (4.35), encontra-se (4.36), que é o resultado desejado.

Seja para $\kappa=0, \ldots, \iota_{f}-1$,

$$
\begin{aligned}
\mathbb{A}\left(\tau_{\kappa}\right) & =\left(I+\frac{1}{2} \mathcal{B}\left(\tau_{\kappa}\right) \mathcal{G}\left(\tau_{\kappa+1}\right)\right)^{-1} \mathcal{R}\left(\tau_{\kappa+1}, \tau_{\kappa}\right)^{\prime}, \\
\mathbb{D}\left(\tau_{\kappa}\right) & =\frac{1}{2}\left(I+\frac{1}{2} \mathcal{B}\left(\tau_{\kappa}\right) \mathcal{G}\left(\tau_{\kappa+1}\right)\right)^{-1} \mathcal{B}\left(\tau_{\kappa}\right), \\
q^{*}\left(\tau_{\kappa+1}\right) & =\mathbb{A}\left(\tau_{\kappa}\right) q^{*}\left(\tau_{\kappa}\right)-\mathbb{D}\left(\tau_{\kappa}\right) \mathcal{S}\left(\tau_{\kappa+1}\right), q^{*}(0)=q(0) .
\end{aligned}
$$

Finalmente, apresenta-se o seguinte teorema com uma condição necessária de otimalidade para o problema $P G M V(\rho, \ell, \nu)$.

Teorema 4.3 Se a lei de controle ótima u é tal que $u \in \Pi(P G M V(\rho, \ell, \nu))$, então u é descrito por (4.5), com $\lambda\left(\tau_{\kappa}\right)=\ell\left(\tau_{\kappa}\right)+2 \nu\left(\tau_{\kappa}\right) e^{\prime} q^{*}\left(\tau_{\kappa}\right)$ em (4.2) e $q^{*}\left(\tau_{\kappa}\right)$ dado por (4.43).

Prova. Suponha que $u \in \Pi(P G M V(\rho, \ell, \nu))$. Considerando a Proposição $4.1 \mathrm{e}$ de (4.31), resta apenas mostrar que $E\left(V^{u}\left(\tau_{\kappa}\right)\right)=e^{\prime} q^{*}\left(\tau_{\kappa}\right)$ para cada $\kappa=0, \ldots, \iota_{f}$. Recorda-se que $q_{i}(t)=E\left(V^{u}(t) 1_{\{\theta(t)=i\}}\right)$ e $q(t)=\left(q_{1}(t), \ldots, q_{m}(t)\right)^{\prime}$. Demonstra-se por indução em $\kappa$ que $q\left(\tau_{\kappa}\right)=q^{*}\left(\tau_{\kappa}\right)$. Para $\kappa=0$ o resultado segue pela definição em (4.43). Supondo que a igualdade é válida para $\kappa$, ou seja, $q\left(\tau_{\kappa}\right)=q^{*}\left(\tau_{\kappa}\right)$. Da Proposição 4.2, das equações (4.38) e (4.43), e da hipótese de indução, segue que $q\left(\tau_{\kappa+1}\right)=\mathbb{A}\left(\tau_{\kappa}\right) q^{*}\left(\tau_{\kappa}\right)-\mathbb{D}\left(\tau_{\kappa}\right) \mathcal{S}\left(\tau_{\kappa+1}\right)=q^{*}\left(\tau_{\kappa+1}\right)$, chegando, portanto, ao resultado desejado, já que $E\left(V^{u}\left(\tau_{\kappa}\right)\right)=e^{\prime} q\left(\tau_{\kappa}\right)$.

A partir do Teorema 4.3, descreve-se o seguinte algoritmo de condição necessária para se determinar uma estratégia ótima de controle para o problema generalizado de otimização de portfólio em média-variância com saltos Markovianos:

Passo i Calcular recursivamente as matrizes $\mathcal{G}\left(\tau_{\kappa}\right)$ e vetores $\mathcal{S}\left(\tau_{\kappa}\right)$ como em (4.34) e (4.35), respectivamente, para $\kappa=\iota_{f}, \ldots, 0$; 
Passo ii Calcular recursivamente $q^{*}\left(\tau_{\kappa}\right)$ dado por (4.43) para $\kappa=0, \ldots, \iota_{f}$;

Passo iii Fixar $\lambda\left(\tau_{\kappa}\right)=\ell\left(\tau_{\kappa}\right)+2 \nu\left(\tau_{\kappa}\right) e^{\prime} q^{*}\left(\tau_{\kappa}\right)$ para $\kappa=0, \ldots, \iota_{f}$, e $\lambda(k)=0$, para $k \notin \mathcal{I}$

Passo iv Calcular recursivamente $K(t)$ e $Z(t)$ a partir de (4.2), para $t=T, \ldots, 0$;

Passo v A estratégia ótima de investimento é então obtida por (4.5).

\subsubsection{Condição Suficiente}

Nesta subseção, deriva-se uma condição suficiente para a existência de uma solução $(u)$ para o problema $P G M V(\rho, \ell, \nu)$, tal que $u \in \Pi(P G M V(\rho, \ell, \nu))$. Para isto, demonstrase que é suficiente identificar uma lei de controle ótima para (3.32) que se atenda à expressão:

$$
u^{M}(t)=-\phi_{\theta(t)}(t)^{-1} \varphi_{\theta(t)}(t) V(t)-\frac{\mathcal{E}_{\theta(t)}[M(t+1)]}{2 \mathcal{E}_{\theta(t)}[K(t+1)]} \phi_{\theta(t)}(t)^{-1} \chi_{\theta(t)}(t)
$$

onde

$$
M=(M(1), \ldots, M(T)), M(t) \in \mathbb{R}^{m} .
$$

Denota-se por $\mathcal{U}^{*} \subset \mathcal{U}$ o conjunto de controles ou estratégias admissíveis $u^{M}$ escritas como em (4.44) para algum $M$. A partir de (3.32), para qualquer $u \in \mathcal{U}$, define-se que

$$
\mathcal{J}(u)=\sum_{t \in \mathcal{I}}\left(\rho(t) E\left(V^{u}(t)^{2}\right)-\ell(t) E\left(V^{u}(t)\right)-\nu(t) E\left(V^{u}(t)\right)^{2}\right)
$$

Proposição 4.3 Se $\hat{u}^{M} \in \mathcal{U}^{*}$ é tal que $\mathcal{J}\left(\hat{u}^{M}\right)=\min _{u^{M} \in \mathcal{U}^{*}} \mathcal{J}\left(u^{M}\right)$ então:

$$
\hat{u}^{M} \in \Pi(P G M V(\rho, \ell, \nu)) .
$$

Prova. Demonstra-se a seguir que para qualquer $u \in \mathcal{U}$ é possível encontrar $M$ como em (4.45), tal que $u^{M} \in \mathcal{U}^{*}$ e $\mathcal{J}\left(u^{M}\right) \leq \mathcal{J}(u)$ 
Tomando $M(t)=Z(t)$, com $Z(t)$ como em (4.2), com $\lambda(t)=\ell(t)+2 \nu(t) E\left(V^{u}(t)\right)$, para $t \in \mathcal{I}$, e $\lambda(t)=0$ para $t \notin \mathcal{I}$, e definindo $u^{M}$ como em (4.44), tem-se pelo fato de que $u^{M} \in \Pi(A(\lambda, \rho))$ que para qualquer $u \in \mathcal{U}$, então

$$
\sum_{t \in \mathcal{I}}\left(\rho(t) E\left(V^{u^{M}}(t)^{2}\right)-\lambda(t) E\left(V^{u^{M}}(t)\right)\right) \leq \sum_{t \in \mathcal{I}}\left(\rho(t) E\left(V^{u}(t)^{2}\right)-\lambda(t) E\left(V^{u}(t)\right)\right) .
$$

Utilizando o controle $u^{M}$ e (4.31) em (4.46), encontra-se que

$$
\begin{aligned}
\mathcal{J}\left(u^{M}\right)= & \sum_{t \in \mathcal{I}}\left(\rho(t) E\left(V^{u^{M}}(t)^{2}\right)-\ell(t) E\left(V^{u^{M}}(t)\right)-\nu(t) E\left(V^{u^{M}}(t)\right)^{2}\right) \\
= & \sum_{t \in \mathcal{I}}\left(\rho(t) E\left(V^{u^{M}}(t)^{2}\right)-\left(\lambda(t)-2 \nu(t) E\left(V^{u}(t)\right)\right)\right. \\
& \left.\cdot E\left(V^{u^{M}}(t)\right)-\nu(t) E\left(V^{u^{M}}(t)\right)^{2}\right) \\
= & \sum_{t \in \mathcal{I}}\left(\left(\rho(t) E\left(V^{u^{M}}(t)^{2}\right)-\lambda(t) E\left(V^{u^{M}}(t)\right)\right)\right. \\
& \left.-\nu(t)\left(E\left(V^{u^{M}}(t)\right)^{2}-2 E\left(V^{u}(t)\right) E\left(V^{u^{M}}(t)\right)\right)\right)
\end{aligned}
$$

e

$$
\begin{aligned}
\mathcal{J}(u) & =\sum_{t \in \mathcal{I}}\left(\rho(t) E\left(V^{u}(t)^{2}\right)-\left(\lambda(t)-2 \nu(t) E\left(V^{u}(t)\right)\right) E\left(V^{u}(t)\right)-\nu(t) E\left(V^{u}(t)\right)^{2}\right) \\
& =\sum_{t \in \mathcal{I}}\left(\left(\rho(t) E\left(V^{u}(t)^{2}\right)-\lambda(t) E\left(V^{u}(t)\right)\right)+\nu(t) E\left(V^{u}(t)\right)^{2}\right)
\end{aligned}
$$

De (4.47), (4.48) e (4.49), obtém-se

$$
\mathcal{J}\left(u^{M}\right) \leq \mathcal{J}\left(u^{M}\right)+\sum_{t \in \mathcal{I}} \nu(t) E\left(V^{u}(t)-V^{u^{M}}(t)\right)^{2} \leq \mathcal{J}(u)
$$

demonstrando o resultado desejado.

A partir da Proposição 4.3 e das equações (4.12), (4.13), (4.22) e (4.23) é possível 
reescrever o problema estocástico $P G M V(\rho, \ell, \nu)$ na forma determinística como segue:

$$
\min _{M} \sum_{t \in \mathcal{I}}\left(\rho(t) e^{\prime} g^{M}(t)-\ell(t) e^{\prime} q^{M}(t)-\nu(t)\left(e^{\prime} q^{M}(t)\right)^{2}\right)
$$

com

$$
\begin{aligned}
& q^{M}(t+1)=\mathcal{R}(t+1, t)^{\prime} q^{M}(t)-h(M, K, t), \\
& g^{M}(t+1)=\mathcal{Q}(t+1, t)^{\prime} g^{M}(t)+r(M, K, t),
\end{aligned}
$$

onde $M$, como em (4.45) é a variável de controle. Através da programação dinâmica, encontra-se a solução do problema (4.50). Para isto, define-se, para $k=T-1, \ldots, 0$, os seguintes problemas intermediários

$$
\mathcal{V}\left(g_{k}, q_{k}, k\right)=\min _{M^{k}} \sum_{t \in \mathcal{I}}\left(\rho(t) e^{\prime} g^{M^{k}}(t)-\ell(t) e^{\prime} q^{M^{k}}(t)-\nu(t)\left(e^{\prime} q^{M^{k}}(t)\right)^{2}\right)
$$

onde $M^{k}=(M(k+1), \ldots, M(T)), M(t) \in \mathbb{R}^{m}$, e $g^{M^{k}}(t)$ e $q^{M^{k}}(t)$ são descritos por (4.51) e (4.52) com condição inicial $g(k)=g_{k}$ e $q(k)=q_{k}$, respectivamente.

No que segue, recorda-se que (ver, por exemplo, Saberi et al. (1995), páginas 1213)) para qualquer matriz $S \in \mathbb{R}^{m \times m}$, a inversa generalizada de $S$ (ou inversa MoorePenrose de $S$ ), denotada por $S^{\dagger} \in \mathbb{R}^{m \times m}$ é tal que: i) $S S^{\dagger} S=S$, ii) $S^{\dagger} S S^{\dagger}=S^{\dagger}$, iii) $\left(S S^{\dagger}\right)^{\prime}=S S^{\dagger}$, e iv) $\left(S^{\dagger} S\right)^{\prime}=S^{\dagger} S$. Denota-se ainda por $\operatorname{Im}(S)$ a imagem da matriz $S \in \mathbb{R}^{m \times m}$.

Define-se recursivamente para $k=T-1, \ldots, 0$ as matrizes simétricas $m$-dimensionais $\Psi(k), \Lambda(k)$, o vetor $m$-dimensional $L(k)$ e os números reais $\varepsilon(k)$ como segue:

$$
\Psi(k)=(I+\mathrm{B}(k) \Lambda(k+1)) \mathrm{B}(k),
$$




$$
\begin{aligned}
\Lambda(k)= & -\nu(k) e e^{\prime}+\left(\mathcal{R}(k+1, k)\left(I-\Lambda(k+1) \mathrm{B}(k) \Psi(k)^{\dagger} \mathrm{B}(k)\right)\right) \\
& \cdot \Lambda(k+1) \mathcal{R}(k+1, k)^{\prime}, \\
\Lambda(T)= & -\nu(T) e e^{\prime} \\
L(k)= & -\ell(k) e+\mathcal{R}(k+1, k)\left(I-\Lambda(k+1) \mathrm{B}(k) \Psi(k)^{\dagger} \mathrm{B}(k)\right) L(k+1), \\
L(T)= & -\ell(T) e \\
\varepsilon(k)= & -\frac{1}{4} L(k+1)^{\prime} \mathrm{B}(k) \Psi(k)^{\dagger} \mathrm{B}(k) L(k+1)+\varepsilon(k+1), \\
\varepsilon(T)= & 0,
\end{aligned}
$$

com $\mathrm{B}(k) \geq 0$ dado por (4.9).

O seguinte Teorema estabelece uma condição suficiente para a existência de uma solução $u$, tal que $u \in \Pi(P G M V(\rho, \ell, \nu))$.

Teorema 4.4 Se para cada $k=T-1, \ldots, 0$, tem-se que $\Psi(k) \geq 0$ e, para qualquer $q \in \mathbb{R}^{m}$,

$$
\mathrm{B}(k)\left(\frac{1}{2} L(k+1)+\Lambda(k+1) \mathcal{R}(k+1, k)^{\prime} q\right) \in \operatorname{Im}(\Psi(k)),
$$

então

$$
\mathcal{V}\left(g_{k}, q_{k}, k\right)=K(k)^{\prime} g_{k}+L(k)^{\prime} q_{k}+q_{k}^{\prime} \Lambda(k) q_{k}+\varepsilon(k)
$$

e uma solução ótima para o problema (4.50) é dada, para $k=0, \ldots, T-1$, por

$$
M^{*}(k+1)=\Psi(k)^{\dagger} \mathrm{B}(k)\left(L(k+1)+2 \Lambda(k+1) \mathcal{R}(k+1, k)^{\prime} q(k)\right) .
$$

Prova. Por indução, tem-se de (4.56), (4.58), (4.60) que (4.62) é verdadeira para $k=T$. Suponha que (4.62) vale para $k+1$. Recorda-se de (4.22) e (4.23) que para $\mathbf{M} \in \mathbb{R}^{m}$,

$$
q^{\mathbf{M}}(k+1)=\mathcal{R}(k+1, k)^{\prime} q^{\mathbf{M}}(k)-h(\mathbf{M}, K, k)
$$


$\mathrm{e}$

$$
g^{\mathbf{M}}(k+1)=\mathcal{Q}(k+1, k)^{\prime} g^{\mathbf{M}}(k)+r(\mathbf{M}, K, k),
$$

onde se estendeu a definição em (4.20) e (4.21) para $\mathbf{M} \in \mathbb{R}^{m}$ como

$$
h_{j}(\mathbf{M}, K, k)=\frac{1}{2} \sum_{i=1}^{m} p_{i j}(k) \pi_{i}(k) \frac{\mathcal{E}_{i}[\mathbf{M}]}{\mathcal{E}_{i}[K(k+1)]} \beta_{i}(k)
$$

$\mathrm{e}$

$$
r_{j}(\mathbf{M}, K, k)=\frac{1}{4} \sum_{i=1}^{m} p_{i j}(k) \pi_{i}(k)\left(\frac{\mathcal{E}_{i}[\mathbf{M}]}{\mathcal{E}_{i}[K(k+1)]}\right)^{2} \beta_{i}(k),
$$

respectivamente. A partir de (4.9) e (4.66), nota-se a seguinte relação:

$$
h(\mathbf{M}, K, k)=\frac{1}{2} \mathrm{~B}(k) \mathbf{M}
$$

Do princípio de otimalidade de Bellman, tem-se que:

$$
\begin{aligned}
\mathcal{V}\left(g_{k}, q_{k}, k\right)= & \min _{\mathbf{M} \in \mathbb{R}^{m}} \sum_{t \in \mathcal{I}}\left(\rho(t) e^{\prime} g^{\mathbf{M}}(t)-\ell(t) e^{\prime} q^{\mathbf{M}}(t)-\nu(t)\left(e^{\prime} q^{\mathbf{M}}(t)\right)^{2}\right) \\
= & \left(\rho(k) e^{\prime} g_{k}-\ell(t) e^{\prime} q_{k}-\nu(t)\left(e^{\prime} q_{k}\right)^{2}\right) \\
& +\min _{\mathbf{M} \in \mathbb{R}^{m}} \mathcal{V}\left(g^{\mathbf{M}}(k+1), q^{\mathbf{M}}(k+1), k+1\right) .
\end{aligned}
$$

Substituindo (4.62) em (4.69), obtém-se:

$$
\begin{aligned}
\mathcal{V}\left(g_{k}, q_{k}, k\right)= & \left(\rho(k) e^{\prime} g_{k}-\ell(t) e^{\prime} q_{k}-\nu(t)\left(e^{\prime} q_{k}\right)^{2}\right) \\
& +\min _{\mathbf{M} \in \mathbb{R}^{m}}\left[K(k+1)^{\prime} g^{\mathbf{M}}(k+1)+L(k+1)^{\prime} q^{\mathbf{M}}(k+1)\right. \\
& \left.+q^{\mathbf{M}}(k+1)^{\prime} \Lambda(k+1) q^{\mathbf{M}}(k+1)+\varepsilon(k+1)\right] .
\end{aligned}
$$


Utilizando (4.64) e (4.65), encontra-se que:

$$
\begin{aligned}
\mathcal{V}\left(g_{k}, q_{k}, k\right) & =\left(\rho(k) e^{\prime} g_{k}-\ell(t) e^{\prime} q_{k}-\nu(t) q_{k}^{\prime} e e^{\prime} q_{k}\right) \\
& +\min _{\mathbf{M} \in \mathbb{R}^{m}}\left[K(k+1)^{\prime}\left(\mathcal{Q}(k+1, k)^{\prime} g_{k}+r(\mathbf{M}, K, k)\right)\right. \\
& +L(k+1)^{\prime}\left(\mathcal{R}(k+1, k)^{\prime} q_{k}-h(\mathbf{M}, K, k)\right)+\left(\mathcal{R}(k+1, k)^{\prime} q_{k}-h(\mathbf{M}, K, k)\right)^{\prime} \\
& \left.\cdot \Lambda(k+1)\left(\mathcal{R}(k+1, k)^{\prime} q_{k}-h(\mathbf{M}, K, k)\right)+\varepsilon(k+1)\right] \\
& =\left(\rho(k) e^{\prime} g_{k}-\ell(t) e^{\prime} q_{k}-\nu(t) q_{k}^{\prime} e e^{\prime} q_{k}\right)+K(k+1)^{\prime} \mathcal{Q}(k+1, k)^{\prime} g_{k} \\
& +L(k+1)^{\prime} \mathcal{R}(k+1, k)^{\prime} q_{k}+q_{k}^{\prime} \mathcal{R}(k+1, k) \Lambda(k+1) \mathcal{R}(k+1, k)^{\prime} q_{k} \\
& +\varepsilon(k+1)+\min _{\mathbf{M} \in \mathbb{R}^{m}}\left[K(k+1)^{\prime} r(\mathbf{M}, K, k)-L(k+1)^{\prime} h(\mathbf{M}, K, k)\right. \\
& -q_{k}^{\prime} \mathcal{R}(k+1, k) \Lambda(k+1) h(\mathbf{M}, K, k)-h(\mathbf{M}, K, k)^{\prime} \Lambda(k+1) \mathcal{R}(k+1, k)^{\prime} q_{k} \\
& +h(\mathbf{M}, K, k)^{\prime} \Lambda(k+1) h(\mathbf{M}, K, k) .
\end{aligned}
$$

Substituindo a relação (4.68) acima, obtém-se

$$
\begin{aligned}
\mathcal{V}\left(g_{k}, q_{k}, k\right)= & \rho(k) e^{\prime} g_{k}-\ell(k) e^{\prime} q_{k}-\nu(k) q_{k}^{\prime} e e^{\prime} q_{k}+K(k+1)^{\prime} \mathcal{Q}(k+1, k)^{\prime} g_{k} \\
& +L(k+1)^{\prime} \mathcal{R}(k+1, k)^{\prime} q_{k}+q_{k}^{\prime} \mathcal{R}(k+1, k) \Lambda(k+1) \mathcal{R}(k+1, k)^{\prime} q_{k} \\
& +\varepsilon(k+1)+\min _{\mathbf{M} \in \mathbb{R}^{m}}\left[K(k+1)^{\prime} r(\mathbf{M}, K, k)-L(k+1)^{\prime} h(\mathbf{M}, K, k)\right. \\
& \left.-\mathbf{M}^{\prime} \mathrm{B}(k) \Lambda(k+1) \mathcal{R}(k+1, k)^{\prime} q_{k}+\frac{1}{4} \mathbf{M}^{\prime} \mathrm{B}(k) \Lambda(k+1) \mathrm{B}(k) \mathbf{M}\right] .
\end{aligned}
$$

Observando que $K(k+1)^{\prime} r(\mathbf{M}, K, k)=\frac{1}{4} \mathbf{M}^{\prime} \mathrm{B}(k) \mathbf{M}$ e que $L(k+1)^{\prime} h(\mathbf{M}, K, k)=$ $\frac{1}{2} \mathbf{M}^{\prime} \mathrm{B}(k)^{\prime} L(k+1)$, reescreve-se (4.70) como: 


$$
\begin{aligned}
\mathcal{V}\left(g_{k}, q_{k}, k\right)= & \rho(k) e^{\prime} g_{k}-\ell(k) e^{\prime} q_{k}-\nu(k) q_{k}^{\prime} e e^{\prime} q_{k}+K(k+1)^{\prime} \mathcal{Q}(k+1, k)^{\prime} g_{k} \\
& +L(k+1)^{\prime} \mathcal{R}(k+1, k)^{\prime} q_{k}+q_{k}^{\prime} \mathcal{R}(k+1, k) \Lambda(k+1) \mathcal{R}(k+1, k)^{\prime} q_{k} \\
& +\varepsilon(k+1)+\min _{\mathbf{M} \in \mathbb{R}^{m}}\left[\frac{1}{4} \mathbf{M}^{\prime}\left(\mathrm{B}(k)+\mathrm{B}(k)^{\prime} \Lambda(k+1) \mathrm{B}(k)\right) \mathbf{M}\right. \\
& \left.-\mathbf{M}^{\prime} \mathrm{B}(k)\left(\frac{1}{2} L(k+1)+\Lambda(k+1) \mathcal{R}(k+1, k)^{\prime} q_{k}\right)\right]
\end{aligned}
$$

A partir do Lema 4.2 em (Rami et al. (2002)), tem-se que se (4.61) é válido, então

$$
\begin{aligned}
& \mathrm{B}(k)\left(\frac{1}{2} L(k+1)+\Lambda(k+1) \mathcal{R}(k+1, k)^{\prime} q(k)\right)= \\
& \Psi(k)^{\dagger} \Psi(k) \mathrm{B}(k)\left(\frac{1}{2} L(k+1)+\Lambda(k+1) \mathcal{R}(k+1, k)^{\prime} q(k)\right)
\end{aligned}
$$

e, portanto, segue que

$$
\begin{aligned}
& \frac{1}{4} \mathbf{M}^{\prime} \Psi(k) \mathbf{M}-\mathbf{M}^{\prime} \mathrm{B}(k)\left(\frac{1}{2} L(k+1)+\Lambda(k+1) \mathcal{R}(k+1, k)^{\prime} q_{k}\right)= \\
& \frac{1}{4}\left(\left(\mathbf{M}-M^{*}(k+1)\right)^{\prime} \Psi(k)\left(\mathbf{M}-M^{*}(k+1)\right)-M^{*}(k+1)^{\prime} \Psi(k) M^{*}(k+1)\right) .
\end{aligned}
$$

Se $\Psi(k) \geq 0$, então é fácil verificar de (4.72) que o mínimo em (4.71) é atingido para $\mathbf{M}=M^{*}(k+1)$. Assim, fazendo $\mathbf{M}=M^{*}(k+1)$, como em (4.63), e substituindo em (4.71), verifica-se que:

$$
\begin{aligned}
\mathcal{V}\left(g_{k}, q_{k}, k\right)= & \rho(k) e^{\prime} g_{k}-\ell(k) e^{\prime} q_{k}-\nu(k) q_{k}^{\prime} e e^{\prime} q_{k}+K(k+1)^{\prime} \mathcal{Q}(k+1, k)^{\prime} g_{k} \\
& +L(k+1)^{\prime} \mathcal{R}(k+1, k)^{\prime} q_{k}+q_{k}^{\prime} \mathcal{R}(k+1, k) \Lambda(k+1) \mathcal{R}(k+1, k)^{\prime} q_{k} \\
& +\varepsilon(k+1)+\frac{1}{4}\left(\Psi(k)^{-1} \mathrm{~B}(k)\left(L(k+1)+2 \Lambda(k+1) \mathcal{R}(k+1, k)^{\prime} q_{k}\right)\right)^{\prime} \\
& \cdot \Psi(k) \Psi(k)^{-1} \mathrm{~B}(k)\left(L(k+1)+2 \Lambda(k+1) \mathcal{R}(k+1, k)^{\prime} q_{k}\right) \\
& -\left(\Psi(k)^{-1} \mathrm{~B}(k)\left(L(k+1)+2 \Lambda(k+1) \mathcal{R}(k+1, k)^{\prime} q_{k}\right)\right)^{\prime} \\
& \cdot \frac{1}{2} \mathrm{~B}(k)\left(L(k+1)+2 \Lambda(k+1) \mathcal{R}(k+1, k)^{\prime} q_{k}\right),
\end{aligned}
$$


$\mathrm{e}$

$$
\begin{aligned}
& \mathcal{V}\left(g_{k}, q_{k}, k\right)=\rho(k) e^{\prime} g_{k}-\ell(k) e^{\prime} q_{k}-\nu(k) q_{k}^{\prime} e e^{\prime} q_{k}+K(k+1)^{\prime} \mathcal{Q}(k+1, k)^{\prime} g_{k} \\
& +L(k+1)^{\prime} \mathcal{R}(k+1, k)^{\prime} q_{k}+q_{k}^{\prime} \mathcal{R}(k+1, k) \Lambda(k+1) \mathcal{R}(k+1, k)^{\prime} q_{k} \\
& +\varepsilon(k+1)+\frac{1}{4}\left(L(k+1)^{\prime}+2 q_{k}^{\prime} \mathcal{R}(k+1, k) \Lambda(k+1)^{\prime}\right) \mathrm{B}(k) \Psi(k)^{-1} \\
& \cdot \mathrm{B}(k)\left(L(k+1)+2 \Lambda(k+1) \mathcal{R}(k+1, k)^{\prime} q_{k}\right) \\
& -\left(L(k+1)^{\prime}+2 q_{k}^{\prime} \mathcal{R}(k+1, k) \Lambda(k+1)^{\prime}\right) \mathrm{B}(k) \Psi(k)^{-1} \\
& \cdot \frac{1}{2} \mathrm{~B}(k)\left(L(k+1)+2 \Lambda(k+1) \mathcal{R}(k+1, k)^{\prime} q_{k}\right) \\
& =\rho(k) e^{\prime} g_{k}-\ell(k) e^{\prime} q_{k}-\nu(k) q_{k}^{\prime} e e^{\prime} q_{k}+K(k+1)^{\prime} \mathcal{Q}(k+1, k)^{\prime} g_{k} \\
& +L(k+1)^{\prime} \mathcal{R}(k+1, k)^{\prime} q_{k}+q_{k}^{\prime} \mathcal{R}(k+1, k) \Lambda(k+1) \mathcal{R}(k+1, k)^{\prime} q_{k} \\
& +\varepsilon(k+1)+\left(L(k+1)^{\prime}+2 q_{k}^{\prime} \mathcal{R}(k+1, k) \Lambda(k+1)^{\prime}\right) \mathrm{B}(k) \Psi(k)^{-1} \\
& \cdot\left(\frac{1}{4} \mathrm{~B}(k)\left(L(k+1)+2 \Lambda(k+1) \mathcal{R}(k+1, k)^{\prime} q_{k}\right)\right. \\
& \left.-\frac{1}{2} \mathrm{~B}(k)\left(L(k+1)+2 \Lambda(k+1) \mathcal{R}(k+1, k)^{\prime} q_{k}\right)\right) \\
& =\rho(k) e^{\prime} g_{k}-\ell(k) e^{\prime} q_{k}-\nu(k) q_{k}^{\prime} e e^{\prime} q_{k}+K(k+1)^{\prime} \mathcal{Q}(k+1, k)^{\prime} g_{k} \\
& +L(k+1)^{\prime} \mathcal{R}(k+1, k)^{\prime} q_{k}+q_{k}^{\prime} \mathcal{R}(k+1, k) \Lambda(k+1) \mathcal{R}(k+1, k)^{\prime} q_{k} \\
& +\varepsilon(k+1)+\left(L(k+1)^{\prime}+2 q_{k}^{\prime} \mathcal{R}(k+1, k) \Lambda(k+1)^{\prime}\right) \mathrm{B}(k) \Psi(k)^{-1} \\
& \cdot\left(-\frac{1}{4} \mathrm{~B}(k)^{\prime}\left(L(k+1)+2 \Lambda(k+1) \mathcal{R}(k+1, k)^{\prime} q_{k}\right)\right) \\
& =\rho(k) e^{\prime} g_{k}-\ell(k) e^{\prime} q_{k}-\nu(k) q_{k}^{\prime} e e^{\prime} q_{k}+K(k+1)^{\prime} \mathcal{Q}(k+1, k)^{\prime} g_{k} \\
& +L(k+1)^{\prime} \mathcal{R}(k+1, k)^{\prime} q_{k}+q_{k}^{\prime} \mathcal{R}(k+1, k) \Lambda(k+1) \mathcal{R}(k+1, k)^{\prime} q_{k} \\
& +\varepsilon(k+1)-\frac{1}{4} L(k+1)^{\prime} \mathrm{B}(k) \Psi(k)^{-1} \mathrm{~B}(k)^{\prime} L(k+1) \\
& -\frac{1}{2} q_{k}^{\prime} \mathcal{R}(k+1, k) \Lambda(k+1)^{\prime} \mathrm{B}(k) \Psi(k)^{-1} \mathrm{~B}(k)^{\prime} L(k+1) \\
& -\frac{1}{2} L(k+1)^{\prime} \mathrm{B}(k) \Psi(k)^{-1} \mathrm{~B}(k)^{\prime} \Lambda(k+1) \mathcal{R}(k+1, k)^{\prime} q_{k} \\
& -q_{k}^{\prime} \mathcal{R}(k+1, k) \Lambda(k+1)^{\prime} \mathrm{B}(k) \Psi(k)^{-1} \mathrm{~B}(k)^{\prime} \Lambda(k+1) \mathcal{R}(k+1, k)^{\prime} q_{k},
\end{aligned}
$$


rearranjando os termos, encontra-se:

$$
\begin{aligned}
\mathcal{V}\left(g_{k}, q_{k}, k\right)= & \left(K(k+1)^{\prime} \mathcal{Q}(k+1, k)^{\prime}+\rho(k) e^{\prime}\right) g_{k} \\
& +\left(-\ell(k) e^{\prime}+L(k+1)^{\prime}\left(I-\mathrm{B}(k) \Psi(k)^{-1} \mathrm{~B}(k) \Lambda(k+1)\right) \mathcal{R}(k+1, k)^{\prime}\right) q_{k} \\
& +q_{k}^{\prime}\left(\left(\mathcal{R}(k+1, k)\left(I-\Lambda(k+1)^{\prime} \mathrm{B}(k) \Psi(k)^{-1} \mathrm{~B}(k)\right)\right)\right. \\
& \left.\cdot \Lambda(k+1) \mathcal{R}(k+1, k)^{\prime}-\nu(k) e e^{\prime}\right) q_{k} \\
& -\frac{1}{4} L(k+1)^{\prime} \mathrm{B}(k) \Psi(k)^{-1} \mathrm{~B}(k) L(k+1)+\varepsilon(k+1),
\end{aligned}
$$

completando a prova.

Observação 4.1 Se para cada $k=T-1, \ldots, 0$, tem-se que $\Psi(k)>0$, então facilmente se verifica que (4.61) é satisfeita, pois $\Psi(k)^{\dagger}=\Psi(k)^{-1}$, e a solução ótima (4.63) é única. Se $I+\Lambda(k+1) \mathrm{B}(k)$ é não singular, então, como $\Psi(k)=\mathrm{B}(k)(I+\Lambda(k+1) \mathrm{B}(k))$, segue que $\operatorname{Im}(\Psi(k))=\operatorname{Im}(\mathrm{B}(k))$ e claramente (4.61) é satisfeita.

Do Teorema 4.4, elabora-se o seguinte algoritmo de condição suficiente para determinar a estratégia ótima de controle para o problema generalizado de otimização de portfólio em média-variância com saltos:

Passo i Calcular recursivamente as matrizes $\Lambda(k)$ e $\Psi(k)$, e os vetores $L(k)$ como em (4.55)-(4.58) para $k=T-1, \ldots, 0$;

Passo ii Calcular recursivamente $q^{M^{*}}(k)$, dado por (4.51), para a estratégia $M^{*}$, expressa como em (4.63), para $k=0, \ldots, T$;

Passo iii A estratégia ótima é descrita pela expressão (4.44) com $M=M^{*}$.

Observação 4.2 A partir do Teorema 4.3, a solução obtida do algoritmo de condição suficiente deve coincidir com a solução obtida pelo algoritmo de condição necessária apresentado na subseção anterior. 


\section{Capítulo 5}

\section{Aplicação do $P G M V$ a Problemas Específicos de Média-Variância}

Neste capítulo, apresenta-se como utilizar a solução do problema $P G M V(\rho, \ell, \nu)$ para encontrar as soluções para os problemas $P B C(\varrho, \psi), P M V\left(\sigma^{2}\right), P M V(\epsilon), P\left(\sigma^{2}\right)$ e $P(\epsilon)$ introduzidos no Capítulo 3 para o caso geral em que todos os ativos são de risco. Para os problemas $P\left(\sigma^{2}\right)$ e $P(\epsilon)$, deriva-se também uma solução analítica, para o caso em que existe um ativo livre de risco, que não está sujeito a nenhuma fonte de incerteza.

Inicia-se apresentando o processo para encontrar a solução dos problemas em que tanto a função objetivo quanto as restrições incidem em todos os períodos. A seguir, demonstra-se que, quando a restrição incide apenas no período final, é possível derivar uma forma fechada para a solução dos problemas $P M V\left(\sigma^{2}\right), P M V(\epsilon), P\left(\sigma^{2}\right)$ e $P(\epsilon)$. 


\subsection{Solução para os Problemas de Média-Variância com Custo e Controle nos Períodos}

\section{Intermediários}

Nesta seção apresenta-se como determinar as soluções para os problemas $P B C(\varrho, \psi)$, $P M V\left(\sigma^{2}\right)$ e $P M V(\epsilon)$ quando a função objetivo e as restrições incidem em todos os instantes $t \in \mathcal{T}$. Inicia-se com o problema $P B C(\varrho, \psi)$.

A partir da solução do problema generalizado de média-variância apresentado na Seção 4.3, encontra-se a solução para o problema $P B C(\varrho, \psi)$. Como descrito na Seção 3.3 , o problema $P B C(\varrho, \psi)$ é um problema cuja função objetivo é maximizar a soma ponderada do valor esperado da riqueza do investidor ao longo do tempo, com restrições sobre a probabilidade máxima do portfólio cair abaixo de um dado valor nos instantes $t \in \mathcal{T}$.

Recorda-se que o problema $P B C(\varrho, \psi)$ definido como abaixo:

$$
\begin{aligned}
& \operatorname{PBC}(\varrho, \psi) \quad: \max _{u \in \mathcal{U}} \sum_{t \in \mathcal{T}} E(V(t)) \\
& \operatorname{Var}(V(t)) \leq \varrho(t)[E(V(t)-\psi(t))]^{2}, t \in \mathcal{T},
\end{aligned}
$$

pode ser reescrito na formulação sem restrição a seguir

$$
\begin{aligned}
& \operatorname{LPBC}(\omega, \varrho, \psi): \max _{u \in \mathcal{U}} \sum_{t \in \mathcal{T}}(\alpha(t)-2 \omega(t) \varrho(t) \psi(t)) E(V(t))-\sum_{t \in \mathcal{T}} \omega(t) E\left(V(t)^{2}\right) \\
& +\sum_{t \in \mathcal{T}} \omega(t)(1+\varrho(t)) E(V(t))^{2}+\sum_{t \in \mathcal{T}} \omega(t) \varrho(t) \psi(t)^{2}
\end{aligned}
$$

agregando-se para isso os Multiplicadores de Lagrange $\omega(t)$, para $t \in \mathcal{T}$. Dessa forma, foi estabelecida na Subseção 3.3 .1 uma relação entre $\operatorname{LPBC}(\omega, \varrho, \psi)$ e $P G M V(\rho, \ell, \nu)$, 
fazendo, para $t \in \mathcal{I}=\mathcal{T}$,

$$
\left\{\begin{array}{l}
\rho(t)=\omega(t) \\
\nu(t)=\rho(t)(1+\varrho(t)) \\
\ell(t)=\alpha(t)-2 \rho(t) \varrho(t) \psi(t) .
\end{array}\right.
$$

No capítulo anterior, resolvendo o problema $P G M V(\rho, \ell, \nu)$, encontrou-se como resultado expressões para a esperança e para a variância da riqueza do investidor em função de $\rho, \ell$ e $\nu$. Em (5.1), os coeficientes $\ell$ e $\nu$ foram definidos em função de $\rho=\omega$. Portanto, a partir da solução de $\operatorname{PGMV}(\rho, \ell, \nu)$, para resolver o problema $P B C(\varrho, \psi)$ resta apenas encontrar a sequência de números positivos $\omega(t)$, para $t \in \mathcal{T}$, que atendem às restrições em $P B C(\varrho, \psi)$ e produzem a maior soma ponderada das esperanças da riqueza ao longo do tempo $t \in \mathcal{T}$. Devido a sua complexidade, este passo da solução é realizado somente por algoritmo numérico, utilizando-se para isso qualquer pacote de otimização disponível. Alternativamente, Zhu et al. (2004) propuseram um algoritmo primal-dual para realizar esta tarefa.

Analisa-se agora o processo de solução dos problemas $P M V\left(\sigma^{2}\right)$ e $P M V(\epsilon)$ quando a função objetivo e as restrições incidem em todos os períodos. Como descrito na Seção 3.3, o problema $P M V\left(\sigma^{2}\right)$ é um problema multi-período de média-variância no qual a função objetivo é constituída pela soma ponderada dos valores esperados da riqueza do investidor ao longo do tempo, com restrições sobre a variância deste patrimônio em cada instante $t \in \mathcal{T}$. O problema $\operatorname{PMV}(\epsilon)$ se diferencia do $\operatorname{PMV}\left(\sigma^{2}\right)$, pois sua a função objetivo é formada pela soma ponderada das variâncias da riqueza do investidor ao longo do tempo, com restrições sobre o valor esperado deste patrimônio em cada instante $t \in \mathcal{T}$. Para comodidade do leitor, repete-se abaixo as formulações dos problemas $P M V\left(\sigma^{2}\right)$ e $P M V(\epsilon)$ :

$$
\begin{aligned}
P M V\left(\sigma^{2}\right) & : \max _{u \in \mathcal{U}} \sum_{t \in \mathcal{T}} \alpha(t) E(V(t)) \\
\text { sujeito a } & : \quad \operatorname{Var}(V(t)) \leq \sigma^{2}(t), t \in \mathcal{T},
\end{aligned}
$$


e

$$
\begin{aligned}
P M V(\epsilon) & : \max _{u \in \mathcal{U}}-\sum_{t \in \mathcal{T}} \alpha(t) \operatorname{Var}(V(t)) \\
\text { sujeito a } & : \quad E(V(t)) \geq \epsilon(t), t \in \mathcal{T} .
\end{aligned}
$$

Na Seção 3.3, mostrou-se que estes problemas poderiam ser reescritos em uma formulação sem restrição, através da introdução dos Multiplicadores de Lagrange $\omega(t)$, para $t \in \mathcal{T}$, da forma:

$$
\operatorname{LPMV}\left(\omega, \sigma^{2}\right): \max _{u \in \mathcal{U}} \sum_{t \in \mathcal{T}}\left(\omega(t)\left(E(V(k))^{2}-E\left(V(k)^{2}\right)\right)+\alpha(t) E(V(t))\right)
$$

e

$$
\operatorname{LPMV}(\omega, \epsilon): \max _{u \in \mathcal{U}} \sum_{t \in \mathcal{T}}\left(\alpha(t)\left(E(V(k))^{2}-E\left(V(k)^{2}\right)\right)-\omega(t) E(V(t))\right)
$$

podendo estes serem convertidos na formulação generalizada $\operatorname{PGMV}(\rho, \ell, \nu)$, fazendo

$$
\left\{\begin{array}{l}
\rho(t)=\nu(t)=\omega(t), \\
\ell(t)=\alpha(t)
\end{array}\right.
$$

e

$$
\left\{\begin{array}{l}
\rho(t)=\nu(t)=\alpha(t), \\
\ell(t)=-\omega(t),
\end{array}\right.
$$

respectivamente, em $\operatorname{LPMV}\left(\omega, \sigma^{2}\right)$ e $\operatorname{LPMV}(\omega, \epsilon)$.

Assim como descrito acima para o problema $P B C(\varrho, \psi)$, também nestes casos, de posse da solução do problema generalizado, resta apenas encontrar a sequência de números positivos $\omega(t)$, para $t \in \mathcal{T}$, que atendem às restrições e produzem o maior resultado para a respectiva função objetivo. Este passo para solução destes problemas também requer um procedimento numérico. 


\subsection{Solução para os Problemas de Média-Variância com Custo nos Períodos Intermediários e} Restrição apenas no Instante Final

Quando nos problemas $P M V\left(\sigma^{2}\right)$ e $P M V(\epsilon)$ a restrição incide apenas no instante final, mas se permite que a função objetivo atue sobre todos os instantes, uma solução exata pode ser derivada analiticamente. A derivação de formas fechadas para a solução destes problemas é o objetivo desta seção.

Como descrito anteriormente, os problemas $P M V\left(\sigma^{2}\right)$ e $P M V(\epsilon)$ com restrição apenas no instante final são diferentes dos problemas tradicionais de média-variância em multi-período, pois suas funções objetivo são constituídas pela soma ponderada da esperança ou variância da riqueza ao longo do tempo, enquanto os problemas tradicionais consideram na função objetivo apenas o instante final. A vantagem destas formulações sobre as tradicionais é de permitirem que o investidor possa atribuir criteriosamente pesos diferentes a cada período até o horizonte de investimento para serem considerados na função objetivo.

Representa-se por $\Pi\left(P M V\left(\sigma^{2}\right)\right)$ e $\Pi(P M V(\epsilon))$, respectivamente, o conjunto de soluções ótimas dos problemas $P M V\left(\sigma^{2}\right)$ e $P M V(\epsilon)$.

Antes de descrever as soluções dos problemas, convenientemente, define-se que:

$$
\begin{aligned}
a & =q(0)^{\prime} \mathcal{Z}(0), \quad c=g(0)^{\prime} \mathcal{K}(0), \quad b=\sum_{k=0}^{T-1} h(\mathcal{Z}, \mathcal{K}, k)^{\prime} \mathcal{Z}(k+1), \\
c_{0} & =2 \sum_{k=0}^{T-1} h(z, \mathcal{K}, k)^{\prime} z(k+1), \\
c_{1} & =2 \sum_{k=0}^{T-1} h(z, \mathcal{K}, k)^{\prime} \mathcal{Z}(k+1),
\end{aligned}
$$

onde

$$
z(t)=\sum_{s=t}^{T} \ell(s) \mathcal{Z}(s, t) .
$$


Considera-se inicialmente o problema $P M V(\epsilon)$, com restrições apenas no instante final $(T)$. Apresenta-se o seguinte teorema com a solução para este problema.

Teorema 5.1 Seja $\nu(t)=\rho(t)=\alpha(t)$ para $t \in \mathcal{T}$, com $\alpha(T)>0$, e $\ell(t)=\omega(t)=0$ para $t \neq T$. Se $u \in \Pi\left((P G M V(\rho, \ell, \nu))\right.$, com $E\left(V^{u}(T)\right)=\epsilon$, então $u \in \Pi(P M V(\epsilon))$.

Prova. Suponha por contradição que $u \notin \Pi(P M V(\epsilon))$, então para algum $u^{*} \in$ $\Pi(P M V(\epsilon)), \mathrm{com}$

$$
E\left(V^{u^{*}}(T)\right) \geq \epsilon=E\left(V^{u}(T)\right),
$$

tem-se

$$
\sum_{t=1}^{T}-\alpha(t) \operatorname{Var}\left(V^{u^{*}}(t)\right)>\sum_{t=1}^{T}-\alpha(t) \operatorname{Var}\left(V^{u}(t)\right) .
$$

Portanto, de (3.32) e (5.3), resulta que

$$
\sum_{t=1}^{T}\left(-\rho(t) \operatorname{Var}\left(V^{u^{*}}(t)\right)+\ell(t) E\left(V^{u^{*}}(t)\right)\right)>\sum_{t=1}^{T}\left(-\rho(t) \operatorname{Var}\left(V^{u}(t)\right)+\ell(t) E\left(V^{u}(t)\right)\right),
$$

em contradição com o fato de que $u \in \Pi(P G M V(\rho, \ell, \nu))$.

Convém recordar que, para $\kappa=\iota_{f}, \ldots, 0, \mathcal{R}\left(\tau_{\kappa+1}, \tau_{\kappa}\right), \mathcal{B}\left(\tau_{\kappa}\right), \mathcal{G}\left(\tau_{\kappa+1}\right), \mathbb{A}\left(\tau_{\kappa}\right)$ e $\mathbb{D}\left(\tau_{\kappa}\right)$ são como em $(4.4),(4.10),(4.34),(4.41)$ e (4.42).

Corolário 5.1 Se $u \in \Pi(P G M V(\rho, \ell, \nu))$, com $\nu(t)=\rho(t)=\alpha(t)$ para $t \in \mathcal{T}$, com $\alpha(T)>0, \ell(t)=\omega(t)=0$ para $t \neq T$ e com

$$
\ell(T)=\frac{\epsilon-e^{\prime} \prod_{\varsigma=0}^{\iota_{f}-1} \mathbb{A}\left(\tau_{\varsigma}\right) q(0)}{\sum_{\varsigma=0}^{\iota_{f}-1}\left(\prod_{j=\varsigma+1}^{\iota_{f}-1} \mathbb{A}\left(\tau_{j}\right)\right) \mathbb{D}\left(\tau_{\varsigma}\right)\left(\prod_{j=\varsigma}^{\iota_{f}-1} \mathcal{R}\left(\tau_{j+1}, \tau_{j}\right)\left(I+\frac{1}{2} \mathcal{G}\left(\tau_{j+1}\right) \mathcal{B}\left(\tau_{j}\right)\right)^{-1}\right) e}
$$

então $u \in \Pi(P M V(\epsilon))$.

Prova. A prova segue do Teorema 5.1, restando apenas derivar o coeficiente $\ell(T)$. Assim, de (5.3), observa-se que $\nu(t)=\rho(t)=\alpha(t) \geq 0$, onde $\alpha(t)$ é um coeficiente fornecido pelo investidor, para $t \in \mathcal{T}$, e $\ell(t)=\omega(t)=0$ para $t \neq T$. Portanto, a partir 
de (4.34), (4.41) e (4.42), é possível calcular recursivamente $\mathcal{G}\left(\tau_{\kappa}\right), \mathbb{A}\left(\tau_{\kappa}\right)$ e $\mathbb{D}\left(\tau_{\kappa}\right)$ para $\kappa=\iota_{f}, \ldots, 0$. Recorda-se de (4.38), (4.41), (4.42), que

$$
q\left(\tau_{\kappa+1}\right)=\mathbb{A}\left(\tau_{\kappa}\right) q\left(\tau_{\kappa}\right)-\mathbb{D}\left(\tau_{\kappa}\right) \mathcal{S}\left(\tau_{\kappa+1}\right)
$$

De (4.35), é fácil verificar que

$$
\mathcal{S}\left(\tau_{\kappa}\right)=-\ell(T)\left(\prod_{\varsigma=\kappa}^{\iota_{f}-1} \mathcal{R}\left(\tau_{\varsigma+1}, \tau_{\varsigma}\right)\left(I+\frac{1}{2} \mathcal{G}\left(\tau_{\varsigma+1}\right) \mathcal{B}\left(\tau_{\varsigma}\right)\right)^{-1}\right) e .
$$

Logo, detemina-se de (5.9) que

$$
\begin{aligned}
q(T)=\prod_{\varsigma=0}^{\iota_{f}-1} \mathbb{A}\left(\tau_{\varsigma}\right) q(0)+\ell(T) \sum_{\varsigma=0}^{\iota_{f}-1} & \left(\prod_{j=\varsigma+1}^{\iota_{f}-1} \mathbb{A}\left(\tau_{j}\right)\right) \mathbb{D}\left(\tau_{\varsigma}\right) \\
& \cdot\left(\prod_{j=\varsigma}^{\iota_{f}-1} \mathcal{R}\left(\tau_{j+1}, \tau_{j}\right)\left(I+\frac{1}{2} \mathcal{G}\left(\tau_{j+1}\right) \mathcal{B}\left(\tau_{j}\right)\right)^{-1}\right) e .
\end{aligned}
$$

Fazendo $\epsilon=e^{\prime} q(T)$ e resolvendo para $\ell(T)$ encontra-se a expressão desejada.

O teorema a seguir apresenta a solução para o problema $P M V\left(\sigma^{2}\right)$, com restrições apenas no instante final $(T)$.

Teorema 5.2 Seja $\ell(t)=\alpha(t) \geq 0$ para $t \in \mathcal{T}, \operatorname{com} \alpha(T)>0, \nu(t)=\rho(t)=\omega(t)=0$ para $t \neq T, \rho(T)=\nu(T)$. Se $u \in \Pi\left((P G M V(\rho, \ell, \nu))\right.$, com $\operatorname{Var}\left(V^{u}(T)\right)=\sigma^{2}$, então $u \in \Pi\left(P M V\left(\sigma^{2}\right)\right)$.

Prova. Suponha por contradição que $u \notin \Pi\left(P M V\left(\sigma^{2}\right)\right)$, então para algum $u^{*} \in$ $\Pi\left(P M V\left(\sigma^{2}\right)\right), \mathrm{com}$

$$
\operatorname{Var}\left(V^{u^{*}}(T)\right) \leq \sigma^{2}=\operatorname{Var}\left(V^{u}(T)\right)
$$

tem-se

$$
\sum_{t=1}^{T} \alpha(t) E\left(V^{u^{*}}(t)\right)>\sum_{t=1}^{T} \alpha(t) E\left(V^{u}(t)\right)
$$


Logo, de (3.32) e (5.2), obtém-se

$$
\sum_{t=1}^{T}\left(-\rho(t) \operatorname{Var}\left(V^{u^{*}}(t)\right)+\ell(t) E\left(V^{u^{*}}(t)\right)\right)>\sum_{t=1}^{T}\left(-\rho(t) \operatorname{Var}\left(V^{u}(t)\right)+\ell(t) E\left(V^{u}(t)\right)\right)
$$

que contradiz o fato de que $u \in \Pi(P G M V(\rho, \ell, \nu))$.

Corolário 5.2 Se $u \in \Pi(P G M V(\rho, \ell, \nu))$, com $\ell(t)=\alpha(t) \geq 0$ para $t \in \mathcal{T}$, com $\alpha(T)>0, \nu(t)=\rho(t)=\omega(t)=0$ para $t \neq T, \rho(T)=\nu(T)$ e com

$$
\nu(T)=\frac{1}{2} \sqrt{\frac{c_{0}(1-2 b)+c_{1}^{2}}{(1-2 b)\left(\sigma^{2}-c\right)+a^{2}}}
$$

onde $a, b, c, c_{0}$ e $c_{1}$ são dados por (5.4), (5.5) e (5.6), então $u \in \Pi\left(P M V\left(\sigma^{2}\right)\right)$.

Prova. A prova segue do Teorema 5.2, restando apenas derivar o coeficiente $\nu(T)$. Na derivação dos coeficientes para o problema $P M V\left(\sigma^{2}\right)$ é possível utilizar um procedimento mais direto que o utilizado para $P M V(\epsilon)$. Convenientemente, fixa-se, para $u$ como em (4.5), $E\left(V^{u}(T)\right)=v$ e $\eta=2 \nu(T)$. De (5.2), nota-se que $\ell(t)=\alpha(t) \geq 0$ é um coeficiente estipulado pelo investidor, para $t \in \mathcal{T}$ e $\nu(t)=\rho(t)=\omega(t)=0$ para $t \neq T$, de (4.31), resulta que $\lambda(t)=\ell(t)=\alpha(t)$ para $t=0, \ldots, T-1$, e $\lambda(T)=\alpha(T)+\eta v$. Simplifica-se também (4.2), obtendo em $K(t)=\frac{\eta}{2} \mathcal{K}(t)$ e $Z(t)=-(z(t)+\eta v \mathcal{Z}(t))$, onde $z(t)=\sum_{s=t}^{T} \alpha(s) \mathcal{Z}(s, t)$.

Assim, impondo $\operatorname{Var}\left(V^{u}(T)\right)=\sigma^{2}$ e lembrando que $q(T)^{\prime} e=E\left(V^{u}(T)\right)$ e que 
$g(T)^{\prime} e=E\left(V^{u}(T)^{2}\right)$, segue de (5.12), (4.22), (4.23) e (5.4) que:

$$
\begin{aligned}
q(T)^{\prime} e & =q(0)^{\prime} \mathcal{R}(T, 0) e-\sum_{k=0}^{T-1} h(Z, K, k)^{\prime} \mathcal{R}(T, k+1) e \\
v & =q(0)^{\prime} \mathcal{Z}(0)-\sum_{k=0}^{T-1} h\left(-(z+\eta v \mathcal{Z}), \frac{\eta}{2} \mathcal{K}, k\right)^{\prime} \mathcal{Z}(k+1) \\
& =a+\frac{2}{\eta} \sum_{k=0}^{T-1} h(z+\eta v \mathcal{Z}, \mathcal{K}, k)^{\prime} \mathcal{Z}(k+1) \\
& =a+\frac{2}{\eta}\left\{\sum_{k=0}^{T-1} h(z, \mathcal{K}, k)^{\prime} \mathcal{Z}(k+1)+\eta v \sum_{k=0}^{T-1} h(\mathcal{Z}, \mathcal{K}, k)^{\prime} \mathcal{Z}(k+1)\right\} \\
& =a+\frac{2}{\eta} \sum_{k=0}^{T-1} h(z, \mathcal{K}, k)^{\prime} \mathcal{Z}(k+1)+2 v b \\
& =\frac{a \eta+c_{1}}{\eta(1-2 b)}
\end{aligned}
$$

e

$$
\begin{aligned}
g(T)^{\prime} e & =g(0)^{\prime} \mathcal{Q}(T, 0) e+\sum_{k=0}^{T-1} r(Z, K, k)^{\prime} \mathcal{Q}(T, k+1) e \\
\sigma^{2}+v^{2} & =g(0)^{\prime} \mathcal{K}(0)+\sum_{k=0}^{T-1} r\left(-(z+\eta v \mathcal{Z}), \frac{\eta}{2} \mathcal{K}, k\right)^{\prime} \mathcal{K}(k+1) \\
v^{2} & =-\sigma^{2}+c+\frac{2}{\eta^{2}} \sum_{k=0}^{T-1} 2 r(z+\eta v \mathcal{Z}, \mathcal{K}, k)^{\prime} \mathcal{K}(k+1),
\end{aligned}
$$

utilizando a seguinte relação, extraída de (4.20) e (4.21):

$$
\begin{aligned}
2 r(v+p, \mathcal{K}, k)^{\prime} \mathcal{K}(k+1) & =h(v, \mathcal{K}, k)^{\prime} v(k+1) \\
& +h(p, \mathcal{K}, k)^{\prime} p(k+1)+2 h(v, \mathcal{K}, k)^{\prime} p(k+1),
\end{aligned}
$$


resulta que:

$$
\begin{aligned}
v^{2} & =-\sigma^{2}+c+\frac{2}{\eta^{2}}\left\{\sum_{k=0}^{T-1} h(z, \mathcal{K}, k)^{\prime} z(k+1)+(\eta v)^{2} \sum_{k=0}^{T-1} h(\mathcal{Z}, \mathcal{K}, k)^{\prime} \mathcal{Z}(k+1)\right. \\
& \left.+2 \eta v \sum_{k=0}^{T-1} h(z, \mathcal{K}, k)^{\prime} \mathcal{Z}(k+1)\right\} \\
& =-\sigma^{2}+c+\frac{2}{\eta^{2}}\left\{\sum_{k=0}^{T-1} h(z, \mathcal{K}, k)^{\prime} z(k+1)+(\eta v)^{2} b+2 \eta v \sum_{k=0}^{T-1} h(z, \mathcal{K}, k)^{\prime} \mathcal{Z}(k+1)\right\} \\
& =\frac{1}{1-2 b}\left(-\sigma^{2}+c+\frac{2}{\eta^{2}}\left\{\sum_{k=0}^{T-1} h(z, \mathcal{K}, k)^{\prime} z(k+1)+2 \eta v \sum_{k=0}^{T-1} h(z, \mathcal{K}, k)^{\prime} \mathcal{Z}(k+1)\right\}\right) \\
& =\frac{1}{(1-2 b)}\left(-\sigma^{2}+c+\frac{1}{\eta^{2}}\left(c_{0}+2 c_{1} \eta v\right)\right) .
\end{aligned}
$$

Substituindo (5.11) em (4.31), encontra-se:

$$
\lambda(T)=\alpha(T)+\eta v=\alpha(T)+\frac{a 2 \nu(T)+c_{1}}{(1-2 b)} .
$$

Eliminando $v$ em (5.11) e (5.13) e resolvendo para $\eta$ resulta:

$$
\eta=\sqrt{\frac{c_{0}(1-2 b)+c_{1}^{2}}{(1-2 b)\left(\sigma^{2}-c\right)+a^{2}}} .
$$

Finalmente, lembrando que $2 \nu(T)=\eta$ e substituindo $\eta$ por (5.14), revela-se a equação de $\nu(T)$ para o problema $P M V\left(\sigma^{2}\right)$.

Sob a lei de controle (4.5), a esperança e a variância da riqueza do investidor em qualquer instante de tempo foram analiticamente descritas no Teorema 4.2. Entretanto, para o $P M V\left(\sigma^{2}\right)$ é possível reescrever (4.14) e (4.15) para o instante final $t=T$, em uma formulação mais simples, conforme abaixo.

Corolário 5.3 Seja $\ell(t)=\alpha(t) \geq 0$ para $t \in \mathcal{T}, \operatorname{com} \alpha(T)>0, \nu(t)=\rho(t)=\omega(t)=$ 0 para $t \neq T, \rho(T)=\nu(T)$. Sob a lei de controle (4.5), o valor esperado e a variância 
da riqueza final são descritos analiticamente pelas equações:

$$
\begin{gathered}
E\left(V^{u}(T)\right)=\frac{1}{(1-2 b)}\left(a+\frac{c_{1}}{2 \nu(T)}\right), \\
\operatorname{Var}\left(V^{u}(T)\right)=c-\frac{a^{2}}{(1-2 b)}+\frac{c_{1}^{2}+c_{0}(1-2 b)}{4 \nu(T)^{2}(1-2 b)},
\end{gathered}
$$

com $\nu(T), a, b, c, c_{0}$ e c $c_{1}$ dados por (5.10), (5.4), (5.5) e (5.6).

Prova. As formulações analíticas para o valor esperado e variância da riqueza final são encontradas de (5.11) e (5.13), fazendo $\eta=2 \nu(T)$. Assim de (5.11) e (5.13):

$$
E\left(V^{u}(T)\right)=\frac{1}{(1-2 b)}\left(a+\frac{c_{1}}{\eta}\right)=\frac{1}{(1-2 b)}\left(a+\frac{c_{1}}{2 \nu(T)}\right)
$$

$\mathrm{e}$

$$
\begin{aligned}
\operatorname{Var}\left(V^{u}(T)\right) & =E\left(V^{u}(T)^{2}\right)-E\left(V^{u}(T)\right)^{2} \\
& =\left(c+\frac{1}{\eta^{2}}\left(c_{0}+\frac{2 c_{1} \eta}{(1-2 b)}\left(a+\frac{c_{1}}{\eta}\right)\right)\right)-\frac{1}{(1-2 b)}\left(\left(a+\frac{c_{1}}{\eta}\right)\right)^{2} \\
& =c-\frac{a^{2}}{(1-2 b)}+\frac{-2 a \eta c_{1}-c_{1}^{2}+c_{0}(1-2 b)+2 a c_{1} \eta+2 c_{1}^{2}}{\eta^{2}(1-2 b)} \\
& =c-\frac{a^{2}}{(1-2 b)}+\frac{c_{1}^{2}+c_{0}(1-2 b)}{4 \nu(T)^{2}(1-2 b)}
\end{aligned}
$$

que representam, respectivamente, as equações (5.15) e (5.16) desejadas.

A curva que agrega o conjunto de todas as combinações ótimas de média-variância no instante final é chamado de fronteira eficiente de investimentos. Esta curva revela ao investidor as relações ótimas entre risco (variância) e retorno (média) no horizonte de investimento. Para os problemas de média-variância $P M V\left(\sigma^{2}\right)$ e $P M V(\epsilon)$ com restrição apenas no instante final a fronteira eficiente de investimentos pode ser derivada analiticamente conforme abaixo. 
Corolário 5.4 A fronteira eficiente de investimentos dos problemas de média variância $\operatorname{PMV}\left(\sigma^{2}\right)$ e $\operatorname{PMV}(\epsilon)$ com restrição apenas no instante final é definida pela equação:

$$
\operatorname{Var}\left(V^{u}(T)\right)=c-\frac{a^{2}}{(1-2 b)}+\frac{\left(c_{1}^{2}+c_{0}(1-2 b)\right)(1-2 b)}{c_{1}^{2}}\left(E\left(V^{u}(T)\right)-\frac{a}{(1-2 b)}\right)^{2}
$$

onde $\operatorname{Var}(V(T))$ e $E(V(T))$ são a variância e o valor esperado da riqueza final, e a, $b, c, c_{0}$ e c c são como em (5.4), (5.5) e (5.6).

Prova. Combinando (5.15) e (5.16) e eliminando o parâmetro $v(T)$, encontra-se:

$$
\begin{aligned}
\operatorname{Var}\left(V^{u}(T)\right) & =c-\frac{a^{2}}{(1-2 b)}+\frac{c_{1}^{2}+c_{0}(1-2 b)}{4\left(\frac{c_{1}}{2\left(E\left(V^{u}(T)\right)(1-2 b)-a\right)}\right)^{2}(1-2 b)} \\
& =c-\frac{a^{2}}{(1-2 b)}+\frac{\left(c_{1}^{2}+c_{0}(1-2 b)\right)\left(2\left(E\left(V^{u}(T)\right)(1-2 b)-a\right)\right)^{2}}{4 c_{1}^{2}(1-2 b)} \\
& =c-\frac{a^{2}}{(1-2 b)}+\frac{\left(c_{1}^{2}+c_{0}(1-2 b)\right)(1-2 b)}{c_{1}^{2}}\left(E\left(V^{u}(T)\right)-\frac{a}{(1-2 b)}\right)^{2}
\end{aligned}
$$

que é a expressão desejada para a fronteira eficiente como em (5.17).

O formato da equação da fronteira eficiente revela importantes características sobre o universo de escolhas ótimas disponíveis ao investidor. Esta equação tem o formato de uma curva hiperbólica com centro em $\left(0, \frac{a}{(1-2 b)}\right)$. Desta forma, a variância mínima da riqueza final $\left(\operatorname{Var}(V(T))_{\min }\right)$ é dada por:

$$
\operatorname{Var}(V(T))_{\min }=c-\frac{a^{2}}{(1-2 b)}
$$

e o valor esperado do patrimônio terminal $\left(E(V(T))_{\min }\right)$ associado a este portfólio de mínima variância é descrito pela expressão:

$$
E(V(T))_{\min }=\frac{a}{(1-2 b)}
$$




\subsection{Solução para os Problemas Multi-Período}

\section{Tradicionais de Média-Variância com}

\section{Parâmetros Sujeitos a Saltos Markovianos}

Os problemas multi-período de média-variância tradicionais com saltos Markovianos são caracterizados por terem tanto suas funções objetivo, como suas restrições incidindo apenas no instante final. Na Seção 3.3, estes problemas foram denotados como $P\left(\sigma^{2}\right)$ e $P(\epsilon)$. As soluções destes problemas para o caso em que o mercado financeiro é formado apenas por ativos de risco são derivadas nesta seção.

A diferença entre os problemas $P M V\left(\sigma^{2}\right)$ e $P M V(\epsilon)$ com restrição apenas no instante final, apresentados na seção anterior, e $P\left(\sigma^{2}\right)$ e $P(\epsilon)$ é que nos dois primeiros a função objetivo é uma soma ponderada ao longo do tempo da variância ou da esperança da riqueza, enquanto em $P\left(\sigma^{2}\right)$ e $P(\epsilon)$ a função objetivo só considera estas variáveis no horizonte de investimento. Consequentemente, a partir dos resultados encontrados na seção anterior, é possível solucionar $P\left(\sigma^{2}\right)$ e $P(\epsilon)$ já que eles representam apenas casos particulares dos problemas $P M V\left(\sigma^{2}\right)$ e $P M V(\epsilon)$ com restrição apenas no instante final. De fato, considerando para $\operatorname{LP} M V\left(\sigma^{2}\right)$ e $L P M V(\epsilon)$ que

$$
\begin{aligned}
\omega(t) & =0, \alpha(t)=0, \text { para } t \neq T, \\
\alpha(T) & =1, \omega(T)>0,
\end{aligned}
$$

em (5.2) e (5.3), resulta-se nos problemas $P\left(\sigma^{2}\right)$ e $P(\epsilon)$, depois de inserido os Multiplicadores de Lagrange $\omega(t)$, para $t \in \mathcal{T}$.

Portanto, a solução para os problemas $P\left(\sigma^{2}\right)$ e $P(\epsilon)$ é descrita nos Teoremas 5.2 e 5.1. No entanto, para $P\left(\sigma^{2}\right)$ e $P(\epsilon)$ é possível simplificar as formulações apresentadas na Seção 5.2 .

Representa-se, respectivamente, por $\Pi\left(P\left(\sigma^{2}\right)\right)$ e $\Pi(P(\epsilon))$ o conjunto de soluções ótimas dos problemas $P\left(\sigma^{2}\right)$ e $P(\epsilon)$. A solução para $P\left(\sigma^{2}\right)$ e $P(\epsilon)$ com parâmetros 
sujeitos a saltos Markovianos foi apresentada em (Çakmak e Özekici (2006)) para o caso em que o mercado é formado por um ativo de risco que também está sujeito à cadeia de Markov. O teorema a seguir apresenta a solução para $P\left(\sigma^{2}\right)$ e $P(\epsilon)$ com parâmetros sujeitos a saltos Markovianos, quando o mercado é formado apenas por ativos de risco.

Teorema 5.3 Uma estratégia de investimento ótima $u=(u(0), \ldots, u(T-1))$ para os problemas $P\left(\sigma^{2}\right)$ e $P(\epsilon)$ é dada pela lei de controle (4.5), com

$$
\lambda(T)=\frac{\ell(T)+2 \nu(T) a}{(1-2 b)}
$$

em (4.2) e

$$
\left\{\begin{array}{l}
\ell(T)=1 \text { e } \nu(T)=\sqrt{\frac{b}{2 a^{2}+2(1-2 b)\left(\sigma^{2}-c\right)}}, \text { para o problema } P\left(\sigma^{2}\right), \\
\ell(T)=\frac{\epsilon(1-2 b)-a}{b} \text { e } \nu(T)=1, \text { para o problema } P(\epsilon) .
\end{array}\right.
$$

Sob esta politica de investimento, a esperança e a variância da riqueza final e a fronteira eficiente de investimentos são, respectivamente, obtidas pelas expressões:

$$
\begin{gathered}
E\left(V^{u}(T)\right)=\frac{a \nu(T)+\ell(T) b}{\nu(T)(1-2 b)}, \\
\operatorname{Var}\left(V^{u}(T)\right)=c-\frac{a^{2}}{(1-2 b)}+\frac{\ell(T)^{2} b}{2 \nu(T)^{2}(1-2 b)},
\end{gathered}
$$

$e$

$$
\operatorname{Var}\left(V^{u}(T)\right)=\left(c-\frac{a^{2}}{(1-2 b)}\right)+\frac{(1-2 b)}{2 b}\left(E(V(T))-\frac{a}{(1-2 b)}\right)^{2}
$$

com $a, b$ e c dados por (5.4).

Prova. A prova da lei de controle segue dos Teoremas 5.1 e 5.2. Para encontrar (5.18), (5.19), (5.20) e (5.21), recorda-se que $\nu(t)=\rho(t)=\ell(t)=0$ para $t \neq T$, e segue-se 
procedimento similar ao utilizado nos Corolários 5.2 e 5.3. A prova para a fronteira eficiente é similar à de (5.17).

Observação 5.1 De fato, os resultados apresentados nesta seção representam uma simplificação dos problemas $P M V\left(\sigma^{2}\right)$ e PMV $(\epsilon)$. No entanto, a apresentação mais detalhada destes se mostra interessante para demonstrar que os resultados apresentados neste trabalho generalizam aqueles expostos em Çakmak e Özekici (2006), pois as soluções descritas no Teorema 5.3 são casos particulares dos resultado anteriores e coincidem com as exibidas em Çakmak e Özekici (2006). Esta coincidência ocorre apesar do mercado financeiro aqui utilizado ser formado apenas por ativos de risco. Portanto, verifica-se que o fato de Çakmak e Özekici (2006) ter utilizado um ativo livre de risco sujeito à cadeia de Markov, não produz simplificação ao modelo.

\subsection{Modelo no qual o Mercado é Formado por $n$ Ativos de Risco e um Ativo Livre de Risco}

Embora o caso no qual existe um ativo livre de risco possa ser considerado como um caso particular daquele em que o mercado é formado apenas por ativos de risco, ele possui alguns resultados interessantes que validam sua investigação para o caso dos problemas $P\left(\sigma^{2}\right)$ e $P(\epsilon)$.

\subsubsection{Simplificações no Mercado}

Entende-se por ativo livre de risco aquele que não possui nenhuma fonte de incerteza, ou seja, não possui volatilidade e também não é afetado pelos diferentes modos do mercado, ou seja não é modulado por uma cadeia de Markov. Apesar da suposição de existência de um ativo livre de risco não ser real, ou seja, todos os ativos possuem alguma fonte de incerteza, este caso pode ser visto como uma conveniente simplificação, já que, 
usualmente, a taxa básica de juros se altera de forma pouco frequente, sendo modificada através de decisões tomadas pelos bancos centrais em períodos predeterminados.

Assume-se o ativo $i=0$ como aquele livre de risco. Como foi definido, o ativo livre de risco não possui volatilidade e não é modulado pelos modos do mercado, logo $\sigma_{0}(t, i)=0$ e $\mu_{0}(t, i)=r_{f}(t)$ para todo $t=0, \ldots, T$ e $i \in \mathcal{M}$. Portanto, o processo de retorno dos preços $\overline{\mathcal{R}}(t)=\left(\mathcal{R}_{0}(t), \ldots, \mathcal{R}_{n}(t)\right)^{\prime}$ evolui conforme (3.5), considerando que:

$$
\mathcal{R}_{0}(t+1)=1+r_{f}(t)
$$

O fato da volatilidade ser nula e da rentabilidade ser determinística para o ativo livre de risco, permite que se realizem uma série de simplificações nas equações do modelo proposto. Portanto, para cada $i \in \mathcal{M}$ e $t=0, \ldots, T$ reescreve-se (3.10), (3.19), (3.20), (3.25) e (3.23), respectivamente, como:

$$
\begin{gathered}
A_{i}(t)=\hat{A}(t)=1+r_{f}(t), \\
\delta_{i}(t)=E\left(A_{i}(t)^{2}\right)=\hat{A}(t)^{2}, \\
\varphi_{i}(t)^{\prime}=E\left(A_{i}(t) B_{i}(t)^{\prime}\right)=\hat{A}(t) E\left(B_{i}(t)^{\prime}\right)=\hat{A}(t) \chi_{i}(t)^{\prime}, \\
R_{i}(t)=\hat{A}(t)\left(1-\beta_{i}(t)\right)
\end{gathered}
$$

e

$$
Q_{i}(t)=\delta_{i}(t)\left(1-\beta_{i}(t)\right)=\hat{A}(t)^{2}\left(1-\beta_{i}(t)\right)=\hat{A}(t) R_{i}(t) .
$$

Adicionalmente define-se:

$$
\vartheta(t)=\prod_{k=t}^{T-1} \hat{A}(k)
$$


e

$$
\mathcal{H}(t)=\left(\prod_{k=t}^{T-1}(I-\beta(k)) P(k)\right) e
$$

permitindo a realização das seguintes simplificações nas equações em (4.3), (4.4), (4.20) e (5.4) para:

$$
\begin{gathered}
\mathcal{Z}(t)=\vartheta(t) \mathcal{H}(t), \\
\mathcal{K}(t)=\vartheta(t) \mathcal{Z}(t), \\
h(Z, K, t)^{\prime}=\frac{1}{2 \vartheta(t+1)} \pi(t)^{\prime} \beta(t) P(t), \\
a=\vartheta(0) V(0) \pi(0)^{\prime} \mathcal{H}(0), c=(\vartheta(0) V(0))^{2} \pi(0)^{\prime} \mathcal{H}(0)
\end{gathered}
$$

e

$$
b=\frac{1}{2}\left(1-\pi(0)^{\prime} \mathcal{H}(0)\right)
$$

As simplificações realizadas acima são facilmente obtidas apenas por substituição ou por meras manipulações algébricas. Com exceção da derivação de (5.33), a qual é descrita a seguir. Para demonstrar a equação (5.33), ou seja, que:

$$
\sum_{k=0}^{T-1} \pi(t)^{\prime} \beta(t) P(t) \mathcal{H}(t+1)=1-\pi(0)^{\prime} \mathcal{H}(0)
$$

inicialmente, soma-se e subtrai-se o termo $\pi(0)^{\prime} \mathcal{H}(0)$ ao lado esquerdo da equação 
acima e expande-se o somatório, assim:

$$
\begin{aligned}
\sum_{k=0}^{T-1} \pi(t)^{\prime} \beta(t) P(t) \mathcal{H}(t+1) & =\pi(0)^{\prime} \mathcal{H}(0)+\pi(0)^{\prime} \beta(0) P(0) \mathcal{H}(1) \\
& +\pi(1)^{\prime} \beta(1) P(1) \mathcal{H}(2)+\ldots \\
+\pi(T-2)^{\prime} \beta & (T-2) P(T-2) \mathcal{H}(T-1) \\
& +\pi(T-1)^{\prime} \beta(T-1) P(T-1) e-\pi(0)^{\prime} \mathcal{H}(0) .
\end{aligned}
$$

Lembrando que

$$
\mathcal{H}(l)=[(I-\beta(l)) P(l)] \mathcal{H}(l+1)
$$

e colocando em evidência o termo $\pi(0)^{\prime}$ na equação acima, chega-se a:

$$
\begin{aligned}
\sum_{k=0}^{T-1} \pi(t)^{\prime} \beta(t) P(t) \mathcal{H}(t+1)= & \pi(0)^{\prime}[(I-\beta(0)) P(0)+\beta(0) P(0)] \mathcal{H}(1) \\
& +\pi(1)^{\prime} \beta(1) P(1) \mathcal{H}(2) \\
& +\pi(2)^{\prime} \beta(2) P(2) \mathcal{H}(3)+\ldots \\
& +\pi(T-2)^{\prime} \beta(T-2) P(T-2) \mathcal{H}(T-1) \\
& +\pi(T-1)^{\prime} \beta(T-1) P(T-1) e-\pi(0)^{\prime} \mathcal{H}(0),
\end{aligned}
$$

ou seja,

$$
\begin{aligned}
\sum_{k=0}^{T-1} \pi(t)^{\prime} \beta(t) P(t) \mathcal{H}(t+1)= & \pi(0)^{\prime} P(0) \mathcal{H}(1) \\
& +\pi(1)^{\prime} \beta(1) P(1) \mathcal{H}(2) \\
& +\pi(2)^{\prime} \beta(2) P(2) \mathcal{H}(3)+\ldots \\
& +\pi(T-2)^{\prime} \beta(T-2) P(T-2) \mathcal{H}(T-1) \\
& +\pi(T-1)^{\prime} \beta(T-1) P(T-1) e-\pi(0)^{\prime} \mathcal{H}(0) .
\end{aligned}
$$

Recordando que $\pi(t)^{\prime} P(t)=\pi(t+1)^{\prime}$ e refazendo o mesmo procedimento acima, 
encontra-se:

$$
\begin{aligned}
\sum_{k=0}^{T-1} \pi(t)^{\prime} \beta(t) P(t) \mathcal{H}(t+1)= & \pi(1)^{\prime}[(I-\beta(1)) P(1)+\beta(1) P(1)] \mathcal{H}(2) \\
& +\pi(2)^{\prime} \beta(2) P(2) \mathcal{H}(3)+\ldots \\
& +\pi(T-2)^{\prime} \beta(T-2) P(T-2) \mathcal{H}(T-1) \\
& +\pi(T-1)^{\prime} \beta(T-1) P(T-1) e-\pi(0)^{\prime} \mathcal{H}(0) .
\end{aligned}
$$

Repetindo este procedimento até chegarmos ao fim do somatório, obtém-se:

$$
\begin{aligned}
& \sum_{k=0}^{T-1} \pi(t)^{\prime} \beta(t) P(t) \mathcal{H}(t+1) \\
= & \pi(2)^{\prime} \mathcal{H}(2)+\pi(2)^{\prime} \beta(2) P(2) \mathcal{H}(3)+\ldots+\pi(T-2)^{\prime} \beta(T-2) P(T-2) \\
& \cdot \mathcal{H}(t-1)+\pi(T-1)^{\prime} \beta(T-1) P(T-1) e-\pi(0)^{\prime} \mathcal{H}(0) \\
= & \pi(T-1)^{\prime} \mathcal{H}(t-1)+\pi(T-1)^{\prime} \beta(T-1) P(T-1) e-\pi(0)^{\prime} \mathcal{H}(0) \\
= & \pi(T-1)^{\prime}(I-\beta(T-1)) P(T-1) e+\pi(T-1)^{\prime} \beta(T-1) P(T-1) e \\
& -\pi(0)^{\prime} \mathcal{H}(0) \\
= & \pi(T-1)^{\prime} P(T-1) e-\pi(0)^{\prime} \mathcal{H}(0) \\
= & \pi(T)^{\prime} e-\pi(0)^{\prime} \mathcal{H}(0) .
\end{aligned}
$$

Como, por definição, $\pi(l)^{\prime} e=1$, determina-se que:

$$
\sum_{k=0}^{T-1} \pi(t)^{\prime} \beta(t) P(t) \mathcal{H}(t+1)=1-\pi(0)^{\prime} \mathcal{H}(0)
$$

finalizando a demonstração da equação (5.33). 


\subsubsection{Solução para os Problemas no Caso em que Existe um Ativo Livre de Risco}

O procedimento para encontrar a lei de controle ótima para o caso em que um dos ativos é livre de risco é similar ao caso em que todos os ativos são de risco. Dessa forma, expressa-se abaixo apenas os resultados que se diferenciam do caso geral estudado quando todos os ativos são de risco.

Teorema 5.4 Uma política ótima de investimentos $u=(u(0), \ldots, u(T-1))$ para os problemas $P\left(\sigma^{2}\right)$ e $P(\epsilon)$, considerando que o ativo $i=0$ é livre de risco, é dada pela lei de controle:

$$
u(t)=-\phi_{\theta(t)}(t)^{-1} \chi_{\theta(t)}(t)\left(\hat{A}(t) V(t)+\frac{\lambda(T)}{2 \rho(T) \vartheta(t+1)}\right)
$$

com $\lambda(T)$ descrito como em (5.18),

$$
\left.\begin{array}{l}
E\left(V^{u}(T)\right)=\epsilon, \nu(T)=\rho(T)=1, \\
\ell(T)=\frac{2 \pi(0)^{\prime} \mathcal{H}(0)(\epsilon-\vartheta(0) V(0))}{1-\pi(0)^{\prime} \mathcal{H}(0)},
\end{array}\right\} \text { para o problema } P(\epsilon),
$$

$\nu(t)=\rho(t)=\ell(t)=0$ para $t \neq T$ e com $a, b$ e c dados por (5.32) e (5.33). Além disso, para esta lei de controle ótima, a esperança e a variância da riqueza final e a fronteira eficiente de investimentos são descritos, respectivamente, por:

$$
\begin{gathered}
E(V(T))=\vartheta(0) V(0)+\frac{\ell(T)\left(1-\pi(0)^{\prime} \mathcal{H}(0)\right)}{2 \nu(T) \pi(0)^{\prime} \mathcal{H}(0)} \\
\operatorname{Var}(V(T))=\frac{\ell(T)^{2}\left(1-\pi(0)^{\prime} \mathcal{H}(0)\right)}{2 \nu(T)^{2} \pi(0)^{\prime} \mathcal{H}(0)}
\end{gathered}
$$


$e$

$$
\operatorname{Var}(V(T))=\frac{1-\pi(0)^{\prime} \mathcal{H}(0)}{\pi(0)^{\prime} \mathcal{H}(0)}(E(V(T))-\vartheta(0) V(0))^{2}
$$

Prova. A prova deste teorema segue diretamente do Teorema 5.3, observando que a e $c$ são como em (5.32), e que em (4.5), utilizando-se de (5.29) e (5.30), tem-se que $\frac{\mathcal{E}_{i}(Z(t+1))}{\mathcal{E}_{i}(K(t+1))}=\frac{\lambda(T)}{2 \rho(T) \vartheta(t+1)}$.

Como consequência da existência de um ativo livre de risco, a variância mínima do patrimônio terminal $\left(\operatorname{Var}(V(T))_{\min }\right)$ na fronteira eficiente é zero e o valor esperado da riqueza neste instante de tempo é determinado por:

$$
E(V(T))_{\min }=\vartheta(0) V(0)
$$

Observação 5.2 Ressalta-se que os resultados obtidos tanto nos Teoremas 5.3 e 5.4 coincidem com aqueles apresentados em Li e $\mathrm{Ng}$ (2000) para o caso em que os parâmetros não são modulados por uma cadeia de Markov. 


\section{Capítulo 6}

\section{Seleção de Carteiras para Superar um}

\section{Benchmark}

Nesta seção, estende-se a teoria desenvolvida nos capítulos anteriores na investigação de um problema que é enfrentado pela maioria dos administradores de recursos profissionais. Trata-se do problema de se otimizar um portfólio visando a superação de um benchmark. Os gestores de recursos, em sua maioria, têm como meta de rentabilidade superar um determinado benchmark. Para tanto, têm como controle de risco uma medida relacionada a este objetivo, ou seja, a variância do tracking error. Esta forma de atuação é utilizada como uma ferramenta de premiação, punição e apreçamento do serviço de administração de carteiras. Isto ocorre, porque os investidores possuem duas alternativas de investimento: uma carteira de perfil ativo a um benchmark, ou uma carteira passiva a este benchmark, ou seja, que replica um índice de mercado. Geralmente, esta segunda opção tem um custo (taxa de administração) mais barato para o investidor. Logo, o apreçamento do serviço de administração de carteira, assim como a premiação e a punição, devem ser baseadas no valor adicional que o gestor do portfólio consegue agregar acima do benchmark escolhido, e do risco associado para atingir esta 
meta. Como consequência, estes fatores determinam a relevância da otimização de um portfólio com o objetivo de superar um índice de mercado.

Inicialmente apresentam-se as características que envolvem o modelo do tracking error. Em seguida demonstra-se o modelo principal a ser investigado: o problema multiperíodo generalizado de média-variância do tracking error (TE) com saltos Markovianos (PGMVTE). Passa-se então à uma breve descrição dos modelos que derivam do PGMVTE. Finalmente apresentam-se as soluções dos modelos.

\subsection{O Tracking Error do Portfólio}

Como descrito na Seção 3.2, seja $\bar{u}(t)=\left(u_{0}(t), u(t)\right)^{\prime} \in \mathbb{R}^{n+1}, \operatorname{com} u(t)=\left(u_{1}(t), \ldots\right.$, $\left.u_{n}(t)\right)^{\prime}$, definido como o vetor que representa o montante da riqueza alocado nos $n+1$ ativos.

Considere um portfólio benchmark o qual o investidor deseja superar. Define-se $\bar{u}_{B}(t)=\left(u_{B_{0}}(t), u_{B}(t)\right)^{\prime} \in \mathbb{R}^{n+1}, \operatorname{com} u_{B}(t)=\left(u_{B_{1}}(t), \ldots, u_{B_{n}}(t)\right)^{\prime}$, como o vetor que representa o montante investido em cada ativo do benchmark, replicando a participação deste no benchmark. Vale ressaltar que a carteira futura do benchmark não é conhecida ex-ante, pois cada ativo que a compõe segue um processo como definido em (3.5), tornando a dinâmica dos pesos dos ativos estocástica.

Assume-se que o universo de ativos disponíveis para investimento pelo aplicador contenha todos os ativos do benchmark escolhido e que o portfólio benchmark seja constituído pelo mesmo número de ativos do universo de investimento do investidor, ou seja, $n+1$ ativos. Logo, se o ativo $j$ faz parte do universo de investimento, mas de fato não pertence ao benchmark escolhido, ele terá participação nula na carteira do benchmark aqui considerada.

Chama-se de $\varkappa_{j}(t)$ a diferença entre o montante aplicado no ativo $j$ da carteira do investidor e o valor do ativo $j$ correspondente na carteira benchmark no instante $t$. $\mathrm{O}$ 
vetor com estas diferenças é definido como $\bar{\varkappa}(t)=\left(\varkappa_{0}(t), \varkappa(t)\right)^{\prime} \in \mathbb{R}$, com

$$
\varkappa_{0}(t)=\left(u_{0}(t)-u_{B_{0}}(t)\right)
$$

e

$$
\varkappa(t)=\left(\varkappa_{1}(t), \ldots, \varkappa_{n}(t)\right)=\left(\left(u_{1}(t)-u_{B_{1}}(t)\right), \ldots,\left(u_{n}(t)-u_{B_{n}}(t)\right)\right) .
$$

Observa-se que $\varkappa_{j}(t)$ pode assumir valores positivos ou negativos, dependendo se o valor do ativo alocado no portfólio do investidor é maior ou menor que aquele no benchmark.

Definição 6.1 O conjunto de estratégias admissíveis de alocação em excesso a um benchmark $\mathcal{X}=\{\varkappa=(\bar{\varkappa}(0), \ldots, \bar{\varkappa}(T-1))\}$ é tal que para cada $t=0, \ldots, T-1$, $\bar{\varkappa}(t)=\left(\varkappa_{0}(t), \ldots, \varkappa_{n}(t)\right)^{\prime}$ é um vetor aleatório $\mathcal{F}_{t}$-mensurável, tomando valores em $\mathbb{R}^{n+1}$.

Associado a cada estratégia de alocação em excesso a um benchmark $\varkappa$, há uma estratégia $u$, como definida em 3.1, que determina o processo do valor do portfólio do investidor como $\left\{V^{u}(t) \in \mathbb{R} ; t=0, \ldots, T\right\}$, representando a riqueza deste ao final do instante $t$. Similarmente, o processo do valor do portfólio benchmark é descrito por $\left\{V^{u_{B}}(t) \in \mathbb{R} ; t=0, \ldots, T\right\}, \operatorname{com} V^{u_{B}}(0)=V^{u}(0)$.

Representa-se o ganho (perda) do investidor acima (abaixo) do benchmark até o instante $t$ como a diferença entre a riqueza do investidor e o valor do portfólio benchmark, ou seja,

$$
G(t)=V^{u}(t)-V^{u_{B}}(t)
$$

com

$$
G(0)=0
$$

A variável $G(t)$ foi definida por Roll (1992) como o Tracking Error (TE) do portfólio 
do investidor. Assim, o TE do portfólio do investidor no instante $t$ é expresso por:

$$
G(t)=\bar{\varkappa}(t)^{\prime} \bar{e}=\varkappa_{0}(t)+\varkappa(t)^{\prime} e .
$$

Nota-se que $x_{0}(t)$ é determinado por $G(t)-\left(\sum_{j=1}^{n} \varkappa_{j}(t)\right)$, portanto, pode ser eliminado do processo de otimização.

Assumindo que a carteira do investidor e do índice benchmark sejam auto-financiadas, e a riqueza inicial do aplicador seja $V(0)=V_{0}>0$, o retorno adicional ao benchmark do investidor evolui conforme:

$$
G(t+1)=\bar{\varkappa}(t)^{\prime} \overline{\mathrm{R}}_{\theta(t)}(t)
$$

Recorda-se de $(3.5)$ que $\overline{\mathrm{R}}_{\theta(t)}(t)=(\bar{e}+\bar{\mu}(t, \theta(t))+\bar{\sigma}(t, \theta(t)) W(t))$. Portanto:

$$
\begin{aligned}
G(t+1)= & \bar{\varkappa}(t)^{\prime}(\bar{e}+\bar{\mu}(t, \theta(t))+\bar{\sigma}(t, \theta(t)) W(t)) \\
= & \bar{\varkappa}(t)^{\prime} \bar{e}+\bar{\varkappa}(t)^{\prime}(\bar{\mu}(t, \theta(t))+\bar{\sigma}(t, \theta(t)) W(t)) \\
= & G(t)+\varkappa_{0}(t)\left(\bar{\mu}_{0}(t, \theta(t))+\bar{\sigma}_{0}(t, \theta(t)) W(t)\right) \\
& +\varkappa(t)^{\prime}(\mu(t, \theta(t))+\sigma(t, \theta(t)) W(t)) .
\end{aligned}
$$

Substituindo $\varkappa_{0}(t)$ de (6.1), obtém-se:

$$
\begin{aligned}
G(t+1)= & G(t)+\left(G(t)-\varkappa^{\prime}(t) e\right)\left(\bar{\mu}_{0}(t, \theta(t))+\bar{\sigma}_{0}(t, \theta(t)) W(t)\right) \\
& +\varkappa(t)^{\prime}(\mu(t, \theta(t))+\sigma(t, \theta(t)) W(t)) \\
= & \left(1+\bar{\mu}_{0}(t, \theta(t))+\bar{\sigma}_{0}(t, \theta(t)) W(t)\right) G(t) \\
& +\varkappa(t)^{\prime}\left(\mu(t, \theta(t))-e \mu_{0}(t, \theta(t))\right. \\
& \left.+\left(\sigma(t, \theta(t))-e \sigma_{0}(t, \theta(t))\right) W(t)\right),
\end{aligned}
$$

ou seja,

$$
G(t+1)=A_{\theta(t)}(t) G(t)+B_{\theta(t)}(t)^{\prime} \varkappa(t),
$$


onde $A_{\theta(t)}(t)$ e $B_{\theta(t)}(t)$ são definidos como em (3.15) e (3.16), respectivamente.

A estrutura de (6.2) é similar a de (3.14) introduzida na Seção 3.1. Portanto, considerando a formulação (6.2), as equações (3.17) a (3.26) também podem ser aplicadas neste capítulo.

\subsection{Formulação do Problema Generalizado de}

\section{Média-Variância do Tracking Error com Saltos}

\section{Markovianos}

O problema generalizado de média-variância (3.32) pode ser reformulado para o problema generalizado de média-variância do tracking error (TE) com saltos Markovianos, ou PGMVTE. Para isso, basta substituir a esperança da riqueza $E(V(t))$ pela esperança do TE do portfólio $E(G(t))$, e trocar o valor esperado do quadrado da riqueza $E\left(V(t)^{2}\right)$ pela esperança do quadrado do TE da carteira $E\left(G(t)^{2}\right)$.

Portanto, o problema PGMVTE também é um problema de controle estocástico sem restrição, mas que objetiva maximizar a soma ponderada ao longo do tempo dos seguintes elementos: o valor esperado do quadrado do tracking error do portfólio, a esperança do TE da carteira e o quadrado deste último elemento. Como definido na Seção 3.3, considere uma sequência de números positivos $\rho(t), \nu(t)$, e uma sequência de números reais $\ell(t)$, com $\rho(T)>0, \nu(T)>0$ e $\ell(T) \neq 0$, para $t \in \mathcal{I}:=\left\{\tau_{1}, \ldots, \tau_{\iota_{f}}\right\}$, e para $t \notin \mathcal{I}$ e para o momento inicial $\tau_{0}=0$ com $\ell(t)=\nu(t)=\rho(t)=0$. Define-se, portanto o problema o PGMVTE como:

$$
P G M V T E(\rho, \ell, \nu): \max _{\varkappa \in \mathcal{X}} \sum_{t \in \mathcal{I}}\left(\nu(t) E(G(t))^{2}-\rho(t) E\left(G(t)^{2}\right)+\ell(t) E(G(t))\right) .
$$

Como descrito na Subseção 3.3.2, tendo em vista a característica de ser não separável no sentido da programação dinâmica, associa-se a $\operatorname{PGMVTE}(\rho, \ell, \nu)$ uma formulação auxiliar como a desenvolvida em Li e Ng (2000). Assim, seja $\lambda(t)$ um con- 
junto de números reais, para $t=1, \ldots, T$, com $\lambda(t)=0$ para $t \notin \mathcal{I}$, atribui-se a PGMVTE $(\rho, \ell, \nu)$ o seguinte problema auxiliar:

$$
\operatorname{ATE}(\lambda, \rho): \min _{\varkappa \in \mathcal{X}} \sum_{t=1}^{T} E\left\{\rho(t) G(t)^{2}-\lambda(t) G(t)\right\} .
$$

\subsubsection{Formulações Derivadas do PGMVTE}

Os problemas de média-variância (3.27) a (3.31) também podem ser reescritos com o enfoque do tracking error, fazendo as devidas substituições, ou seja, substituindo a esperança da riqueza $E(V(t))$ pela esperança do TE do portfólio $E(G(t))$, e a variância da carteira $\operatorname{Var}(V(t))$ pela variância do tracking error do portfólio $\operatorname{Var}(G(T))$. A seguir se descreve os principais problemas.

Os problemas tradicionais de média-variância em multi-período do tracking error buscam selecionar um conjunto de portfólios que proporcionem o maior TE final esperado para um nível de risco selecionado para o instante final (variância do TE final), ou encontrar uma sequência de portfólios que produzam a menor variância do TE terminal do portfólio para um desejado valor esperado para este TE neste mesmo período.

Formalmente, as duas formulações citadas acima, nomeadas respectivamente de PTE $\left(\sigma^{2}\right)$ e PTE $(\epsilon)$, podem ser expressas como:

$$
\begin{aligned}
\operatorname{PTE}\left(\sigma^{2}\right) & : \max _{\varkappa \in \mathcal{X}} E(G(T)) \\
\text { sujeito a } & : \operatorname{Var}(G(T)) \leq \sigma^{2}
\end{aligned}
$$

e

$$
\begin{array}{ll}
\operatorname{PTE}(\epsilon) & : \max _{\varkappa \in \mathcal{X}}-\operatorname{Var}(G(T)) \\
\text { sujeito a } & : \quad E(G(T)) \geq \epsilon .
\end{array}
$$


Considerando que a função objetivo é maximizar a soma ponderada do valor esperado do TE do tempo, tendo como restrição o risco (variância do TE) em cada instante $t \in \mathcal{T}$, ou que a função objetivo é minimizar a soma ponderada da variância do TE ao longo do tempo, tendo como restrição a esperança do TE em cada instante $t \in \mathcal{T}$, reformulam-se os problemas $P M V\left(\sigma^{2}\right)$ e $P M V(\epsilon)$, denominando-os, respectivamente de PMVTE $\left(\sigma^{2}\right)$ e PMVTE $(\epsilon)$, como:

$$
\begin{aligned}
& \operatorname{PMVTE}\left(\sigma^{2}\right): \max _{\varkappa \in \mathcal{X}} \sum_{t \in \mathcal{T}} \alpha(t) E(G(t)) \\
& \text { sujeito a : } \operatorname{Var}(G(t)) \leq \sigma^{2}(t), t \in \mathcal{T}
\end{aligned}
$$

e

$$
\begin{aligned}
P M \operatorname{PTE}(\epsilon) & : \max _{\varkappa \in \mathcal{X}}-\sum_{t \in \mathcal{T}} \alpha(t) \operatorname{Var}(G(t)) \\
\text { sujeito a } & : \quad E(G(t)) \geq \epsilon(t), t \in \mathcal{T} .
\end{aligned}
$$

Portanto, a relevância que o investidor atribui a cada instante de tempo $t \in \mathcal{T}$ pode ser controlada através do parâmetro $\alpha(t)$ como definido na Seção 3.3. Quanto maior for o valor atribuído a este coeficiente em um determinado instante em relação aos outros valores de $\alpha(t)$, maior será a importância da esperança ou da variância do TE neste período em relação aos outros instantes de tempo no processo de otimização.

Outro problema que os investidores enfrentam com o enfoque do tracking error é aquele que deriva do problema $P B C(\varrho, \psi)$, descrito na Seção 3.3. Neste caso, a função objetivo seria a maximização da soma ponderada do valor esperado do TE do portfólio ao longo do tempo, tendo como restrição a probabilidade máxima $(\varrho(t))$ do TE da carteira cair abaixo de um determinado valor $(\psi(t))$ selecionado pelo investidor. Este 
problema é formulado como:

$$
\begin{aligned}
P B C T E(\varrho, \psi) & : \max _{\varkappa \in \mathcal{X}} \sum_{t \in \mathcal{T}} \alpha(t) E(G(t)) \\
\operatorname{Var}(G(t)) & \leq \varrho(t)[E(G(t))-\psi(t)]^{2}, t \in \mathcal{T} .
\end{aligned}
$$

Os problemas (6.5) a (6.9) podem ser reescritos na formulação generalizada (6.3), seguindo os mesmos passos descritos na Subseção 3.3.1.

\subsection{Solução do Problema PGMVTE}

Tendo em vista a similaridade das formulações, os resultados encontrados nos Capítulos (4) e (5) continuam valendo para o problema de seleção de carteiras para superar um benchmark, realizando-se as devidas substituições. Repete-se então os principais resultados.

Teorema 6.1 A lei de controle ótima $\varkappa^{*}=(\varkappa(0), \ldots, \varkappa(T-1))$ para o problema (6.3), é definida como:

$$
\varkappa(t)=-\phi_{\theta(t)}(t)^{-1} \varphi_{\theta(t)}(t) G(t)-\frac{\mathcal{E}_{\theta(t)}[Z(t+1)]}{2 \mathcal{E}_{\theta(t)}[K(t+1)]} \phi_{\theta(t)}(t)^{-1} \chi_{\theta(t)}(t),
$$

com $\chi_{\theta(t)}(t), \varphi_{\theta(t)}(t), \phi_{\theta(t)}(t), Z(t)$ e $K(t)$ dados por (3.17), (3.20), (3.18) e (4.2), e com $\lambda(t)=\ell(t)+2 \nu(t) E\left(G^{\varkappa}(t)\right)$ em (4.2). Sob a lei de controle ótima $\varkappa^{*}$, o valor esperado e a variância do tracking error do portfólio em cada instante de tempo $\tau_{\kappa} \in \mathcal{I}$ são expressos, respectivamente, como:

$$
E\left(G\left(\tau_{\kappa}\right)\right)=-\frac{1}{2} \sum_{k=0}^{\kappa-1} Z\left(\tau_{k+1}\right)^{\prime} \mathcal{B}\left(\tau_{k}\right)^{\prime} e
$$

$e$

$$
\operatorname{Var}\left(G\left(\tau_{\kappa}\right)\right)=\frac{1}{4} \sum_{k=0}^{\kappa-1} \operatorname{diag}\left(Z_{i}\left(\tau_{k+1}\right)^{2}\right)^{\prime} \mathcal{C}(k)^{\prime} e-\left(\frac{1}{2} \sum_{k=0}^{\kappa-1} Z\left(\tau_{k+1}\right)^{\prime} \mathcal{B}\left(\tau_{k}\right)^{\prime} e\right)^{2}
$$


com $G(0)=0$, e $\mathcal{B}(k)$ e $\mathcal{C}(k)$ dados por (4.10) e (4.11), respectivamente.

Prova. A prova segue diretamente dos Teoremas 4.2 e 4.3 , lembrando que $G(0)=0$.

\subsection{Solução dos Problemas Derivados do PGMVTE}

As soluções dos problemas derivados do PGMVTE $(\rho, \ell, \nu)$ são similares às encontradas no Capítulo 5 e seguem o mesmo procedimento utilizado naquele capítulo. Entretanto, o fato de que $G(0)=0$, permite que se realizem algumas simplificações nas expressões derivadas naquele capítulo. Portanto, expõe-se a seguir apenas os principais resultados.

\subsubsection{Solução para os Problemas com Custo e Restrições Intermediárias}

No caso dos problemas PBCTE $(\varrho, \psi), P M V T E\left(\sigma^{2}\right)$ e PMVTE $(\epsilon)$, quando os custos e as restrições incidem nos períodos intermediários, o processo para encontrar a solução é semelhante ao descrito na Seção 5.1. Portanto, o procedimento para a solução dos problemas PBCTE $(\varrho, \psi), P M V T E\left(\sigma^{2}\right)$ e PMVTE $(\epsilon)$ é dividido em duas etapas. Na primeira etapa encontra-se a solução para o problema PGMVTE $(\rho, \ell, \nu)$. Na segunda parte, a partir da solução ótima para $P G M V T E(\rho, \ell, \nu)$, realiza-se um procedimento numérico para encontrar os Multiplicadores de Lagrange que atendem às restrições do respectivo problema PBCTE $(\varrho, \psi), \operatorname{PMVTE}\left(\sigma^{2}\right)$ ou PMVTE $(\epsilon)$. 


\subsubsection{Solução para os Problemas com Custo Intermediário e Restrições apenas no Instante Final}

Para os problemas PMVTE $\left(\sigma^{2}\right)$ e PMVTE $(\epsilon)$, quando as restrições incidem apenas no instante final, mas a função objetivo também incide sobre os períodos intermediários, é possível encontrar expressões analíticas para a solução.

Seja $\Pi\left(P M V T E\left(\sigma^{2}\right)\right)$ e $\Pi(P M V T E(\epsilon))$ o conjunto de soluções ótimas para os problemas PMVTE $\left(\sigma^{2}\right)$ e PMVTE $(\epsilon)$. Assim como descrito na Seção 5.2, a solução para estes dois problemas é apresentada nos seguintes teoremas.

Teorema 6.2 Seja $\nu(t)=\rho(t)=\alpha(t)$ para $t \in \mathcal{T}$, com $\alpha(T)>0$, e $\ell(t)=$ $\omega(t)=0$ para $t \neq T$. Se $\varkappa \in \Pi\left((P G M V T E(\rho, \ell, \nu))\right.$, com $E\left(G^{\varkappa}(T)\right)=\epsilon$, então $\varkappa \in \Pi(P M V T E(\epsilon))$.

Prova. A prova é similar à do Teorema 5.1.

Teorema 6.3 Seja $\ell(t)=\alpha(t) \geq 0$ para $t \in \mathcal{T}, \operatorname{com} \alpha(T)>0, \nu(t)=\rho(t)=\omega(t)=0$ para $t \neq T, \rho(T)=\nu(T)$. Se $\varkappa \in \Pi\left((P G M V(\rho, \ell, \nu))\right.$, com $\operatorname{Var}\left(G^{\varkappa}(T)\right)=\sigma^{2}$, então $\varkappa \in \Pi\left(P M V T E\left(\sigma^{2}\right)\right)$.

Prova. A prova é similar à do Teorema 5.2.

Sob a lei de controle (4.5), a esperança e a variância da riqueza do investidor em qualquer instante de tempo foram analiticamente descritas no Teorema 6.1. Entretanto, como comentado na Seção 5.4 .2 , para o problema PMVTE $\left(\sigma^{2}\right)$ é possível reescrever (6.11) e (6.12) em uma formulação mais simples para o instante final $t=T$, conforme abaixo.

Corolário 6.1 Seja $\ell(t)=\alpha(t) \geq 0$ para $t \in \mathcal{T}, \operatorname{com} \alpha(T)>0, \nu(t)=\rho(t)=\omega(t)=$ 0 para $t \neq T, \rho(T)=\nu(T)$. Sob a lei de controle ótima (6.10), o valor esperado e a variância da riqueza final são descritos analiticamente pelas equações:

$$
E\left(G^{u}(T)\right)=\frac{c_{1}}{2 \nu(T)(1-2 b)}
$$


$e$

$$
\operatorname{Var}\left(G^{u}(T)\right)=\frac{c_{1}^{2}+c_{0}(1-2 b)}{4 \nu(T)^{2}(1-2 b)}
$$

com $\nu(T)=\frac{1}{2} \sqrt{\frac{c_{0}(1-2 b)+c_{1}^{2}}{(1-2 b) \operatorname{Var}\left(G^{\varkappa}(T)\right)}}$, e b, $c_{0}$ e $c_{1}$ dados por (5.4), (5.5) e (5.6).

Prova. A prova segue diretamente do Corolário 5.3 .

A fronteira eficiente de investimentos para os problemas PMVTE $\left(\sigma^{2}\right)$ e PMVTE $(\epsilon)$, também pode ser simplificada em relação àquela apresentada no Corolário 5.4, conforme se observa abaixo.

Corolário 6.2 Sob a estratégia ótima de investimento (6.10) a Fronteire Eficiente de investimentos para os problemas PMVTE $\left(\sigma^{2}\right)$ e PMVTE $(\epsilon)$ com restrição apenas no instante final é descrita pela expressão:

$$
\operatorname{Var}\left(G^{u}(T)\right)=\frac{\left(c_{1}^{2}+c_{0}(1-2 b)\right)(1-2 b)}{c_{1}^{2}} E\left(G^{u}(T)\right)^{2},
$$

com $b, c_{0}$ e $c_{1}$ dados por (5.4), (5.5) e (5.6).

Prova. A prova segue diretamente do Corolário 5.4 .

Nota-se pela equação da fronteira eficiente acima que como $G(0)=0$, o ponto de mínima variância do TE, assim como o seu correspondente valor esperado do TE na fronteira eficiente são ambos zero. Percebe-se que (6.13) se diferencia de (5.17), pois o desvio padrão do TE é diretamente linear à esperança do TE. De fato, a expressão (6.13) se assemelha mais à expressão (5.37) para o caso em que existe um ativo livre de risco. A explicação para isto é que no enfoque do TE o gestor tem a possibilidade de neutralizar o risco, replicando o benchmark, que seria uma atitude semelhante a de aplicar somente no ativo livre de risco quando se utiliza o enfoque do retorno absoluto, visto anteriormente. 


\subsubsection{Solução para os Problemas com Custo e Restrições apenas no Instante Final}

O teorema a seguir apresenta a solução para os problemas PTE $\left(\sigma^{2}\right)$ e PTE $(\epsilon)$ para o caso em que todos os ativos do universo de investimento são de risco.

Teorema 6.4 Uma estratégia de investimento ótima $\varkappa^{*}=(\varkappa(0), \ldots, \varkappa(T-1))$ para os problemas PTE $\left(\sigma^{2}\right)$ e PTE $(\epsilon)$ é dada pela lei de controle (6.10), com $\lambda(T)=\frac{\ell(T)}{(1-2 b)}$ em (4.2) $e$

$$
\left\{\begin{array}{l}
\ell(T)=1 \text { e } \nu(T)=\sqrt{\frac{b}{2(1-2 b) \sigma^{2}}}, \text { para o problema PTE }\left(\sigma^{2}\right), \\
\ell(T)=\frac{\epsilon(1-2 b)}{b} \text { e } \nu(T)=1, \text { para o problema PTE }(\epsilon) .
\end{array}\right.
$$

Sob a política de investimento ótima $\varkappa^{*}$, a esperança e a variância da riqueza final e a fronteira eficiente de investimentos são, respectivamente, obtidas pelas expressões:

$$
\begin{gathered}
E\left(G^{\varkappa^{*}}(T)\right)=\frac{\ell(T) b}{\nu(T)(1-2 b)}, \\
\operatorname{Var}\left(G^{\varkappa^{*}}(T)\right)=\frac{\ell(T)^{2} b}{2 \nu(T)^{2}(1-2 b)}
\end{gathered}
$$

$e$

$$
\operatorname{Var}\left(G^{\varkappa^{*}}(T)\right)=\frac{(1-2 b)}{2 b} E\left(G^{\varkappa^{*}}(T)\right)^{2}
$$

com b dado por (5.4).

Prova. A prova segue diretamente dos Teoremas 5.3, 6.2 e 6.3 e dos Corolários 6.1 e 6.2 .

O próximo teorema expõe a solução para os problemas $P T E\left(\sigma^{2}\right)$ e $P T E(\epsilon)$ para o caso em que existe no universo de investimento um ativo livre de risco, como definido na Seção 5.4 . 
Teorema 6.5 Uma política ótima de investimentos $\varkappa^{*}=(\varkappa(0), \ldots, \varkappa(T-1))$ para os problemas PTE $\left(\sigma^{2}\right)$ e PTE $(\epsilon)$, considerando que o ativo $i=0$ é livre de risco, é dada pela lei de controle:

$$
\varkappa^{*}(t)=-\phi_{\theta(t)}(t)^{-1} \chi_{\theta(t)}(t)\left(\hat{A}(t) V(t)+\frac{\lambda(T)}{2 \rho(T) \vartheta(t+1)}\right),
$$

$\operatorname{com} \lambda(T)=\frac{\ell(T)}{(1-2 b)}$ em (4.2) $e$

$$
\left.\begin{array}{l}
\left.\begin{array}{l}
E\left(G^{\varkappa^{*}}(T)\right)=\epsilon, \nu(T)=\rho(T)=1, \\
\ell(T)=\frac{2 \pi(0)^{\prime} \mathcal{H}(0) \epsilon}{1-\pi(0)^{\prime} \mathcal{H}(0)},
\end{array}\right\} \text { para o problema PTE }(\epsilon), \\
\operatorname{Var}\left(G^{\varkappa^{*}}(T)\right)=\sigma^{2}, \ell(T)=1, \\
\rho(T)=\nu(T)=\frac{1}{2 \sigma} \sqrt{\frac{1-\pi(0)^{\prime} \mathcal{H}(0)}{\pi(0)^{\prime} \mathcal{H}(0)}},
\end{array}\right\} \text { para o problema PTE }\left(\sigma^{2}\right),
$$

$\nu(t)=\rho(t)=\ell(t)=0$ para $t \neq T$ e com $b$ dado por (5.33). Além disso, para a lei de controle ótima $\varkappa^{*}$, a esperança e a variância da riqueza final e a fronteira eficiente de investimentos são descritos, respectivamente, por:

$$
\begin{gathered}
E\left(G^{\varkappa^{*}}(T)\right)=\frac{\ell(T)\left(1-\pi(0)^{\prime} \mathcal{H}(0)\right)}{2 \nu(T) \pi(0)^{\prime} \mathcal{H}(0)}, \\
\operatorname{Var}\left(G^{\varkappa^{*}}(T)\right)=\frac{\ell(T)^{2}\left(1-\pi(0)^{\prime} \mathcal{H}(0)\right)}{4 \nu(T)^{2} \pi(0)^{\prime} \mathcal{H}(0)}
\end{gathered}
$$

$e$

$$
\operatorname{Var}\left(G^{\varkappa^{*}}(T)\right)=\frac{1-\pi(0)^{\prime} \mathcal{H}(0)}{\pi(0)^{\prime} \mathcal{H}(0)} E\left(G^{\varkappa^{*}}(T)\right)^{2} .
$$

Prova. A prova segue diretamente dos Teoremas 5.4 e 6.4 .

Comparando os Teoremas 6.4 e 6.5, observa-se que, para os problemas PTE $\left(\sigma^{2}\right)$ e PTE $(\epsilon)$ com o enfoque do TE, as diferenças entre as soluções quando o universo de investimento é formado apenas por ativos de risco e quando há um ativo livre de risco, se tornam mais sutis que as entre os Teoremas 5.3 e 5.4. Isto ocorre, como descrito na subseção anterior, pelo fato de que no enfoque do TE, o gestor de carteiras pode 
a qualquer momento neutralizar o risco, mesmo com o universo formado apenas por ativos de risco, bastando para isso replicar o benchmark. 


\section{Capítulo 7}

\section{Exemplos Numéricos}

Neste capítulo, os modelos apresentados são avaliados através de exemplos numéricos. Compara-se os resultados obtidos através dos modelos propostos neste trabalho, representados pelos problemas $P\left(\sigma^{2}\right), P M V\left(\sigma^{2}\right)$ e $P B C(\varrho, \psi)$, entre si e com aquele introduzido em Li e Ng (2000), que é similar ao problema $P\left(\sigma^{2}\right)$, desconsiderando-se a influência da cadeia de Markov. Os estudos são realizados com ativos brasileiros, aplicando-os ao caso em que todos os ativos são de risco e à situação em que no universo de investimento um dos ativos é livre de risco. Adicionalmente, são desenvolvidas simulações de Monte Carlo para verificar o comportamento das carteiras.

Também são realizadas simulações para os problemas de otimização de carteiras para superar um benchmark, representados pelo problema PMVTE $\left(\sigma^{2}\right)$. A fonte para todos os dados utilizados foi o sistema BLOOMBERG. Já para as simulações foi utilizado o sistema Matlab.

\subsection{Mercado apenas com Ativos de Risco}

Nesta seção serão comparados os resultados de quatro problemas. Os dois primeiros casos são o de maximizar a riqueza final, mantendo a variância do patrimônio final igual 
ou abaixo de um determinado valor. Estes dois problemas representam o problema $P\left(\sigma^{2}\right)$ e estão descritos como dois, pois em um será considerada a influência da cadeia de Markov sobre os parâmetros dos ativos e será denominado de $\mathrm{P}$ e o segundo, descrito também em Li e Ng (2000), não considera esta fonte de incerteza sobre os ativos e será chamado de $\mathrm{P}_{\mathrm{sm}}$. O terceiro modelo é aquele em que o investidor deseja maximizar a soma ponderada da riqueza em cada período, também com a restrição de manter a variância do patrimônio final igual ou abaixo de um determinado valor. Este modelo representa o problema $P M V\left(\sigma^{2}\right)$, e será identificado simplesmente por $P M V$. Para a ponderação da esperança da carteira do investidor $(E(V(t)))$, atribuiu-se o mesmo peso a todos os períodos, ou seja, $\alpha(t)=1$, para todo $t$. O último caso é o que considera a função objetivo como uma soma ponderada do portfólio ao longo do tempo, mas que possui restrições sobre a queda do valor da riqueza abaixo de um determinado valor $(\psi(t))$, com uma dada probabilidade máxima $(\varrho(t))$. Este problema representa o modelo $P B C(\varrho, \psi)$ e será identificado por PBC. Assim como em $P M V$, considerouse o mesmo peso $\alpha(t)=1$ para $E(V(t))$ na função objetivo. Também, para melhor comparar o resultado deste problema com o dos outros modelos, escolheu-se $\varrho(T)$ e $\psi(T)$, convenientemente, de forma que a restrição sobre a variância final do problema $P B C(\varrho, \psi)$ fosse a mesma que dos modelos anteriores.

Assumiu-se que o investidor em questão tenha uma unidade de riqueza e horizonte de investimento de quatro trimestres $(T=4)$. Como proxy do mercado foi utilizado o índice IBOVESPA. O retorno e a variância anual média deste índice no período de 2000 a 2006 foi de $\mu_{I B O V}=14,9 \%, \sigma_{I B O V}^{2}=0,085$.

Como restrição básica para os modelos a serem investigados nesta seção, determinase que o investidor não deseja que a variância de sua riqueza final seja superior à variância média do mercado, ou seja $\sigma^{2}(V(T)) \leq 0,085$. Além disso, no problema $P B C$, o investidor aceita que seu patrimônio se desvalorize para abaixo de $50 \%$ do valor inicial $(\psi(t)=0,5$, para $t=1, \ldots, T-1)$ nos períodos intermediários com uma probabilidade máxima de $10 \%(\varrho(t)=10 \%$, para $t=1, \ldots, T-1)$. 
Considerou-se a existência de duas tendências principais do mercado: positiva e negativa, ou seja, $\mathcal{M}=\{1,2\}$ respectivamente. Para identificar a tendência predominante em cada momento, utilizou-se uma média móvel de 63 dias de negócios (três meses) no período de 2000 a 2006 e o índice de mercado. Quando o valor diário do índice está acima da média móvel de 63 dias de negócios e cruza a média móvel para baixo, a tendência predominante muda de positiva para negativa. Quando o valor diário do índice está abaixo da média móvel de 63 dias de negócios e cruza a média móvel para cima, a tendência predominante muda de negativa para positiva.

Para estimar o retorno médio $\bar{\mu}(t, \theta(t))$ e a matriz de covariância $\bar{\sigma}^{2}(t, \theta(t))$ dos ativos, adotou-se um procedimento similar ao realizado em Zhang (2000). A partir dos preços de fechamento diários das ações no período de 2000 a 2006, obteve-se o vetor de retornos médios e a matriz de covariância trimestrais (63 dias de negócios) para cada modo de mercado. Estes vetores de retorno médio e as matrizes de covariância são utilizados como estimativas para implementação dos modelos apresentados neste trabalho. Para simplificar, assume-se que nos exercícios realizados o processo em multiperíodo é estacionário.

Para estimar o vetor de retorno médio e a matriz de covariância dos ativos a serem utilizados no modelo $\mathrm{P}_{\mathrm{sm}}$, utilizou-se todo o período como amostra (2000 - 2006) sem separá-lo em tendências.

Para determinar a tendência geral do mercado brasileiro escolheu-se o Índice da Bolsa de Valores de São Paulo (IBOVESPA) e como universo de ativos de risco, foram utilizadas todas as ações pertencentes a este índice. Na Figura 7.20 segue a lista dos ativos de risco com seus respectivos códigos Bloomberg e pesos no índice IBOVESPA.

Os vetores de retornos médios e as matrizes de covariâncias trimestrais destes ativos em todo o período de amostragem e para cada uma das respectivas tendências predominantes do mercado (positiva e negativa) são apresentados nas Tabelas 7.20 a 7.32 . Para melhorar a visualização das tabelas de covariâncias, elas foram apresentadas de forma 
segmentada em quatro partes, conforme a matriz exemplo abaixo:

$$
\left[\begin{array}{cc}
\text { Parte I } & \text { Parte II } \\
\text { Parte III } & \text { Parte IV }
\end{array}\right] \text {. }
$$

O número de amostras (dias de negócios) no período utilizado para estimação dos parâmetros retorno esperado e variância foi de 1.737 dias de negócios. Deste total, 1.066 foram dias em que a tendência predominante do mercado era positiva e em 671 dias a tendência negativa prevaleceu. Como os dias de tendência positiva representaram $61 \%$ do total de dias de negócios, escolheu-se como matriz de probabilidades de transição:

$$
P(t)=\left[\begin{array}{ll}
61 \% & 39 \% \\
61 \% & 39 \%
\end{array}\right]
$$

e como probabilidades de estado o vetor $\pi(t)=\left(\pi_{1}(t), \pi_{2}(t)\right)^{\prime}=(61 \%, 39 \%)^{\prime}$. Denotase como Caso Base (CB) a simulação com estes dados para a matriz de probabilidades de transição e vetor de probabilidades de estado.

Os resultados encontrados pela implementação dos modelos estão sumarizados na Figura 7.1 e nas Tabelas 7.1 a 7.4. A Figura 7.1 apresenta a fronteira eficiente resultante dos quatro modelos analisados. Cada curva corresponde às escolhas ótimas de risco (variância da riqueza final) e retorno (esperança da riqueza final) que cada modelo apresenta. Pelas fronteiras eficientes, nota-se que para o mesmo nível de risco os problemas com saltos superaram o problema Psm, que não está sujeito à influência da cadeia de Markov. Este fato destaca a vantagem dos modelo com saltos em relação àqueles sem esta propriedade, pois os primeiros conseguem se aproveitar melhor dos movimentos do mercado.

Para verificar o comportamento das carteiras geradas pelas otimizações, realizaramse simulações de Monte Carlo com 10.000 cenários para cada uma. A partir das simulações de Monte Carlo, extraiu-se a média e a variância dos patrimônios para cada período, identificados respectivamente como $\hat{E}(V(t))$ e $\widehat{\operatorname{Var}}(V(t))$. Durante as simu- 
lações também se quantificou o número de vezes $(N F(t))$ em que o patrimônio esteve abaixo do valor mínimo determinado $(\psi(t)<0,5)$, e consequentemente a probabilidade deste evento ocorrer, definida como $\widehat{\varrho}(t)=N F(t) / 10.000$. Portanto, é possível comparar os resultados teóricos dos modelos com aqueles provenientes da simulação de Monte Carlo.

As Tabelas 7.1 a 7.4 mostram os resultados tanto da otimização quanto da simulação de Monte Carlo para as carteiras ótimas que atendem à restrição sobre a variância final $\sigma^{2}(V(T)) \leq 0,085$ e, para o problema $P B C$, que atendem à restrição da probabilidade máxima de $10 \%(\varrho(t)=10 \%$, para $t=1, \ldots, T-1)$ de que o patrimônio se desvalorize para abaixo de $50 \%$ do valor inicial $(\psi(t)=0,5$, para $t=1, \ldots, T-1)$ nos períodos intermediários.

Apesar do modelo P apresentar um valor esperado para o patrimônio final $2 \%$ maior que o $P M V$, o valor esperado para a riqueza nos dois primeiros intervalos de tempo foi maior no segundo modelo. Isto ocorre porque o último modelo leva em conta os valores esperados da riqueza nos períodos intermediários. Portanto, um investidor que tenha um interesse maior por um determinado instante intermediário pode atingir melhor seu objetivo, utilizando o modelo $P M V$.

Observa-se que o modelo que apresentou o menor número de eventos em que o valor do portfólio caiu para abaixo de $50 \%$ do valor inicial foi o modelo $P B C$, o qual possui uma restrição sobre a probabilidade máxima deste fato ocorrer.

Em todos os modelos, o resultado da variância da carteira foi diferente entre a otimização e a simulação de Monte Carlo. Entretanto, a diferença entre os dois resultados foi menor no modelo $\mathrm{P}_{\mathrm{SM}}$.

Observa-se pela Tabela 7.17 que as carteiras geradas quando existe um ativo livre de risco são bem concentradas em determinados ativos e possuem posições vendidas com intensidade considerável.

Para verificar a dependência dos resultados a alterações nas probabilidades de transição e estado, foram realizadas mais duas simulações: a primeira considerando a matriz 


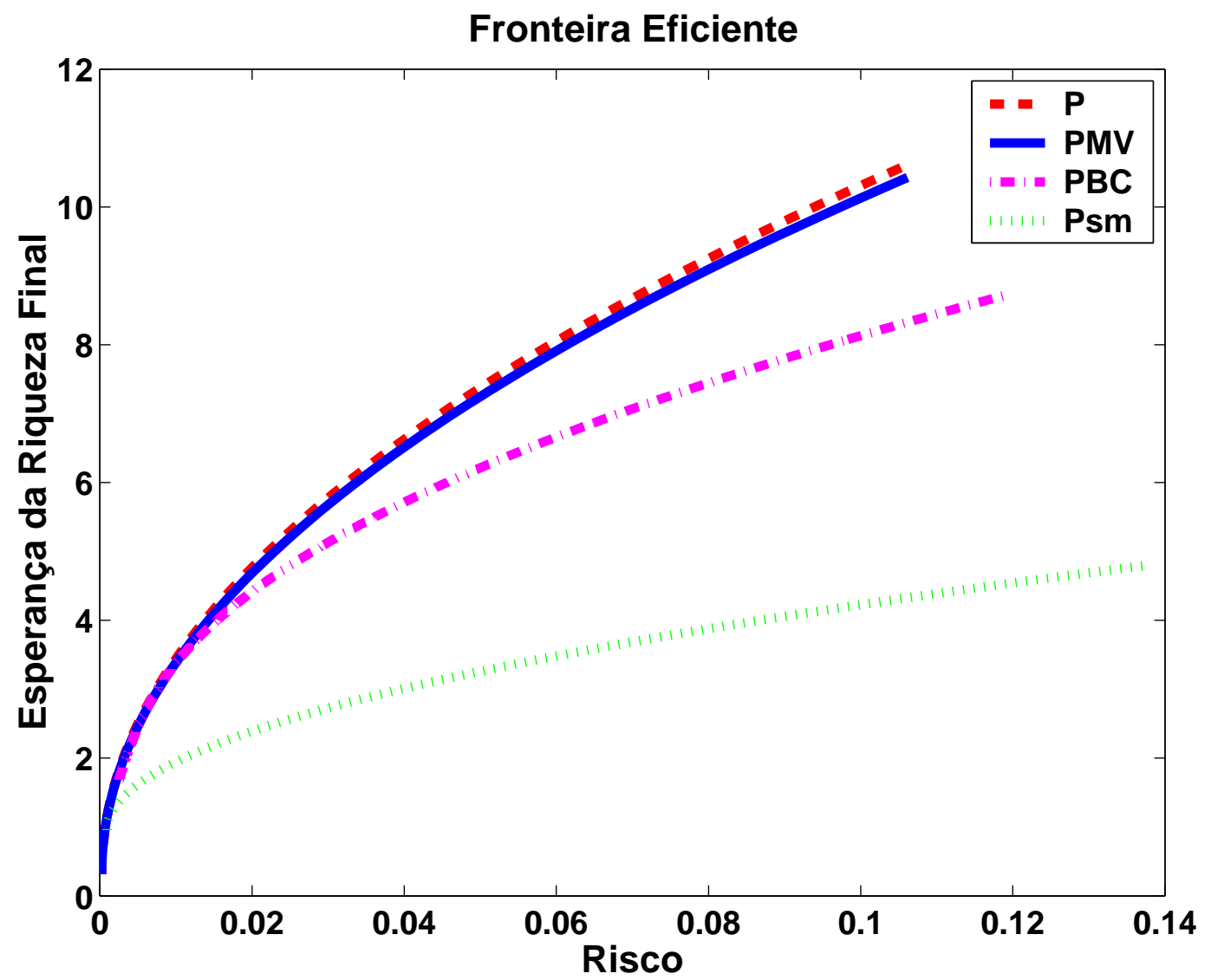

Figura 7.1: Fronteiras Eficientes (Esperança da Riqueza Final $x$ Variância do Patrimônio Final) - Mercado formado apenas por ativos de risco brasileiros.

Resultados do problema $\mathrm{P}$

\begin{tabular}{cccccccc}
\hline \hline Período $(\mathrm{t})$ & $E(V(t))$ & $\hat{E}(V(t))$ & $\operatorname{Var}(V(t))$ & $\widehat{\operatorname{Var}}(V(t))$ & $\varrho(t)$ & $N F(t)$ & $\widehat{\varrho}(t)$ \\
\hline \hline 1 & 7,97 & 7,83 & 7,34 & 15,07 & $13,1 \%$ & 382 & $3,8 \%$ \\
2 & 9,05 & 8,98 & 1,18 & 5,12 & $1,6 \%$ & 83 & $0,8 \%$ \\
3 & 9,34 & 9,33 & 0,22 & 1,89 & $0,3 \%$ & 20 & $0,2 \%$ \\
4 & 9,53 & 9,52 & 0,085 & 0,78 & $0,1 \%$ & 6 & $0,1 \%$ \\
\hline \hline Total & & & & & 491 & $4,9 \%$ \\
\hline \hline
\end{tabular}

Tabela 7.1: Resultados das simulações de Monte Carlo para o problema P 
Resultados do problema $P M V$

\begin{tabular}{cccccccc}
\hline \hline Período $(\mathrm{t})$ & $E(V(t))$ & $\hat{E}(V(t))$ & $\operatorname{Var}(V(t))$ & $\widehat{\operatorname{Var}}(V(t))$ & $\varrho(t)$ & $N F(t)$ & $\widehat{\varrho}(t)$ \\
\hline \hline 1 & 8,79 & 8,63 & 9,17 & 18,82 & $13,3 \%$ & 391 & $3,9 \%$ \\
2 & 9,14 & 9,09 & 1,3 & 5,92 & $1,7 \%$ & 89 & $0,9 \%$ \\
3 & 9,22 & 9,22 & 0,23 & 2,12 & $0,3 \%$ & 25 & $0,3 \%$ \\
4 & 9,36 & 9,36 & 0,085 & 0,87 & $0,1 \%$ & 9 & $0,1 \%$ \\
\hline \hline Total & & & & & 514 & $5,1 \%$ \\
\hline \hline
\end{tabular}

Tabela 7.2: Resultados das simulações de Monte Carlo para o problema $P M V$

Resultados do problema $P B C$

\begin{tabular}{cccccccc}
\hline \hline Período $(\mathrm{t})$ & $E(V(t))$ & $\hat{E}(V(t))$ & $\operatorname{Var}(V(t))$ & $\widehat{\operatorname{Var}}(V(t))$ & $\varrho(t)$ & $N F(t)$ & $\widehat{\varrho}(t)$ \\
\hline \hline 1 & 3,23 & 3,19 & 0,75 & 1,54 & $10,0 \%$ & 243 & $2,4 \%$ \\
2 & 7,12 & 7,00 & 2,34 & 5,30 & $5,4 \%$ & 125 & $1,3 \%$ \\
3 & 7,51 & 7,48 & 0,36 & 1,80 & $0,7 \%$ & 29 & $0,3 \%$ \\
4 & 7,62 & 7,62 & 0,085 & 0,67 & $0,2 \%$ & 9 & $0,1 \%$ \\
\hline \hline Total & & & & & & 406 & $4,1 \%$ \\
\hline \hline
\end{tabular}

Tabela 7.3: Resultados das simulações de Monte Carlo para o problema $P B C$

Resultados do problema $\mathrm{P}_{\mathrm{SM}}$

\begin{tabular}{cccccccc}
\hline \hline Período $(\mathrm{t})$ & $E(V(t))$ & $\hat{E}(V(t))$ & $\operatorname{Var}(V(t))$ & $\widehat{\operatorname{Var}}(V(t))$ & $\varrho(t)$ & $N F(t)$ & $\widehat{\varrho}(t)$ \\
\hline \hline 1 & 2,97 & 2,98 & 1,67 & 1,80 & $27,2 \%$ & 310 & $3,1 \%$ \\
2 & 3,62 & 3,63 & 0,68 & 0,77 & $7,0 \%$ & 82 & $0,8 \%$ \\
3 & 3,86 & 3,86 & 0,24 & 0,27 & $2,1 \%$ & 13 & $0,1 \%$ \\
4 & 3,97 & 3,97 & 0,085 & 0,10 & $0,7 \%$ & 3 & $0,0 \%$ \\
\hline \hline Total & & & & & & 408 & $4,1 \%$ \\
\hline \hline
\end{tabular}

Tabela 7.4: Resultados das simulações de Monte Carlo para o problema $\mathrm{P}_{\mathrm{SM}}$

de probabilidade como

$$
P(t)=\left[\begin{array}{ll}
40 \% & 60 \% \\
40 \% & 60 \%
\end{array}\right]
$$

e como probabilidades de estado o vetor $\pi(t)=(40 \%, 60 \%)^{\prime}$, denotada de Caso Negativo $(\mathrm{CN})$, e a segunda considerando a matriz de probabilidade como

$$
P(t)=\left[\begin{array}{ll}
80 \% & 20 \% \\
80 \% & 20 \%
\end{array}\right]
$$


e como probabilidades de estado o vetor $\pi(t)=(80 \%, 20 \%)^{\prime}$, denominada de Caso Positivo (CP). Os resultados destas duas simulações estão sumarizados nas Figuras 7.2 e 7.3 e nas Tabelas 7.5 a 7.7 e 7.8 a 7.10 , respectivamente.

A alteração da matriz de probabilidades de transição não altera as conclusões gerais descritas para a primeira simulação. Entretanto, percebe-se que com a elevação da probabilidade de que a tendência positiva seja a predominante, o valor esperado para a riqueza final em cada instante se eleva.

Observa-se também que com a elevação da probabilidade da tendência predominante ser a positiva, os resultados para o modelo $P B C$ se aproximam dos modelos $\mathrm{P}$ e $P M V$. Entretanto, esta aproximação provoca um custo referente a um maior número de eventos em que o valor da carteira cai abaixo de $50 \%$ do valor inicial.

\subsection{Mercado no qual Existe um Ativo Livre de Risco}

Nesta seção, realiza-se a primeira simulação da seção anterior, incluindo no universo de investimento um ativo livre de risco. O objetivo é o mesmo da seção anterior, otimizar os quatro problemas ( $\mathrm{P}, P M V, P B C$ e $\left.\mathrm{P}_{\mathrm{sm}}\right)$, considerando que o investidor em questão tem uma unidade de riqueza e horizonte de investimento de quatro trimestres $(T=4)$. Como restrição básica para os modelos, determina-se que o investidor não deseja que a variância de sua riqueza final seja superior à variância média do mercado, ou seja $\sigma^{2}(V(T)) \leq 0,085$. Além disso, no problema $P B C$, o investidor aceita que seu patrimônio se desvalorize para abaixo de $50 \%$ do valor inicial $(\psi(t)=0,5$, para $t=1, \ldots, T-1)$ nos períodos intermediários com uma probabilidade máxima de $10 \%$ $(\varrho(t)=10 \%$, para $t=1, \ldots, T-1)$.

Para a simulação dos modelos, utilizou-se os mesmos ativos e condições, vetores de retorno e matrizes de covariância, do exemplo somente com ativos de risco. Incluiu-se apenas o ativo livre de risco, para o qual foi selecionado o CDI (Certificado de Depósito Interbancário). Apesar do CDI variar ao longo do tempo, seguindo as definições da taxa 
Fronteira Eficiente

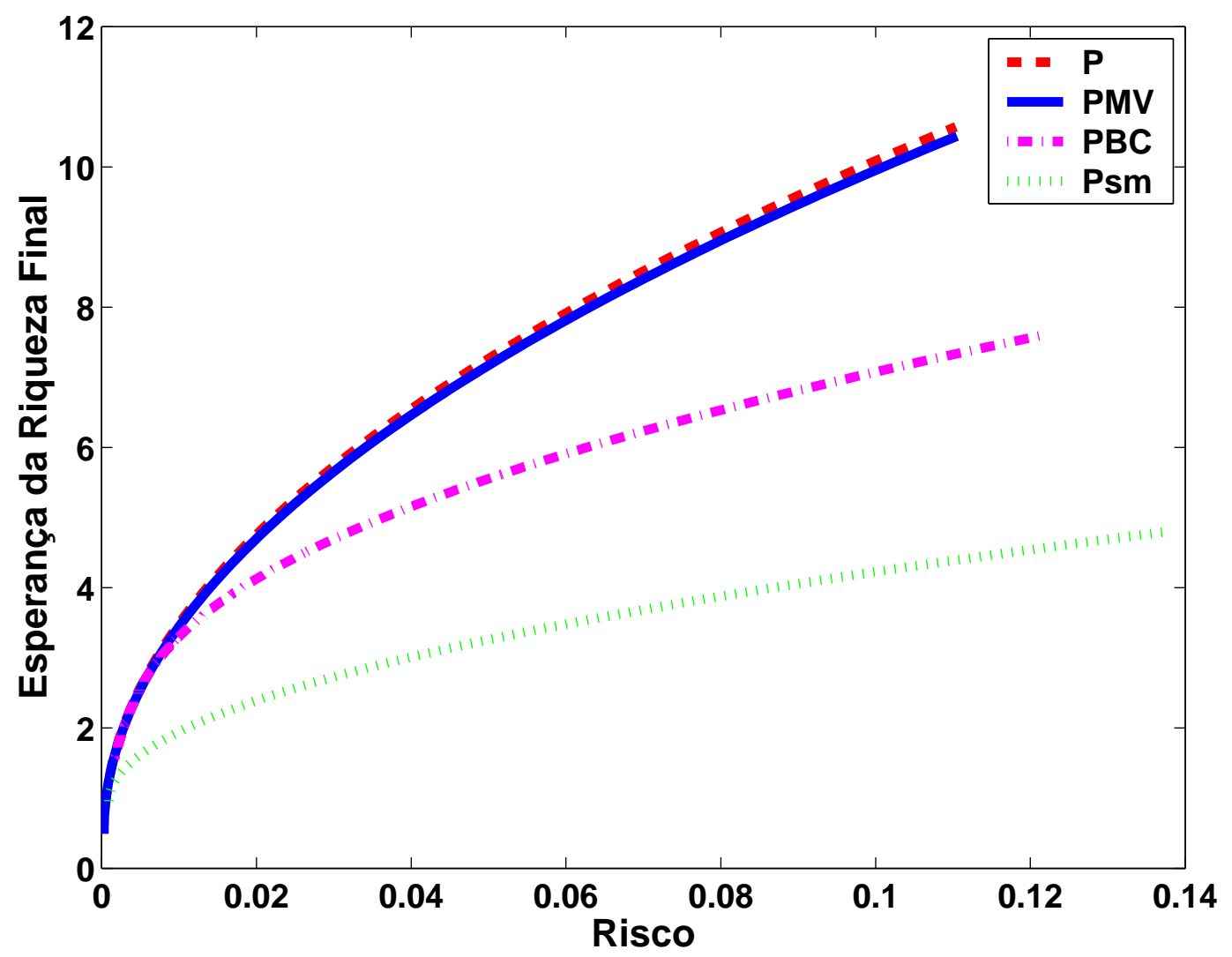

Figura 7.2: Fronteiras Eficientes (Esperança da Riqueza Final $x$ Variância do Patrimônio Final) - Mercado formado apenas com ativos de risco brasileiros e com vetor de probabilidade de estado $\pi(t)=(40 \%, 60 \%)^{\prime}$.

Resultados do problema $\mathrm{P}$

\begin{tabular}{cccccccc}
\hline \hline Período $(\mathrm{t})$ & $E(V(t))$ & $\hat{E}(V(t))$ & $\operatorname{Var}(V(t))$ & $\widehat{\operatorname{Var}}(V(t))$ & $\varrho(t)$ & $N F(t)$ & $\widehat{\varrho}(t)$ \\
\hline \hline 1 & 7,83 & 7,84 & 8,26 & 15,12 & $15,4 \%$ & 383 & $3,8 \%$ \\
2 & 8,95 & 8,94 & 1,5 & 5,09 & $2,1 \%$ & 84 & $0,8 \%$ \\
3 & 9,21 & 9,22 & 0,28 & 1,86 & $0,4 \%$ & 20 & $0,2 \%$ \\
4 & 9,33 & 9,34 & 0,085 & 0,77 & $0,1 \%$ & 7 & $0,1 \%$ \\
\hline \hline Total & & & & & 494 & $4,9 \%$ \\
\hline
\end{tabular}

Tabela 7.5: Resultados das simulações de Monte Carlo para o problema $\mathrm{P}$ 
Resultados do problema $P M V$

\begin{tabular}{cccccccc}
\hline \hline Período $(\mathrm{t})$ & $E(V(t))$ & $\hat{E}(V(t))$ & $\operatorname{Var}(V(t))$ & $\widehat{\operatorname{Var}}(V(t))$ & $\varrho(t)$ & $N F(t)$ & $\widehat{\varrho}(t)$ \\
\hline \hline 1 & 8,41 & 8,43 & 9,73 & 17,82 & $15,5 \%$ & 388 & $3,9 \%$ \\
2 & 9,04 & 9,03 & 1,58 & 5,66 & $2,2 \%$ & 90 & $0,9 \%$ \\
3 & 9,13 & 9,14 & 0,28 & 2,03 & $0,4 \%$ & 23 & $0,2 \%$ \\
4 & 9,21 & 9,22 & 0,085 & 0,84 & $0,1 \%$ & 8 & $0,1 \%$ \\
\hline \hline Total & & & & & 509 & $5,1 \%$ \\
\hline \hline
\end{tabular}

Tabela 7.6: Resultados das simulações de Monte Carlo para o problema $P M V$

Resultados do problema $P B C$

\begin{tabular}{cccccccc}
\hline \hline Período $(\mathrm{t})$ & $E(V(t))$ & $\hat{E}(V(t))$ & $\operatorname{Var}(V(t))$ & $\widehat{\operatorname{Var}}(V(t))$ & $\varrho(t)$ & $N F(t)$ & $\widehat{\varrho}(t)$ \\
\hline \hline 1 & 2,53 & 2,54 & 0,41 & 0,76 & $10,0 \%$ & 174 & $1,7 \%$ \\
2 & 6,20 & 6,18 & 2,42 & 4,62 & $7,5 \%$ & 138 & $1,4 \%$ \\
3 & 6,59 & 6,59 & 0,41 & 0,41 & $1,1 \%$ & 33 & $0,3 \%$ \\
4 & 6,67 & 6,68 & 0,085 & 0,085 & $0,2 \%$ & 12 & $0,1 \%$ \\
\hline \hline Total & & & & & & 357 & $3,6 \%$ \\
\hline \hline
\end{tabular}

Tabela 7.7: Resultados das simulações de Monte Carlo para o problema $P B C$

básica de juros determinada pelo Banco Central, ela foi considerada como constante e igual a sua média no período de amostra (2000 a 2006). Portanto, considera-se o ativo livre de risco como o ativo $i=0 \operatorname{com} r_{f}(t)=18,15 \%$ e covariância nula com os outros ativos de risco.

A matriz de probabilidades de transição e o vetor de probabilidades de estado utilizados são os mesmos para o Caso Base da seção anterior, ou seja:

$$
P(t)=\left[\begin{array}{ll}
61 \% & 39 \% \\
61 \% & 39 \%
\end{array}\right], \quad \pi(t)=\left[\begin{array}{l}
61 \% \\
39 \%
\end{array}\right]
$$

Os resultados encontrados pela implementação dos modelos estão sumarizados na Figura 7.1 e nas Tabelas 7.11 a 7.14.

Observa-se que a inclusão do ativo livre de risco permite a elevação da esperança dos valores do portfólio em todos os períodos e para todos os modelos. Entretanto, isto ocorre conjuntamente com a elevação do risco nos períodos intermediários.

Pelas fronteiras eficientes, nota-se que para o mesmo nível de risco os problemas com 
Fronteira Eficiente

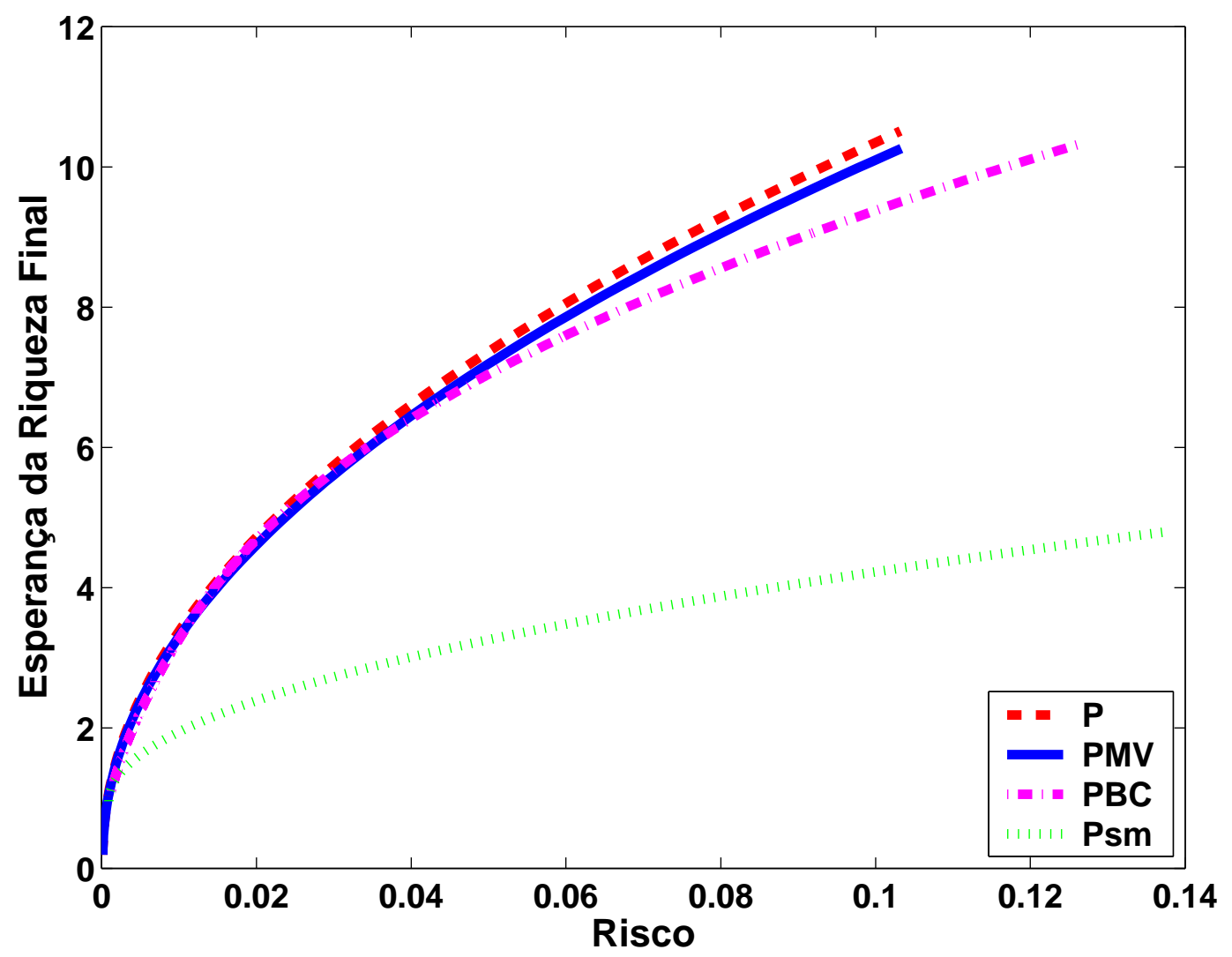

Figura 7.3: Fronteiras Eficientes (Esperança da Riqueza Final $x$ Variância do Patrimônio Final) - Mercado formado apenas com ativos de risco brasileiros e com vetor de probabilidade de estado $\pi(t)=(80 \%, 20 \%)^{\prime}$.

Resultados do problema $\mathrm{P}$

\begin{tabular}{cccccccc}
\hline \hline Período $(\mathrm{t})$ & $E(V(t))$ & $\hat{E}(V(t))$ & $\operatorname{Var}(V(t))$ & $\widehat{\operatorname{Var}}(V(t))$ & $\varrho(t)$ & $N F(t)$ & $\widehat{\varrho}(t)$ \\
\hline \hline 1 & 7,93 & 7,66 & 6,17 & 14,32 & $11,2 \%$ & 380 & $3,8 \%$ \\
2 & 8,95 & 8,85 & 0,88 & 4,92 & $1,2 \%$ & 82 & $0,8 \%$ \\
3 & 9,29 & 9,27 & 0,17 & 1,83 & $0,2 \%$ & 20 & $0,2 \%$ \\
4 & 9,56 & 9,54 & 0,085 & 0,77 & $0,1 \%$ & 6 & $0,1 \%$ \\
\hline \hline Total & & & & & 488 & $4,9 \%$ \\
\hline
\end{tabular}

Tabela 7.8: Resultados das simulações de Monte Carlo para o problema $P B C$ 
Resultados do problema $P M V$

\begin{tabular}{cccccccc}
\hline \hline Período $(\mathrm{t})$ & $E(V(t))$ & $\hat{E}(V(t))$ & $\operatorname{Var}(V(t))$ & $\widehat{\operatorname{Var}}(V(t))$ & $\varrho(t)$ & $N F(t)$ & $\widehat{\varrho}(t)$ \\
\hline \hline 1 & 9,12 & 8,8 & 8,46 & 16,93 & $11,4 \%$ & 391 & $3,9 \%$ \\
2 & 9,06 & 8,99 & 1,08 & 6,12 & $1,5 \%$ & 93 & $0,9 \%$ \\
3 & 9,12 & 9,11 & 0,19 & 2,17 & $0,3 \%$ & 24 & $0,2 \%$ \\
4 & 9,33 & 9,31 & 0,085 & 0,90 & $0,1 \%$ & 9 & $0,1 \%$ \\
\hline \hline Total & \multicolumn{7}{c}{} \\
\hline \hline
\end{tabular}

Tabela 7.9: Resultados das simulações de Monte Carlo para o problema $P M V$

Resultados do problema $P B C$

\begin{tabular}{cccccccc}
\hline \hline Período $(\mathrm{t})$ & $E(V(t))$ & $\hat{E}(V(t))$ & $\operatorname{Var}(V(t))$ & $\widehat{\operatorname{Var}}(V(t))$ & $\varrho(t)$ & $N F(t)$ & $\widehat{\varrho}(t)$ \\
\hline \hline 1 & 4,83 & 4,68 & 1,88 & 4,38 & $10,0 \%$ & 320 & $3,2 \%$ \\
2 & 8,25 & 8,06 & 1,66 & 5,13 & $2,8 \%$ & 91 & $0,9 \%$ \\
3 & 8,58 & 8,53 & 0,25 & 1,82 & $0,4 \%$ & 25 & $0,3 \%$ \\
4 & 8,77 & 8,75 & 0,085 & 0,70 & $0,1 \%$ & 6 & $0,1 \%$ \\
\hline \hline Total & & & & & & 442 & $4,4 \%$ \\
\hline \hline
\end{tabular}

Tabela 7.10: Resultados das simulações de Monte Carlo para o problema $P B C$

saltos Markovianos superaram o problema Psm de forma ainda mais pronunciada que na primeira simulação que considerava apenas ativos de risco. Percebe-se uma redução da probabilidade de o portfólio cair abaixo de $50 \%$ do valor inicial, tanto pela inequação de Tchebycheff, quanto pela simulação de Monte Carlo. Os modelos P, PMV e PBC apresentaram resultados similares, embora, o modelo $\mathrm{P}$ ainda apresente o maior valor esperado para a riqueza final.

Observa-se pela Tabela 7.18 que as carteiras geradas quando existe um ativo livre de risco são bem mais concentradas em determinados ativos e possuem posições vendidas com intensidade maior que no caso em que os ativos são apenas de risco. Este fato ocorre principalmente devido a alavancagem no ativo livre de risco. 
Fronteira Eficiente

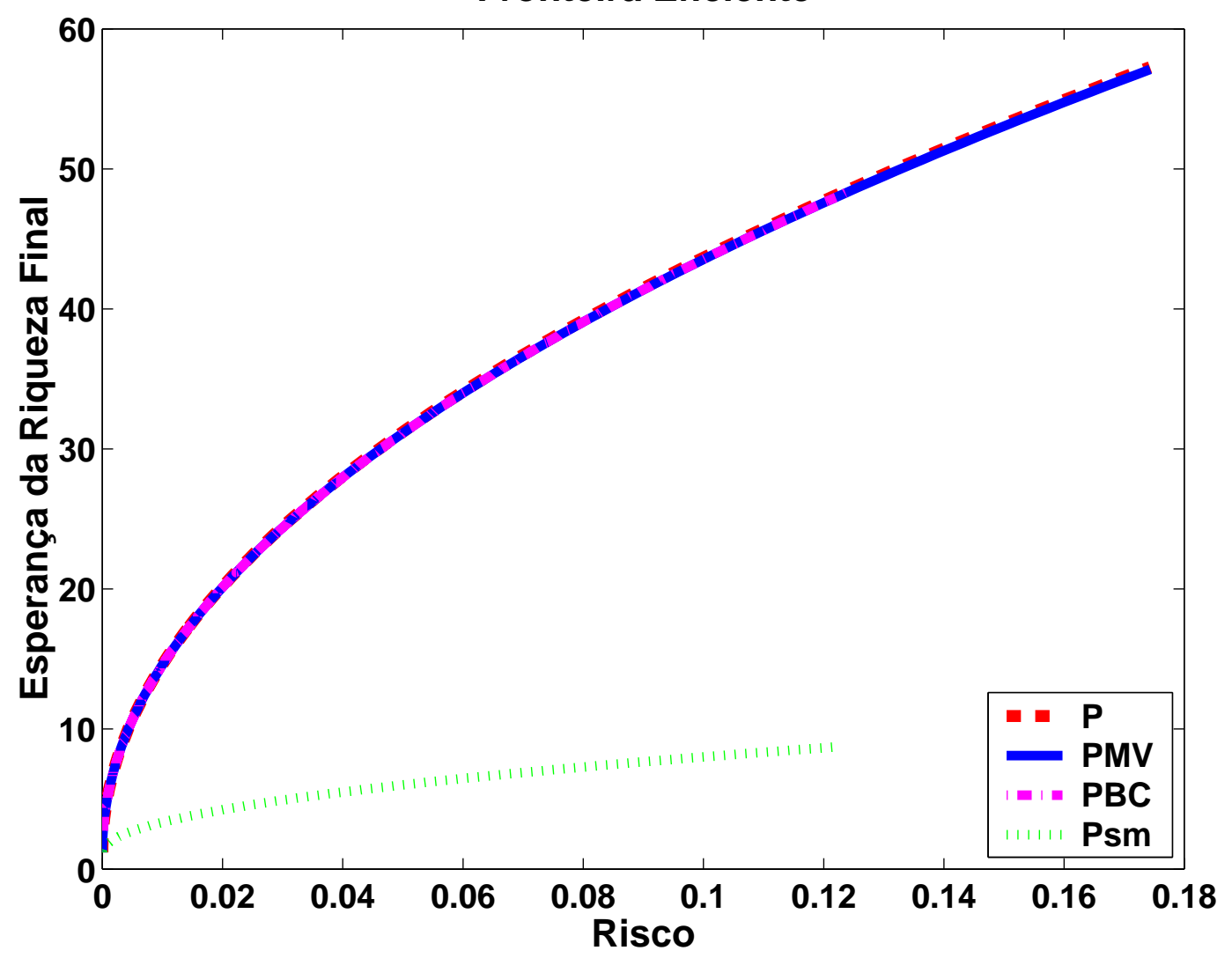

Figura 7.4: Fronteiras Eficientes (Esperança da Riqueza Final $x$ Variância do Patrimônio Final) - Mercado formado por ativos de risco e um livre de risco.

Resultados do problema $\mathrm{P}$

\begin{tabular}{cccccccc}
\hline \hline Período $(\mathrm{t})$ & $E(V(t))$ & $\hat{E}(V(t))$ & $\operatorname{Var}(V(t))$ & $\widehat{\operatorname{Var}}(V(t))$ & $\varrho(t)$ & $N F(t)$ & $\widehat{\varrho}(t)$ \\
\hline \hline 1 & 32,62 & 32,72 & 94,18 & 132,26 & $9,1 \%$ & 26 & $0,3 \%$ \\
2 & 36,85 & 36,95 & 9,59 & 18,25 & $0,7 \%$ & 0 & 0 \\
3 & 38,68 & 38,70 & 0,91 & 2,50 & $0,1 \%$ & 0 & 0 \\
4 & 40,35 & 40,35 & 0,085 & 0,34 & $0,0 \%$ & 0 & 0 \\
\hline \hline Total & & & & & 26 & $0,3 \%$ \\
\hline \hline
\end{tabular}

Tabela 7.11: Resultados das simulações de Monte Carlo para o problema P 
Resultados do problema $P M V$

\begin{tabular}{cccccccc}
\hline \hline Período $(\mathrm{t})$ & $E(V(t))$ & $\hat{E}(V(t))$ & $\operatorname{Var}(V(t))$ & $\widehat{\operatorname{Var}}(V(t))$ & $\varrho(t)$ & $N F(t)$ & $\widehat{\varrho}(t)$ \\
\hline \hline 1 & 33,24 & 33,34 & 97,91 & 137,51 & $9,1 \%$ & 26 & $0,3 \%$ \\
2 & 36,88 & 36,97 & 9,65 & 18,49 & $0,7 \%$ & 0 & 0 \\
3 & 38,58 & 38,60 & 0,91 & 2,53 & $0,1 \%$ & 0 & 0 \\
4 & 40,23 & 40,23 & 0,085 & 0,34 & $0,0 \%$ & 0 & 0 \\
\hline \hline Total & \multicolumn{7}{c}{} \\
\hline \hline
\end{tabular}

Tabela 7.12: Resultados das simulações de Monte Carlo para o problema $P M V$

Resultados do problema $P B C$

\begin{tabular}{cccccccc}
\hline \hline Período $(\mathrm{t})$ & $E(V(t))$ & $\hat{E}(V(t))$ & $\operatorname{Var}(V(t))$ & $\widehat{\operatorname{Var}}(V(t))$ & $\varrho(t)$ & $N F(t)$ & $\widehat{\varrho}(t)$ \\
\hline \hline 1 & 33,24 & 33,34 & 97,91 & 137,51 & $9,1 \%$ & 26 & $0,3 \%$ \\
2 & 36,88 & 36,97 & 9,65 & 18,49 & $0,7 \%$ & 0 & 0 \\
3 & 38,58 & 38,60 & 0,91 & 2,53 & $0,1 \%$ & 0 & 0 \\
4 & 40,23 & 40,23 & 0,085 & 0,34 & $0,0 \%$ & 0 & 0 \\
\hline \hline Total & & & & 26 & $0,3 \%$ \\
\hline \hline
\end{tabular}

Tabela 7.13: Resultados das simulações de Monte Carlo para o problema $P B C$

Tabela XIV

Resultados do problema Psm

\begin{tabular}{cccccccc}
\hline \hline Período $(\mathrm{t})$ & $E(V(t))$ & $\hat{E}(V(t))$ & $\operatorname{Var}(V(t))$ & $\widehat{\operatorname{Var}}(V(t))$ & $\varrho(t)$ & $N F(t)$ & $\widehat{\varrho}(t)$ \\
\hline \hline 1 & 5,41 & 5,43 & 5,23 & 5,73 & $21,7 \%$ & 186 & $1,9 \%$ \\
2 & 6,62 & 6,65 & 1,49 & 1,68 & $4,0 \%$ & 16 & $0,2 \%$ \\
3 & 7,13 & 7,13 & 0,36 & 0,42 & $0,8 \%$ & 2 & $0,0 \%$ \\
4 & 7,48 & 7,49 & 0,085 & 0,10 & $0,2 \%$ & 0 & 0 \\
\hline \hline Total & & & & & 204 & $2,0 \%$ \\
\hline \hline
\end{tabular}

Tabela 7.14: Resultados das simulações de Monte Carlo para o problema Psm

\subsection{Otimização de Carteira para Superar um Benchmark}

Nesta seção são realizadas as simulações dos modelo de seleção de carteiras para superar um benchmark. Em geral, os administradores de carteira profissionais têm como objetivo de investimento obter uma rentabilidade superior a um determinado benchmark, mantendo a variância do tracking error abaixo de um determinado limite máximo, fornecido pelos seus clientes. Para investidores institucionais (fundos de pensão), 
geralmente este limite máximo é de $12 \%$ a.a. para o desvio padrão do tracking error, que resulta em uma variância do tracking error de $1,44 \cdot 10^{-2}\left(0,12^{2}\right)$.

Portanto, considera-se o problema que um gestor de recursos tem de otimizar seu portfólio em um horizonte de investimentos de quatro trimestres $(T=4)$, de forma maximizar a soma ponderada do tracking error ao longo do tempo, mantendo a variância do TE limitada a $1,44 \cdot 10^{-2}$ em cada período. Este problema foi denominado na Seção 6.2.1 de PMVTE $\left(\sigma^{2}\right)$.

Assume-se que o benchmark escolhido pelo investidor seja o IBOVESPA e o universo de investimento seja formado apenas pelas ações que compõem este índice.

Realizaram-se duas simulações: uma que considera que os parâmetros estão sujeitos a saltos Markovianos PMVTE, e outra, chamada de PMVTE $E_{s m}$, cujos parâmetros não estão sujeitos a saltos. Os dados utilizados para estas simulações são os mesmos utilizados na Seção 7.1 para o Caso Base.

Os resultados encontrados pela implementação destas duas simulações estão sumarizados na Figura 7.5 e nas Tabelas 7.15 e 7.16. Novamente, os resultados do modelo com saltos supera os do modelo sem esta propriedade, ilustrando o benefício desta característica. Observa-se que a rentabilidade final alcançada pelos modelos com o enfoque do tracking error é bem inferior à de qualquer dos modelos dos exemplos anteriores sem este enfoque do tracking error. Portanto, ilustrando que de fato os gestores possuem um menor limite de risco em sua tarefa de seleção de portfólios do que a simples variância de mercado, como utilizada nos exemplos anteriores.

Observa-se também pela Tabela 7.19 que as carteiras geradas neste exemplo tem os pesos nos ativos de forma menos concentrada que a observada nos exemplos anteriores. Embora também possua posições vendidas em ativos, estas também são em menor intensidade. Portanto, se aproximando mais de uma carteira real. 


\begin{tabular}{ccc}
\multicolumn{3}{c}{ Resultados do modelo PMVTE } \\
\hline \hline Período $(\mathrm{t})$ & $E(G(t))$ & $\operatorname{Var}(G(t))$ \\
\hline \hline 1 & 0,31 & $1,44 \cdot 10^{-2}$ \\
2 & 0,36 & $1,44 \cdot 10^{-2}$ \\
3 & 0,67 & $1,44 \cdot 10^{-2}$ \\
4 & 0,96 & $1,44 \cdot 10^{-2}$ \\
\hline \hline
\end{tabular}

Tabela 7.15: Resultados das simulações para o problema PMVTE

\begin{tabular}{ccc}
\multicolumn{3}{c}{ Resultados do modelo $P M V T E_{s m}$} \\
\hline \hline Período $(\mathrm{t})$ & $E(G(t))$ & $\operatorname{Var}(G(t))$ \\
\hline \hline 1 & 0,18 & $1,44 \cdot 10^{-2}$ \\
2 & 0,34 & $1,44 \cdot 10^{-2}$ \\
3 & 0,49 & $1,44 \cdot 10^{-2}$ \\
4 & 0,65 & $1,44 \cdot 10^{-2}$ \\
\hline \hline
\end{tabular}

Tabela 7.16: Resultados das simulações para o problema $P M V T E_{s m}$ 


\section{Fronteira Eficiente}

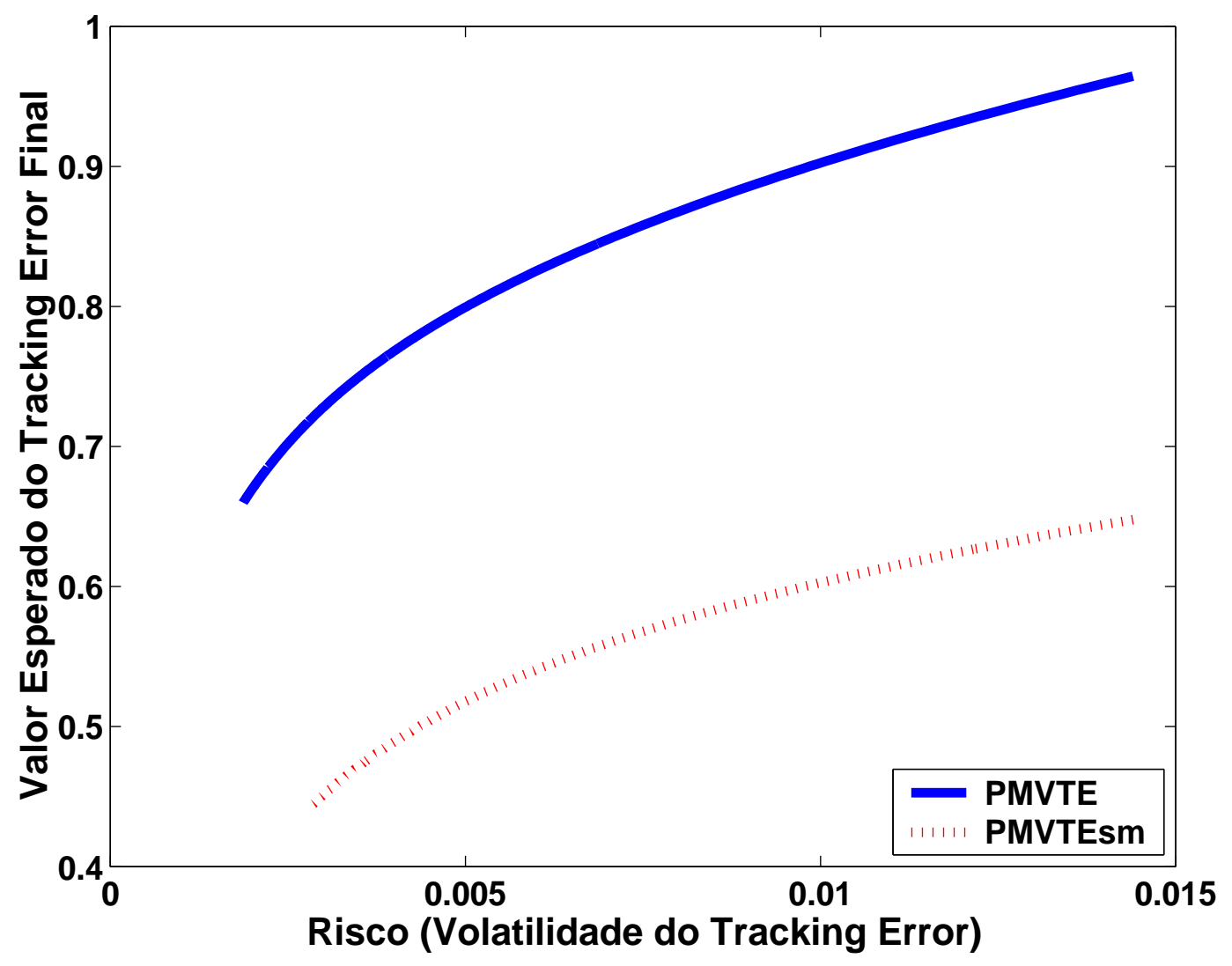

Figura 7.5: Fronteiras Eficientes (Valor Esperado do Tracking Error Final $x$ Variância do Tracking Error Final) para os problemas de seleção de carteiras para superar um benchmark, com controle sobre a volatilidade do tracking error (variância do tracking error). 


\begin{tabular}{|c|c|c|c|c|c|c|c|}
\hline & & & & & & & \\
\hline & $\begin{array}{l}\text { Tendêcia } \\
\text { Positiva }\end{array}$ & $\begin{array}{c}\text { Tendência } \\
\text { Negativa }\end{array}$ & $\begin{array}{l}\text { Tendêcia } \\
\text { Positiva }\end{array}$ & $\begin{array}{c}\text { Tendência } \\
\text { Negativa }\end{array}$ & $\begin{array}{l}\text { Tendêcia } \\
\text { Positiva }\end{array}$ & $\begin{array}{c}\text { Tendência } \\
\text { Negativa }\end{array}$ & Psm \\
\hline PETR4 & $119,8 \%$ & $-353,1 \%$ & $133,9 \%$ & $-394,6 \%$ & $38,3 \%$ & $-113,1 \%$ & $16,2 \%$ \\
\hline VALE5 & $-96,8 \%$ & $240,5 \%$ & $-108,3 \%$ & $268,8 \%$ & $-30,5 \%$ & $77,0 \%$ & $10,1 \%$ \\
\hline BBDC4 & $288,6 \%$ & $-148,1 \%$ & $322,6 \%$ & $-165,4 \%$ & $91,6 \%$ & $-47,4 \%$ & $125,8 \%$ \\
\hline USIM5 & $269,1 \%$ & $-496,7 \%$ & $301,0 \%$ & $-555,1 \%$ & $84,5 \%$ & $-159,0 \%$ & $50,3 \%$ \\
\hline ITAU4 & $106,6 \%$ & $-49,2 \%$ & $119,2 \%$ & $-55,0 \%$ & $33,7 \%$ & $-15,4 \%$ & $-10,3 \%$ \\
\hline TNLP4 & $-321,1 \%$ & $-8,4 \%$ & $-359,1 \%$ & $-9,3 \%$ & $-101,1 \%$ & $-3,2 \%$ & $-143,7 \%$ \\
\hline GGBR4 & $2,4 \%$ & $325,3 \%$ & $2,7 \%$ & $363,6 \%$ & $0,9 \%$ & $104,0 \%$ & $58,8 \%$ \\
\hline PETR3 & $222,5 \%$ & $-20,5 \%$ & $248,6 \%$ & $-23,0 \%$ & $71,3 \%$ & $-6,5 \%$ & $106,2 \%$ \\
\hline VALE3 & $138,5 \%$ & $-50,8 \%$ & $155,0 \%$ & $-56,7 \%$ & $43,5 \%$ & $-16,4 \%$ & $155,0 \%$ \\
\hline CSNA3 & $186,1 \%$ & $-189,0 \%$ & $208,0 \%$ & $-211,2 \%$ & $59,3 \%$ & $-60,7 \%$ & $-3,5 \%$ \\
\hline UBBR11 & $-583,6 \%$ & $482,7 \%$ & $-652,3 \%$ & $539,4 \%$ & $-185,8 \%$ & $154,5 \%$ & $-167,2 \%$ \\
\hline CMIG4 & $-18,9 \%$ & $-79,0 \%$ & $-21,1 \%$ & $-88,3 \%$ & $-6,2 \%$ & $-25,1 \%$ & $6,2 \%$ \\
\hline ELET6 & $-21,7 \%$ & $205,9 \%$ & $-24,2 \%$ & $230,0 \%$ & $-6,9 \%$ & $66,0 \%$ & $10,5 \%$ \\
\hline ITSA4 & $195,3 \%$ & $286,5 \%$ & $218,4 \%$ & $320,2 \%$ & $61,9 \%$ & $91,7 \%$ & $207,1 \%$ \\
\hline BBAS3 & $108,2 \%$ & $305,4 \%$ & $121,0 \%$ & $341,3 \%$ & $34,4 \%$ & $97,5 \%$ & $109,8 \%$ \\
\hline TAMM4 & $95,1 \%$ & $32,9 \%$ & $106,4 \%$ & $36,6 \%$ & $30,2 \%$ & $11,5 \%$ & $89,2 \%$ \\
\hline NETC4 & $-74,0 \%$ & $-228,0 \%$ & $-82,7 \%$ & $-254,8 \%$ & $-23,4 \%$ & $-72,9 \%$ & $-137,5 \%$ \\
\hline ALLL11 & $110,0 \%$ & $231,3 \%$ & $122,8 \%$ & $258,5 \%$ & $35,8 \%$ & $73,5 \%$ & $166,4 \%$ \\
\hline TNLP3 & $89,8 \%$ & $-5,7 \%$ & $100,5 \%$ & $-6,5 \%$ & $27,6 \%$ & $-1,3 \%$ & $-10,0 \%$ \\
\hline ELET3 & $-172,8 \%$ & $34,1 \%$ & $-193,1 \%$ & $38,1 \%$ & $-55,1 \%$ & $10,7 \%$ & $-97,6 \%$ \\
\hline CSAN3 & $88,7 \%$ & $22,1 \%$ & $98,9 \%$ & $25,1 \%$ & $29,4 \%$ & $5,0 \%$ & $-14,3 \%$ \\
\hline GOLL4 & $-79,0 \%$ & $269,2 \%$ & $-88,5 \%$ & $300,6 \%$ & $-24,0 \%$ & $87,6 \%$ & $-15,1 \%$ \\
\hline BRAP4 & $172,2 \%$ & $-279,9 \%$ & $192,5 \%$ & $-312,8 \%$ & $54,7 \%$ & $-89,7 \%$ & $-42,0 \%$ \\
\hline VIVO4 & $-257,0 \%$ & $-186,3 \%$ & $-287,3 \%$ & $-208,2 \%$ & $-81,8 \%$ & $-59,6 \%$ & $-183,8 \%$ \\
\hline AMBV4 & $-36,3 \%$ & $521,5 \%$ & $-40,8 \%$ & $582,7 \%$ & $-10,4 \%$ & $167,0 \%$ & $120,3 \%$ \\
\hline BRKM5 & $177,2 \%$ & $-398,7 \%$ & $198,1 \%$ & $-445,6 \%$ & $56,3 \%$ & $-127,6 \%$ & $-46,4 \%$ \\
\hline NATU3 & $65,8 \%$ & $209,5 \%$ & $73,2 \%$ & $234,1 \%$ & $23,2 \%$ & $67,2 \%$ & $126,2 \%$ \\
\hline CPLE6 & $-120,3 \%$ & $186,2 \%$ & $-134,4 \%$ & $208,1 \%$ & $-38,9 \%$ & $59,4 \%$ & $-60,3 \%$ \\
\hline SUBA3 & $53,5 \%$ & $-52,6 \%$ & $59,7 \%$ & $-58,8 \%$ & $17,9 \%$ & $-16,3 \%$ & $33,4 \%$ \\
\hline PRGA3 & $67,7 \%$ & $70,3 \%$ & $75,7 \%$ & $78,6 \%$ & $21,2 \%$ & $22,5 \%$ & $27,6 \%$ \\
\hline GOAU4 & $278,7 \%$ & $-131,8 \%$ & $311,5 \%$ & $-147,2 \%$ & $88,7 \%$ & $-42,2 \%$ & $135,6 \%$ \\
\hline CCRO3 & $288,2 \%$ & $-306,6 \%$ & $322,1 \%$ & $-342,6 \%$ & $92,0 \%$ & $-98,0 \%$ & $116,0 \%$ \\
\hline TCSL4 & $-63,6 \%$ & $15,1 \%$ & $-71,0 \%$ & $16,9 \%$ & $-20,3 \%$ & $4,8 \%$ & $-40,2 \%$ \\
\hline CYRE3 & $277,7 \%$ & $-435,3 \%$ & $310,3 \%$ & $-486,1 \%$ & $88,9 \%$ & $-141,5 \%$ & $151,7 \%$ \\
\hline ARCZ6 & $-126,1 \%$ & $382,9 \%$ & $-141,1 \%$ & $428,0 \%$ & $-39,5 \%$ & $122,2 \%$ & $15,1 \%$ \\
\hline BRTO4 & $153,0 \%$ & $-325,7 \%$ & $171,0 \%$ & $-363,9 \%$ & $48,5 \%$ & $-104,4 \%$ & $-42,3 \%$ \\
\hline EMBR3 & $85,7 \%$ & $-12,5 \%$ & $95,7 \%$ & $-14,0 \%$ & $28,0 \%$ & $-3,8 \%$ & $19,3 \%$ \\
\hline SDIA4 & $59,1 \%$ & $-20,2 \%$ & $66,2 \%$ & $-22,6 \%$ & $18,4 \%$ & $-6,3 \%$ & $66,3 \%$ \\
\hline CPFE3 & $-288,0 \%$ & $201,6 \%$ & $-322,6 \%$ & $224,9 \%$ & $-87,8 \%$ & $67,0 \%$ & $-129,7 \%$ \\
\hline PCAR4 & $84,4 \%$ & $-586,8 \%$ & $94,2 \%$ & $-655,8 \%$ & $27,3 \%$ & $-187,5 \%$ & $-93,6 \%$ \\
\hline VCPA4 & $-27,6 \%$ & $-147,7 \%$ & $-30,9 \%$ & $-165,1 \%$ & $-8,2 \%$ & $-46,5 \%$ & $-7,7 \%$ \\
\hline CESP6 & $-235,6 \%$ & $2562,5 \%$ & $-265,6 \%$ & $2858,6 \%$ & $-61,7 \%$ & $848,5 \%$ & $150,0 \%$ \\
\hline SBSP3 & $28,1 \%$ & $-293,2 \%$ & $31,3 \%$ & $-327,6 \%$ & $9,4 \%$ & $-94,1 \%$ & $-92,7 \%$ \\
\hline LREN3 & $71,4 \%$ & $-109,0 \%$ & $79,8 \%$ & $-121,8 \%$ & $22,7 \%$ & $-34,8 \%$ & $37,2 \%$ \\
\hline ELPL6 & $-1406,7 \%$ & $-2167,1 \%$ & $-1579,4 \%$ & $-2428,0 \%$ & $-407,5 \%$ & $-657,2 \%$ & $-870,4 \%$ \\
\hline BRTP4 & $-234,0 \%$ & $5,6 \%$ & $-261,6 \%$ & $6,3 \%$ & $-74,1 \%$ & $1,6 \%$ & $-104,6 \%$ \\
\hline KLBN4 & $-36,3 \%$ & $113,3 \%$ & $-40,7 \%$ & $126,6 \%$ & $-11,1 \%$ & $36,3 \%$ & $19,4 \%$ \\
\hline TMAR5 & $-43,7 \%$ & $-32,3 \%$ & $-48,9 \%$ & $-36,1 \%$ & $-13,6 \%$ & $-10,2 \%$ & $-8,9 \%$ \\
\hline TCSL3 & $184,9 \%$ & $-159,2 \%$ & $206,8 \%$ & $-177,9 \%$ & $58,6 \%$ & $-50,9 \%$ & $73,0 \%$ \\
\hline CRUZ3 & $-25,8 \%$ & $322,8 \%$ & $-28,8 \%$ & $360,6 \%$ & $-8,1 \%$ & $103,8 \%$ & $72,0 \%$ \\
\hline LIGT3 & $-53,9 \%$ & $-107,0 \%$ & $-60,6 \%$ & $-119,8 \%$ & $-15,6 \%$ & $-33,1 \%$ & $-156,8 \%$ \\
\hline BRTP3 & $0,9 \%$ & $22,7 \%$ & $1,1 \%$ & $25,4 \%$ & $-0,2 \%$ & $6,9 \%$ & $40,7 \%$ \\
\hline TRPL4 & $155,9 \%$ & $166,8 \%$ & $174,3 \%$ & $186,5 \%$ & $49,4 \%$ & $53,2 \%$ & $89,8 \%$ \\
\hline CLsC6 & $-86,7 \%$ & $71,5 \%$ & $-96,9 \%$ & $79,9 \%$ & $-27,6 \%$ & $23,0 \%$ & $-44,8 \%$ \\
\hline TMCP4 & $114,3 \%$ & $-121,0 \%$ & $127,8 \%$ & $-135,2 \%$ & $36,3 \%$ & $-38,5 \%$ & $40,2 \%$ \\
\hline TLPP4 & $-37,1 \%$ & $256,0 \%$ & $-41,5 \%$ & $286,0 \%$ & $-11,1 \%$ & $82,6 \%$ & $-1,5 \%$ \\
\hline PTIP4 & $-80,7 \%$ & $38,3 \%$ & $-90,3 \%$ & $42,8 \%$ & $-25,7 \%$ & $12,5 \%$ & $-7,8 \%$ \\
\hline CGAS5 & $238,3 \%$ & $10,3 \%$ & $266,3 \%$ & $11,5 \%$ & $75,9 \%$ & $3,3 \%$ & $132,9 \%$ \\
\hline ACES4 & $49,5 \%$ & $8,5 \%$ & $55,2 \%$ & $9,6 \%$ & $16,4 \%$ & $2,6 \%$ & $54,1 \%$ \\
\hline
\end{tabular}

Tabela 7.17: Carteiras para o Primeiro Período no Problema de Otimização de Carteiras com Ativos de Risco apenas. 


\begin{tabular}{|c|c|c|c|c|c|c|c|}
\hline & & & & IV & & & \\
\hline & $\begin{array}{l}\text { Tendêcia } \\
\text { Positiva }\end{array}$ & $\begin{array}{c}\text { Tendência } \\
\text { Negativa }\end{array}$ & $\begin{array}{l}\text { Tendêcia } \\
\text { Positiva }\end{array}$ & $\begin{array}{c}\text { Tendência } \\
\text { Negativa }\end{array}$ & $\begin{array}{l}\text { Tendêcia } \\
\text { Positiva }\end{array}$ & $\begin{array}{c}\text { Tendência } \\
\text { Negativa }\end{array}$ & Psm \\
\hline CDI & $4590,2 \%$ & $-915,3 \%$ & $4678,4 \%$ & $-906,8 \%$ & $4678,4 \%$ & $-906,8 \%$ & $5485,3 \%$ \\
\hline PETR4 & $492,1 \%$ & $-485,3 \%$ & $501,8 \%$ & $-494,9 \%$ & $501,8 \%$ & $-494,9 \%$ & $14,7 \%$ \\
\hline VALE5 & $-425,9 \%$ & $-10,4 \%$ & $-434,3 \%$ & $-10,4 \%$ & $-434,3 \%$ & $-10,4 \%$ & $12,3 \%$ \\
\hline BBDC4 & $1225,1 \%$ & $7,3 \%$ & $1249,2 \%$ & $7,3 \%$ & $1249,2 \%$ & $7,3 \%$ & $162,2 \%$ \\
\hline USIM5 & $1211,1 \%$ & $21,9 \%$ & $1234,9 \%$ & $21,9 \%$ & $1234,9 \%$ & $21,9 \%$ & $109,6 \%$ \\
\hline ITAU4 & $462,7 \%$ & $9,5 \%$ & $471,8 \%$ & $9,5 \%$ & $471,8 \%$ & $9,5 \%$ & $-9,4 \%$ \\
\hline TNLP4 & $-1420,1 \%$ & $-12,1 \%$ & $-1448,0 \%$ & $-12,1 \%$ & $-1448,0 \%$ & $-12,1 \%$ & $-230,6 \%$ \\
\hline GGBR4 & $-1,3 \%$ & $-17,8 \%$ & $-1,3 \%$ & $-17,8 \%$ & $-1,3 \%$ & $-17,8 \%$ & $90,3 \%$ \\
\hline PETR3 & $899,8 \%$ & $3,2 \%$ & $917,4 \%$ & $3,2 \%$ & $917,4 \%$ & $3,2 \%$ & $120,0 \%$ \\
\hline VALE3 & $624,2 \%$ & $-1,6 \%$ & $636,4 \%$ & $-1,6 \%$ & $636,4 \%$ & $-1,6 \%$ & $232,9 \%$ \\
\hline CSNA3 & $773,2 \%$ & $4,6 \%$ & $788,4 \%$ & $4,6 \%$ & $788,4 \%$ & $4,6 \%$ & $-2,7 \%$ \\
\hline UBBR11 & $-2436,1 \%$ & $-21,9 \%$ & $-2484,0 \%$ & $-21,9 \%$ & $-2484,0 \%$ & $-21,9 \%$ & $-214,8 \%$ \\
\hline CMIG4 & $-64,9 \%$ & $7,7 \%$ & $-66,2 \%$ & $7,7 \%$ & $-66,2 \%$ & $7,7 \%$ & $5,6 \%$ \\
\hline ELET6 & $-91,8 \%$ & $-6,7 \%$ & $-93,6 \%$ & $-6,7 \%$ & $-93,6 \%$ & $-6,7 \%$ & $20,5 \%$ \\
\hline ITSA4 & $839,1 \%$ & $-14,7 \%$ & $855,6 \%$ & $-14,7 \%$ & $855,6 \%$ & $-14,7 \%$ & $300,3 \%$ \\
\hline BBAS3 & $456,0 \%$ & $-18,7 \%$ & $464,9 \%$ & $-18,7 \%$ & $464,9 \%$ & $-18,7 \%$ & $161,7 \%$ \\
\hline TAMM4 & $401,8 \%$ & $19,9 \%$ & $409,7 \%$ & $19,9 \%$ & $409,7 \%$ & $19,9 \%$ & $120,9 \%$ \\
\hline NETC4 & $-318,3 \%$ & $12,6 \%$ & $-324,5 \%$ & $12,6 \%$ & $-324,5 \%$ & $12,6 \%$ & $-195,3 \%$ \\
\hline ALLL11 & $402,7 \%$ & $-22,0 \%$ & $410,6 \%$ & $-22,0 \%$ & $410,6 \%$ & $-22,0 \%$ & $186,8 \%$ \\
\hline TNLP3 & $447,0 \%$ & $10,9 \%$ & $455,7 \%$ & $11,0 \%$ & $455,7 \%$ & $11,0 \%$ & $33,5 \%$ \\
\hline ELET3 & $-713,5 \%$ & $-5,7 \%$ & $-727,5 \%$ & $-5,7 \%$ & $-727,5 \%$ & $-5,7 \%$ & $-116,8 \%$ \\
\hline CSAN3 & $286,6 \%$ & $-48,4 \%$ & $292,2 \%$ & $-48,4 \%$ & $292,2 \%$ & $-48,4 \%$ & $-29,9 \%$ \\
\hline GOLL4 & $-413,6 \%$ & $18,9 \%$ & $-421,7 \%$ & $18,9 \%$ & $-421,7 \%$ & $18,9 \%$ & $-117,2 \%$ \\
\hline BRAP4 & $730,8 \%$ & $9,4 \%$ & $745,2 \%$ & $9,4 \%$ & $745,2 \%$ & $9,4 \%$ & $-54,3 \%$ \\
\hline VIVO4 & $-1077,2 \%$ & $9,4 \%$ & $-1098,4 \%$ & $9,4 \%$ & $-1098,4 \%$ & $9,4 \%$ & $-245,1 \%$ \\
\hline AMBV4 & $-231,8 \%$ & $-22,6 \%$ & $-236,3 \%$ & $-22,6 \%$ & $-236,3 \%$ & $-22,6 \%$ & $112,2 \%$ \\
\hline BRKM5 & $747,4 \%$ & $19,7 \%$ & $762,1 \%$ & $19,7 \%$ & $762,1 \%$ & $19,7 \%$ & $-56,1 \%$ \\
\hline NATU3 & $113,1 \%$ & $-6,1 \%$ & $115,3 \%$ & $-6,0 \%$ & $115,3 \%$ & $-6,0 \%$ & $49,4 \%$ \\
\hline CPLE6 & $-459,3 \%$ & $-14,2 \%$ & $-468,3 \%$ & $-14,2 \%$ & $-468,3 \%$ & $-14,2 \%$ & $-36,9 \%$ \\
\hline SUBA3 & $162,1 \%$ & $13,6 \%$ & $165,3 \%$ & $13,6 \%$ & $165,3 \%$ & $13,6 \%$ & $24,7 \%$ \\
\hline PRGA3 & $310,8 \%$ & $-3,5 \%$ & $316,9 \%$ & $-3,5 \%$ & $316,9 \%$ & $-3,5 \%$ & $54,2 \%$ \\
\hline GOAU4 & $1164,9 \%$ & $4,7 \%$ & $1187,8 \%$ & $4,7 \%$ & $1187,8 \%$ & $4,7 \%$ & $178,6 \%$ \\
\hline CCRO3 & $1183,8 \%$ & $16,8 \%$ & $1207,1 \%$ & $16,8 \%$ & $1207,1 \%$ & $16,8 \%$ & $134,7 \%$ \\
\hline TCSL4 & $-262,1 \%$ & $-0,9 \%$ & $-267,3 \%$ & $-0,9 \%$ & $-267,3 \%$ & $-0,9 \%$ & $-47,8 \%$ \\
\hline CYRE3 & $1122,9 \%$ & $-27,8 \%$ & $1145,0 \%$ & $-27,9 \%$ & $1145,0 \%$ & $-27,9 \%$ & $225,1 \%$ \\
\hline ARCZ6 & $-571,2 \%$ & $-25,8 \%$ & $-582,4 \%$ & $-25,8 \%$ & $-582,4 \%$ & $-25,8 \%$ & $7,6 \%$ \\
\hline BRTO4 & $656,4 \%$ & $10,5 \%$ & $669,3 \%$ & $10,5 \%$ & $669,3 \%$ & $10,5 \%$ & $-36,6 \%$ \\
\hline EMBR3 & $304,8 \%$ & $5,7 \%$ & $310,8 \%$ & $5,7 \%$ & $310,8 \%$ & $5,7 \%$ & $-20,6 \%$ \\
\hline SDIA4 & $278,4 \%$ & $4,5 \%$ & $283,9 \%$ & $4,5 \%$ & $283,9 \%$ & $4,5 \%$ & $104,9 \%$ \\
\hline CPFE3 & $-1485,3 \%$ & $45,3 \%$ & $-1514,5 \%$ & $45,3 \%$ & $-1514,5 \%$ & $45,3 \%$ & $-434,8 \%$ \\
\hline PCAR4 & $318,3 \%$ & $35,0 \%$ & $324,5 \%$ & $35,1 \%$ & $324,5 \%$ & $35,1 \%$ & $-169,5 \%$ \\
\hline VCPA4 & $-156,7 \%$ & $24,0 \%$ & $-159,8 \%$ & $24,0 \%$ & $-159,8 \%$ & $24,0 \%$ & $-65,2 \%$ \\
\hline CESP6 & $-1943,9 \%$ & $521,2 \%$ & $-1982,0 \%$ & $522,0 \%$ & $-1982,0 \%$ & $522,0 \%$ & $-1131,3 \%$ \\
\hline SBSP3 & $83,0 \%$ & $9,2 \%$ & $84,6 \%$ & $9,2 \%$ & $84,6 \%$ & $9,2 \%$ & $-150,7 \%$ \\
\hline LREN3 & $298,1 \%$ & $10,4 \%$ & $303,9 \%$ & $10,6 \%$ & $303,9 \%$ & $10,6 \%$ & $49,6 \%$ \\
\hline ELPL6 & $-8787,9 \%$ & $911,7 \%$ & $-8960,5 \%$ & $912,0 \%$ & $-8960,5 \%$ & $912,0 \%$ & $-4583,5 \%$ \\
\hline BRTP4 & $-1006,4 \%$ & $-5,4 \%$ & $-1026,2 \%$ & $-5,4 \%$ & $-1026,2 \%$ & $-5,4 \%$ & $-147,9 \%$ \\
\hline KLBN4 & $-182,2 \%$ & $-10,0 \%$ & $-185,8 \%$ & $-10,2 \%$ & $-185,8 \%$ & $-10,2 \%$ & $6,7 \%$ \\
\hline TMAR5 & $-203,2 \%$ & $4,5 \%$ & $-207,2 \%$ & $4,5 \%$ & $-207,2 \%$ & $4,5 \%$ & $-33,7 \%$ \\
\hline TCSL3 & $789,2 \%$ & $8,5 \%$ & $804,7 \%$ & $8,5 \%$ & $804,7 \%$ & $8,5 \%$ & $100,0 \%$ \\
\hline CRUZ3 & $-112,7 \%$ & $-4,3 \%$ & $-115,0 \%$ & $-4,3 \%$ & $-115,0 \%$ & $-4,3 \%$ & $83,4 \%$ \\
\hline LIGT3 & $-341,6 \%$ & $31,0 \%$ & $-348,3 \%$ & $31,0 \%$ & $-348,3 \%$ & $31,0 \%$ & $-293,6 \%$ \\
\hline BRTP3 & $41,2 \%$ & $-7,9 \%$ & $42,0 \%$ & $-7,9 \%$ & $42,0 \%$ & $-7,9 \%$ & $92,9 \%$ \\
\hline TRPL4 & $669,6 \%$ & $-12,1 \%$ & $682,7 \%$ & $-12,1 \%$ & $682,7 \%$ & $-12,1 \%$ & $143,1 \%$ \\
\hline CLSC6 & $-365,1 \%$ & $-1,5 \%$ & $-372,2 \%$ & $-1,4 \%$ & $-372,2 \%$ & $-1,4 \%$ & $-62,4 \%$ \\
\hline TMCP4 & $482,4 \%$ & $10,1 \%$ & $491,9 \%$ & $10,1 \%$ & $491,9 \%$ & $10,1 \%$ & $38,7 \%$ \\
\hline TLPP4 & $-207,5 \%$ & $3,2 \%$ & $-211,6 \%$ & $3,2 \%$ & $-211,6 \%$ & $3,2 \%$ & $-60,1 \%$ \\
\hline PTIP4 & $-339,6 \%$ & $2,9 \%$ & $-346,2 \%$ & $2,9 \%$ & $-346,2 \%$ & $2,9 \%$ & $-20,1 \%$ \\
\hline CGAS5 & $989,7 \%$ & $-1,2 \%$ & $1009,2 \%$ & $-1,2 \%$ & $1009,2 \%$ & $-1,2 \%$ & $166,8 \%$ \\
\hline ACES4 & $160,7 \%$ & $-4,0 \%$ & $163,8 \%$ & $-4,0 \%$ & $163,8 \%$ & $-4,0 \%$ & $37,8 \%$ \\
\hline
\end{tabular}

Tabela 7.18: Carteiras para o Primeiro Período no Problema de Otimização de Carteiras com um Ativo Livre de Risco. 


\begin{tabular}{|c|c|c|c|}
\hline & & & \\
\hline & $\begin{array}{l}\text { Tendêcia } \\
\text { Positiva }\end{array}$ & $\begin{array}{c}\text { Tendência } \\
\text { Negativa }\end{array}$ & PMVTEsm \\
\hline PETR4 & $19,7 \%$ & $-15,6 \%$ & $1,5 \%$ \\
\hline VALE5 & $4,9 \%$ & $10,6 \%$ & $-2,6 \%$ \\
\hline BBDC4 & $16,9 \%$ & $-6,5 \%$ & $-33,3 \%$ \\
\hline USIM5 & $16,2 \%$ & $-22,0 \%$ & $-14,3 \%$ \\
\hline ITAU4 & $7,9 \%$ & $-2,2 \%$ & $2,6 \%$ \\
\hline TNLP4 & $-11,3 \%$ & $-0,3 \%$ & $39,1 \%$ \\
\hline GGBR4 & $3,0 \%$ & $14,4 \%$ & $-15,9 \%$ \\
\hline PETR3 & $12,4 \%$ & $-0,9 \%$ & $-27,7 \%$ \\
\hline VALE3 & $8,7 \%$ & $-2,2 \%$ & $-41,8 \%$ \\
\hline CSNA3 & $10,7 \%$ & $-8,3 \%$ & $0,9 \%$ \\
\hline UBBR11 & $-23,9 \%$ & $21,3 \%$ & $44,2 \%$ \\
\hline CMIG4 & $1,1 \%$ & $-3,5 \%$ & $-1,6 \%$ \\
\hline ELET6 & $0,9 \%$ & $9,1 \%$ & $-2,9 \%$ \\
\hline ITSA4 & $10,6 \%$ & $12,7 \%$ & $-55,6 \%$ \\
\hline BBAS3 & $6,7 \%$ & $13,5 \%$ & $-29,5 \%$ \\
\hline TAMM4 & $6,0 \%$ & $1,4 \%$ & $-23,7 \%$ \\
\hline NETC4 & $-1,6 \%$ & $-10,1 \%$ & $36,8 \%$ \\
\hline ALLL11 & $6,5 \%$ & $10,3 \%$ & $-43,4 \%$ \\
\hline TNLP3 & $5,7 \%$ & $-0,3 \%$ & $1,6 \%$ \\
\hline ELET3 & $-6,1 \%$ & $1,5 \%$ & $25,6 \%$ \\
\hline CSAN3 & $5,2 \%$ & $1,1 \%$ & $4,1 \%$ \\
\hline GOLL4 & $-2,2 \%$ & $11,8 \%$ & $6,2 \%$ \\
\hline BRAP4 & $9,1 \%$ & $-12,4 \%$ & $11,1 \%$ \\
\hline VIVO4 & $-10,0 \%$ & $-8,2 \%$ & $48,8 \%$ \\
\hline AMBV4 & $-0,3 \%$ & $23,0 \%$ & $-30,9 \%$ \\
\hline BRKM5 & $9,3 \%$ & $-17,6 \%$ & $12,2 \%$ \\
\hline NATU3 & $4,1 \%$ & $9,3 \%$ & $-30,8 \%$ \\
\hline CPLE6 & $-4,0 \%$ & $8,2 \%$ & $15,0 \%$ \\
\hline SUBA3 & $3,5 \%$ & $-2,4 \%$ & $-8,4 \%$ \\
\hline PRGA3 & $4,2 \%$ & $3,1 \%$ & $-7,7 \%$ \\
\hline GOAU4 & $13,5 \%$ & $-5,8 \%$ & $-36,0 \%$ \\
\hline CCRO3 & $13,9 \%$ & $-13,6 \%$ & $-30,3 \%$ \\
\hline TCSL4 & $-1,8 \%$ & $0,7 \%$ & $10,5 \%$ \\
\hline CYRE3 & $13,3 \%$ & $-19,1 \%$ & $-40,8 \%$ \\
\hline ARCZ6 & $-4,6 \%$ & $17,0 \%$ & $-3,7 \%$ \\
\hline BRTO4 & $7,8 \%$ & $-14,4 \%$ & $10,8 \%$ \\
\hline EMBR3 & $4,7 \%$ & $-0,6 \%$ & $-4,1 \%$ \\
\hline SDIA4 & $3,6 \%$ & $-0,9 \%$ & $-18,0 \%$ \\
\hline CPFE3 & $-12,1 \%$ & $8,8 \%$ & $40,5 \%$ \\
\hline PCAR4 & $4,6 \%$ & $-26,0 \%$ & $25,9 \%$ \\
\hline VCPA4 & $-0,4 \%$ & $-6,6 \%$ & $3,3 \%$ \\
\hline CESP6 & $-10,5 \%$ & $111,5 \%$ & $-9,3 \%$ \\
\hline SBSP3 & $2,0 \%$ & $-13,0 \%$ & $25,2 \%$ \\
\hline LREN3 & $4,0 \%$ & $-4,8 \%$ & $-9,9 \%$ \\
\hline ELPL6 & $-64,3 \%$ & $-98,2 \%$ & $309,6 \%$ \\
\hline BRTP4 & $-9,7 \%$ & $0,3 \%$ & $28,0 \%$ \\
\hline KLBN4 & $-1,1 \%$ & $5,0 \%$ & $-4,7 \%$ \\
\hline TMAR5 & $-1,4 \%$ & $-1,4 \%$ & $2,9 \%$ \\
\hline TCSL3 & $8,7 \%$ & $-7,0 \%$ & $-19,4 \%$ \\
\hline CRUZ3 & $-0,6 \%$ & $14,2 \%$ & $-18,8 \%$ \\
\hline LIGT3 & $-2,0 \%$ & $-4,8 \%$ & $43,6 \%$ \\
\hline BRTP3 & $0,6 \%$ & $1,0 \%$ & $-11,7 \%$ \\
\hline TRPL4 & $7,4 \%$ & $7,4 \%$ & $-24,4 \%$ \\
\hline CLSC6 & $-3,4 \%$ & $3,2 \%$ & $12,0 \%$ \\
\hline TMCP4 & $5,4 \%$ & $-5,4 \%$ & $-10,3 \%$ \\
\hline TLPP4 & $-1,3 \%$ & $11,3 \%$ & $1,7 \%$ \\
\hline PTIP4 & $-3,2 \%$ & $1,7 \%$ & $2,3 \%$ \\
\hline CGAS5 & $10,8 \%$ & $0,5 \%$ & $-35,1 \%$ \\
\hline ACES4 & $2,3 \%$ & $0,4 \%$ & $-13,6 \%$ \\
\hline
\end{tabular}

Tabela 7.19: Carteiras para o Primeiro Período no Problema de Otimização de Carteira para Superar um Benchmark. 


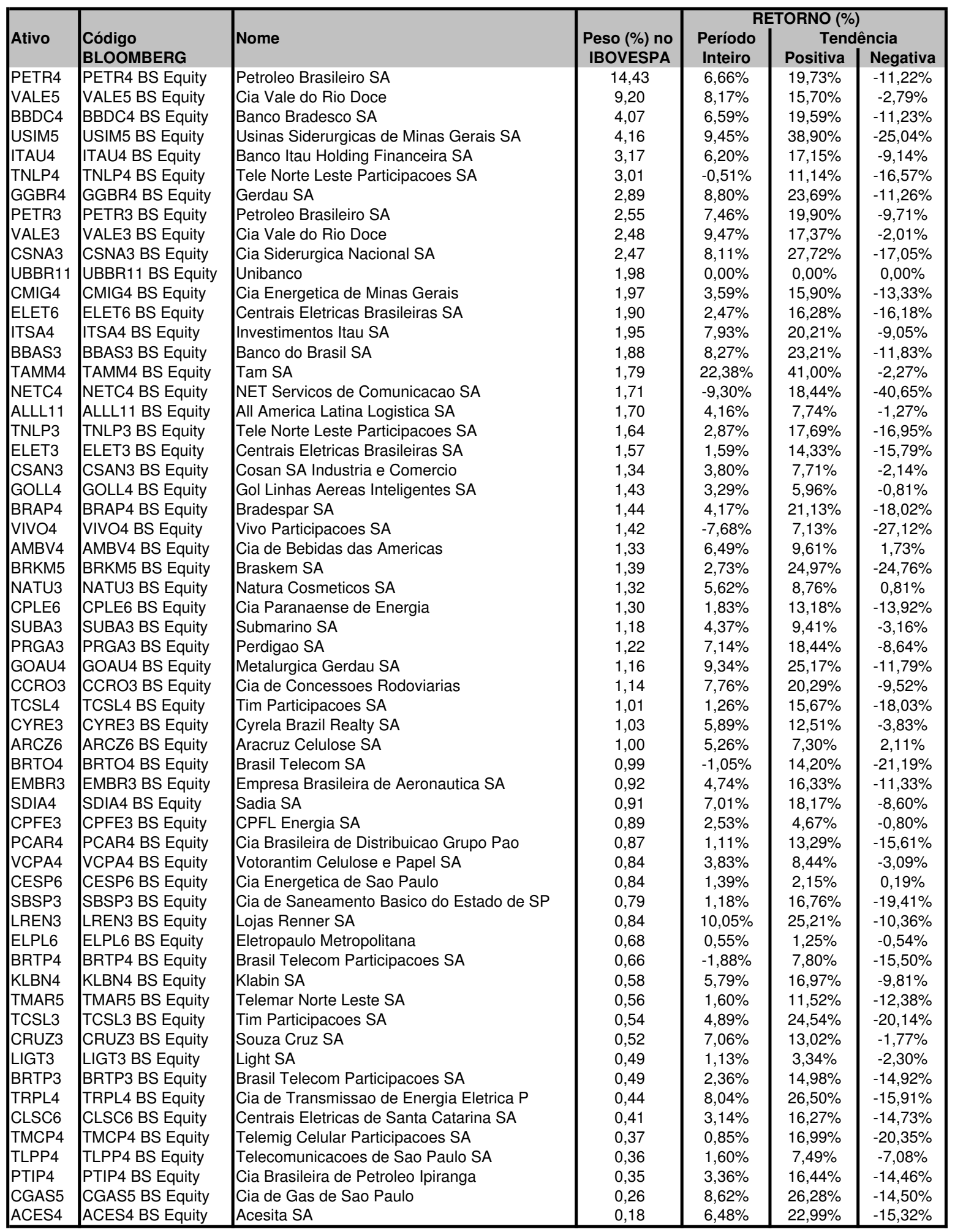

Tabela 7.20: Tabela com Ativos de Risco, seus códigos Bloomberg e retornos médios trimestrais. 

$\begin{array}{lllllllllllllllllllllllllllllllll}\text { SUBA3 } & 1,3 & 0,3 & 0,3 & 0,2 & 0,2 & 0,3 & 0,1 & 0,3 & 0,1 & 0,3 & 0,1 & 0,3 & 0,2 & 0,1 & 0,3 & 0,1 & 0,0 & 0,2 & 0,2 & 0,2 & 0,3 & 0,2 & 0,2 & 0,3 & 0,3 & 0,2 & 0,3 & 0,1 & 0,3 & 0,3 & 0,2\end{array}$

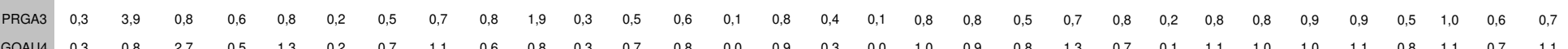
$\begin{array}{llllllllllllllllllllllllllllllllll}\text { GOAU4 } & 0,3 & 0,8 & 2,7 & 0,5 & 1,3 & 0,2 & 0,7 & 1,1 & 0,6 & 0,8 & 0,3 & 0,7 & 0,8 & 0,0 & 0,9 & 0,3 & 0,0 & 1,0 & 0,9 & 0,8 & 1,3 & 0,7 & 0,1 & 1,1 & 1,0 & 1,0 & 1,1 & 0,8 & 1,1 & 0,7 & 1,1\end{array}$ $\begin{array}{lllllllllllllllllllllllllllllllllll}\text { CCRO3 } & 0,2 & 0,6 & 0,5 & 3,4 & 0,5 & 0,2 & 0,2 & 0,6 & 0,1 & 0,5 & 0,1 & 0,4 & 0,1 & 0,1 & 0,5 & 0,2 & 0,0 & 0,6 & 0,4 & 0,5 & 0,3 & 0,3 & 0,1 & 0,5 & 0,5 & 0,6 & 0,5 & 0,2 & 0,5 & 0,3 & 0,3 \\ \text { TCSL4 } & 0,2 & 0,8 & 1,3 & 0,5 & 5,5 & 0,3 & 0,9 & 1,9 & 1,4 & 1,0 & 0,2 & 0,9 & 0,8 & 0,1 & 1,3 & 0,5 & 0,0 & 1,9 & 1,3 & 1,4 & 3,3 & 1,0 & 0,1 & 2,1 & 2.0 & 1,6 & 2,7 & 1,6 & 1,7 & 1,4 & 1,7\end{array}$ $\begin{array}{llllllllllllllllllllllllllllllllll}\text { CYRE3 } & 0,3 & 0,2 & 0,2 & 0,2 & 0,3 & 1,8 & 0,1 & 0,2 & 0,2 & 0,2 & 0,1 & 0,3 & 0,2 & 0,1 & 0,2 & 0,2 & 0,0 & 0,2 & 0,2 & 0,2 & 0,2 & 0,2 & 0,1 & 0,2 & 0,1 & 0,3 & 0,3 & 0,1 & 0,2 & 0,1 & 0,1\end{array}$ \begin{tabular}{llllllllllllllllllllllllllllllllll} 
ARCZ6 & 0,1 & 0,5 & 0,7 & 0,2 & 0,9 & 0,1 & 3,0 & 0,6 & 0,6 & 0,5 & 0,1 & 0,6 & 1,3 & 0,0 & 0,5 & 0,0 & 0,0 & 0,9 & 0,7 & 0,4 & 0,9 & 0,4 & 0,1 & 0,9 & 0,8 & 0,6 & 0,8 & 0,6 & 0,6 & 0,3 & 0,6 \\
\hline
\end{tabular} \begin{tabular}{lllllllllllllllllllllllllllllllllll} 
BRTO4 & 0,3 & 0,7 & 1,1 & 0,6 & 1,9 & 0,2 & 0,6 & 4,1 & 1,0 & 0,8 & 0,2 & 0,8 & 0,9 & 0,1 & 1,3 & 0,1 & 0,0 & 2,9 & 1,2 & 1,7 & 1,9 & 0,9 & 0,1 & 2,7 & 1,7 & 1,5 & 1,8 & 1,2 & 1,5 & 1,3 & 1,5 \\
\hline
\end{tabular} $\begin{array}{lllllllllllllllllllllllllllllllllll}\text { EMBR3 } & 0,1 & 0,8 & 0,6 & 0,1 & 1,4 & 0,2 & 0,6 & 1,0 & 4,1 & 0,7 & 0,1 & 0,6 & 0,8 & 0,0 & 0,7 & -0,2 & 0,0 & 1,0 & 0,8 & 0,6 & 1,4 & 0,6 & 0,1 & 1,1 & 1,1 & 0,9 & 1,3 & 0,8 & 1,0 & 0,6 & 1,1\end{array}$ \begin{tabular}{lllllllllllllllllllllllllllllllllll} 
SDIA4 & 0,3 & 1,9 & 0,8 & 0,5 & 1,0 & 0,2 & 0,5 & 0,8 & 0,7 & 3,4 & 0,3 & 0,6 & 0,5 & 0,1 & 0,8 & 0,4 & 0,1 & 0,8 & 0,8 & 0,6 & 1,0 & 0,8 & 0,2 & 0,9 & 0,9 & 1,1 & 0,6 & 0,6 & 0,9 & 0,7 & 0,9 \\
\hline
\end{tabular} \begin{tabular}{llllllllllllllllllllllllllllllllll} 
CPFE3 & 0,1 & 0,3 & 0,3 & 0,1 & 0,2 & 0,1 & 0,1 & 0,2 & 0,1 & 0,3 & 0,8 & 0,2 & 0,2 & 0,0 & 0,2 & 0,5 & 0,0 & 0,2 & 0,2 & 0,2 & 0,3 & 0,1 & 0,1 & 0,2 & 0,2 & 0,3 & 0,2 & 0,2 & 0,2 & 0,2 & 0,2 \\
\hline
\end{tabular} \begin{tabular}{lllllllllllllllllllllllllllllllllll} 
PCAR4 & 0,3 & 0,5 & 0,7 & 0,4 & 0,9 & 0,3 & 0,6 & 0,8 & 0,6 & 0,6 & 0,2 & 3,1 & 0,5 & 0,0 & 0,7 & 0,2 & 0,0 & 1,0 & 0,6 & 0,7 & 0,7 & 0,5 & 0,0 & 0,9 & 0,8 & 0,7 & 0,7 & 0,8 & 0,8 & 0,5 & 0,8 \\
\hline
\end{tabular} \begin{tabular}{lllllllllllllllllllllllllllllllll} 
VCPA4 & 0,2 & 0,6 & 0,8 & 0,1 & 0,8 & 0,2 & 1,3 & 0,9 & 0,8 & 0,5 & 0,2 & 0,5 & 2,8 & 0,0 & 0,5 & 0,1 & 0,0 & 0,9 & 0,7 & 0,6 & 0,8 & 0,6 & 0,1 & 0,9 & 0,8 & 0,8 & 0,7 & 0,6 & 0,9 & 0,5 & 0,8 \\
\hline
\end{tabular} \begin{tabular}{lllllllllllllllllllllllllllllllllll} 
CESP6 & 0,1 & 0,1 & 0,0 & 0,1 & 0,1 & 0,1 & 0,0 & 0,1 & 0,0 & 0,1 & 0,0 & 0,0 & 0,0 & 0,2 & 0,0 & 0,0 & 0,1 & 0,1 & 0,0 & 0,1 & 0,0 & 0,1 & 0,1 & 0,1 & 0,1 & 0,0 & 0,1 & 0,0 & 0,0 & 0,0 & 0,0 \\
\hline
\end{tabular} \begin{tabular}{lllllllllllllllllllllllllllllllllll} 
SBSP3 & 0,3 & 0,8 & 0,9 & 0,5 & 1,3 & 0,2 & 0,5 & 1,3 & 0,7 & 0,8 & 0,2 & 0,7 & 0,5 & 0,0 & 3,5 & 0,1 & 0,0 & 1,3 & 1,0 & 1,1 & 1,3 & 0,7 & 0,0 & 1,5 & 1,3 & 1,3 & 1,3 & 1,1 & 1,1 & 1,1 & 1,3 \\
\hline
\end{tabular} $\begin{array}{llllllllllllllllllllllllllllllllll}\text { LREN3 } & 0,1 & 0,4 & 0,3 & 0,2 & 0,5 & 0,2 & 0,0 & 0,1 & -0,2 & 0,4 & 0,5 & 0,2 & 0,1 & 0,0 & 0,1 & 16,3 & 0,0 & 0,2 & 0,6 & 0,4 & 0,2 & 0,2 & 0,0 & -0,1 & 0,1 & 0,0 & 0,2 & 0,2 & -0,1 & 0,4 & 0,2\end{array}$

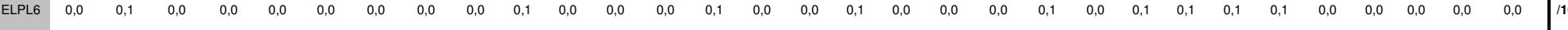
\begin{tabular}{llllllllllllllllllllllllllllllllll} 
& 0,2 & 0,8 & 1,0 & 0,6 & 1,9 & 0,2 & 0,9 & 2,9 & 1,0 & 0,8 & 0,2 & 1,0 & 0,9 & 0,1 & 1,3 & 0,2 & 0,0 & 4,0 & 1,2 & 1,5 & 1,8 & 0,9 & 0,1 & 3,0 & 1,3 & 1,4 & 2,0 & 1,2 & 1,3 & 1,2 & 1,4 \\
\hline
\end{tabular}

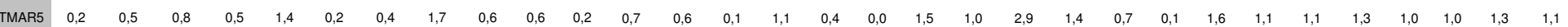

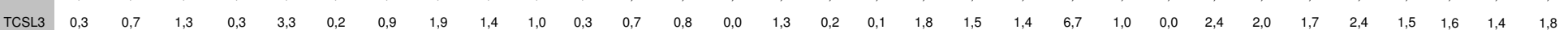

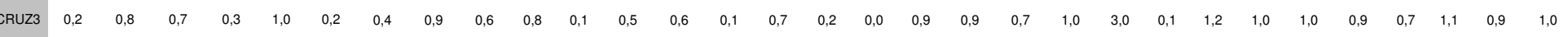

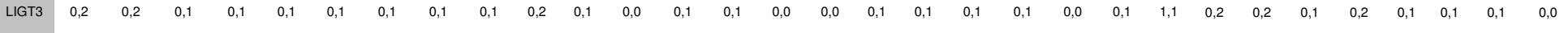
\begin{tabular}{llllllllllllllllllllllllllllllllllll} 
BRTP3 & 0,3 & 0,8 & 1,1 & 0,5 & 2,1 & 0,2 & 0,9 & 2,7 & 1,1 & 0,9 & 0,2 & 0,9 & 0,9 & 0,1 & 1,5 & $-0,1$ & 0,1 & 3,0 & 1,5 & 1,6 & 2,4 & 1,2 & 0,2 & 5,7 & 1,9 & 1,7 & 2,0 & 1,5 & 1,6 & 1,4 & 1,9 \\
\hline
\end{tabular} \begin{tabular}{lllllllllllllllllllllllllllllllllll} 
TRPL4 & 0,3 & 0,8 & 1,0 & 0,5 & 2,0 & 0,1 & 0,8 & 1,7 & 1,1 & 0,9 & 0,2 & 0,8 & 0,8 & 0,1 & 1,3 & 0,1 & 0,1 & 1,3 & 1,4 & 1,1 & 2,0 & 1,0 & 0,2 & 1,9 & 5,9 & 1,9 & 1,7 & 1,2 & 1,6 & 1,6 & 1,9 \\
\hline
\end{tabular} \begin{tabular}{lllllllllllllllllllllllllllllllllll}
\hline CLSC6 & 0,2 & 0,9 & 1,0 & 0,6 & 1,6 & 0,3 & 0,6 & 1,5 & 0,9 & 1,1 & 0,3 & 0,7 & 0,8 & 0,0 & 1,3 & 0,0 & 0,1 & 1,4 & 1,2 & 1,1 & 1,7 & 1,0 & 0,1 & 1,7 & 1,9 & 4,1 & 1,6 & 1,1 & 1,5 & 1,4 & 1,7 \\
\hline
\end{tabular}

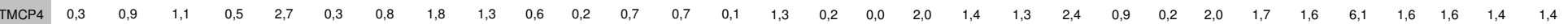
$\begin{array}{llllllllllllllllllllllllllllllllll}\text { TLPP4 } & 0,1 & 0,5 & 0,8 & 0,2 & 1,6 & 0,1 & 0,6 & 1,2 & 0,8 & 0,6 & 0,2 & 0,8 & 0,6 & 0,0 & 1,1 & 0,2 & 0,0 & 1,2 & 0,9 & 1,0 & 1,5 & 0,7 & 0,1 & 1,5 & 1,2 & 1,1 & 1,6 & 3,5 & 0,9 & 1,0 & 1,1\end{array}$ \begin{tabular}{llllllllllllllllllllllllllllllllll} 
PTIP4 & 0,3 & 1,0 & 1,1 & 0,5 & 1,7 & 0,2 & 0,6 & 1,5 & 1,0 & 0,9 & 0,2 & 0,8 & 0,9 & 0,0 & 1,1 & $-0,1$ & 0,0 & 1,3 & 1,2 & 1,0 & 1,6 & 1,1 & 0,1 & 1,6 & 1,6 & 1,5 & 1,6 & 0,9 & 4,2 & 1,2 & 1,7 \\
\hline
\end{tabular}

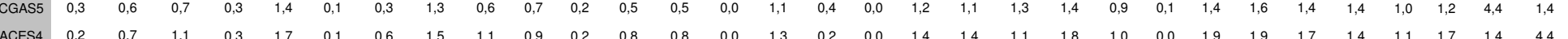
ACES4 


\section{Capítulo 8}

\section{Conclusão}

Investigou-se em tempo discreto o problema de otimização multi-período de carteiras generalizado em média-variância cujos coeficientes de mercado são modulados por uma cadeia de Markov finita $(P G M V)$. A solução para tal problema pode ser utilizada em uma grande variedade de problemas de média-variância, como os modelos que consideram em suas funções objetivo os valores intermediários da esperança ou variância do valor da carteira, ou em problemas com controle sobre o risco de falência, como formulado em Zhu et al. (2004) para o caso sem saltos. Foram derivadas condições necessárias e suficientes para obtenção da estratégia ótima do problema generalizado de média-variância, apresentando-se um algoritmo para obtenção de cada uma delas. Demonstrou-se que a lei de controle ótima depende da solução de um conjunto de equações a diferenças de Riccati interconectadas (4.2) e em um segundo conjunto de equações recursivas (4.34) e (4.35). Expressões analíticas foram derivadas para os problemas de média-variância, associados ao problema generalizado, em cujos modelos as restrições incidem apenas no instante final. Comprovou-se que as soluções aqui encontradas, quando particularizadas para a condição em que a função objetivo e as restrições incidam apenas sobre o instante final, coincidem com as apresentadas 
em Çakmak e Özekici (2006). Adicionalmente, demonstrou-se como estender o modelo $P G M V$ para solução do modelo em multi-período de seleção de carteiras generalizado de média-variância do tracking error, cujos parâmetros de mercado estão sujeitos a saltos Markovianos (PGMVTE). Demonstrou-se como adaptar as soluções encontradas para os modelos de média-variância associados ao modelo $P G M V$, na solução dos problemas de média-variância do tracking error, derivados do problema PGMVTE. Ilustrou-se, com dados reais, a aplicação das soluções aqui encontradas, onde se apresentou que os resultados dos problemas cujos parâmetros estão sujeitos a saltos Markovianos superam os modelos que não possuem esta característica. Como sugestão de futuras pesquisas, indica-se a inserção de restrições ao modelo, como a de venda a descoberto, que poderiam tornar os portfólios obtidos mais reais. 


\section{Referências Bibliográficas}

Alexander, G. J. e Baptista, A. M. (2005). Active portfolio management with benchmarking: Adding a value-at-risk constraint. Working Paper - University of Minnesota.

Ammann, M. e Zimmermann, H. (2001). Tracking error and tactical asset allocation. Financial Analysts Journal, 57:32-43.

Araujo, M. e Costa, O. L. V. (2006a). Discrete-time mean-variance portfolio optimization with Markov switching parameters. Proceedings of the 2006 American Control Conference, pp. 917-922.

Araujo, M. e Costa, O. L. V. (2006b). Otimização de carteiras por média-variância a tempo discreto e sujeitas a saltos Markovianos. Anais do XVI Congresso Brasileiro de Automática, pp. 709-714.

Artzner, P.; Delbaen, F.; Eber, J.-M. e Heath, D. (1999). Coherent measures of risk. Math Finance, 9:203-227.

Bauerle, N. e Rieder, U. (2004). Portfolio optimization with Markov-modulated stock prices and interest rates. IEEE Trans. Autom. Control, 49:442-447.

Bernoulli, D. (1954). Exposition of a new theory on the measurement of risk. Econometrica, 22:23-36. 
Bielecki, T. R.; Jin, H.; Pliska, S. R. e Zhou, X. Y. (2005). Continuous-time meanvariance portfolio selection with bankruptcy prohibition. Mathematical Finance, $15: 213-244$.

Brennan, M. J. (1993). Agency and asset pricing. Anderson Scchool of Management$U C L A$.

Browne, S. (1999). Beating a moving target: Optimal portfolio strategies for outperforming a stochastic benchmark. Finance and Stochastics, 3:275-294.

Buffington, J. e Elliott, R. (2002). American options with regime switching. Int. J. Theor. Appl. Finance, 5:497-514.

Cajueiro, D. O. (2002). Stochastic Optimal Control of jumping Markov Parameter Processes with Applications to Finance. PhD thesis, Instituto Tecnologico de Aeronautica.

Campbell, J. Y. e Viceira, L. M. (2001). Strategic Asset Allocation: portfolio Choice for Long-term Investors. Willey.

Çakmak, U. e Özekici, S. (2006). Portfolio optimization in stochastic markets. Math. Methods Oper. Research, 63:151-168.

Chow, G. (1995). Portfolio selection based on return, risk and relative performance. Financial Analysts Journal, 51:54-60.

Costa, O. L. V. e Araujo, M. (2007a). A generalized multi-period mean-variance portfolio optimization with Markov switching parameters. Paper submetido a periódico internacional.

Costa, O. L. V. e Araujo, M. (2007b). Um modelo generalizado de otimização de carteiras em média-variância com saltos Markovianos. Anais do VII Encontro Brasileiro de Finanças, pp. 1614-1636. 
Costa, O. L. V.; Fragoso, M. D. e Marques, R. P. (2005). Discrete-Time Markov Jump Linear Systems. Springer-Verlag.

Costa, O. L. V. e Nabholz, R. (2005). A multi-period mean-variance portfolio selection problem. Revista Brasileira de Finanças, 1:101-121.

Costa, O. L. V. e Paiva, A. C. (2002). Robust portfolio selection using linear matrix inequalities. Journal of Economic Dynamics and Control, 26:889-909.

Dumas, B. e Luciano, E. (1991). An exact solution to a dynamic portfolio choice problem under transaction costs. Journal of Finance, 46:577-595.

Fabozzi, F. J.; Jones, F. J. e Vardharaj, R. (2004). Determinants of tracking error for equity portfolios. The Journal of Investing.

Fabozzi, F. J.; Kolm, P. N.; Pachamanova, D. e Focardi, S. M. (2007). Robust Portfolio Optimization and Management. Wiley.

Fouque, J.; Papanicolaou, G. e Sircar, K. (2000). Derivatives in Financial Markets with Stochastic Volatility. Cambridge Univ. Press.

Goldsmith, D. (1976). Transactions costs and the theory of portfolio selection. Journal of Finance, 31:1127-1139.

Gressis, N.; Philippatos, G. e Hayya, J. (1976). Multiperiod portfolio analysis and the inefficiency of the market portfolio. Journal of Finance, 31:1115-1125.

Hull, J. C. (1997). Options, Futures and Other Derivatives. Prentice-Hall.

Ingersoll, J. E. (1987). Theory of Financial Decision Making. Rowman Littlefield.

Jorion, P. (2003). Portfolio optimization with tracking-error constraints. Financial Analysts Journal, 59:70-82.

Karatzas, I. (1997). Lectures on the Mathematics of Finance. American Mathematical Society, Providence. 
Leippold, M.; Trojani, F. e Vanini, P. (2004). A geometric approach to multiperiod mean variance optimization of assets and liabilities. Journal of Economic Dynamic Control, 28:1079-1113.

Li, D. e Ng, W. (2000). Optimal dynamic portfolio selection: Multi-period meanvariance formulation. Math. Finance, 10:387-406.

Lim, A. e Zhou, X. Y. (2002). Mean-variance portfolio selection with random coefficients in a complete market. Math. Oper. Res., 27:101-120.

Markowitz, H. (1952). Portfolio selection. J. Finance, 7:77-91.

Markowitz, H. (1970). Portfolio Selection: Efficient Diversification of Investments. Willey, Yale University Press.

Markowitz, H. (1999). The early history of portfolio theory: 1600-1960. Financial Analysts Journal, 55:5-16.

Markowitz, H. (2000). Mean-Variance Analysis in Portfolio Choice and Capital Markets. Basis Blackwell.

Masi, G. D.; Kabanov, Y. e Runggaldier, W. (1994). Mean variance hedging of options with Markov volatility. Theory Probab. Appl., 39:173-181.

Merton, R. C. (1969). Lifetime portfolio selection under uncertainty: The continuous-time case. Review of Economics and Statistics, 51:247-257.

Mossin, J. (1968). Optimal multi-period portfolio policies. Journal of Business, 41:215229.

Ortobelli, S.; Rachev, S. T.; Stoyanov, S.; Fabozzi, F. J. e Biglova, A. (2005). The proper use of risk measures in portfolio theory. International Journal of Theoretical and Applied Finance, pp. 1107-1133.

Pliska, S. (1997). Introduction to Mathemaical Finance. Oxford: Blackwell. 
Pratt, J. (1964). Risk aversion in the small and in the large. Econometrica, 32:122-136.

Rami, M. A.; Chen, X. e Zhou, X. (2002). Discrete-time indefinite lq control with state and control dependent noises. Journal of Global Optimization, 23:245-265.

Rockafellar, R. T.; Uryasev, S. e Zabarankin, M. (2006). Generalized deviations in risk analysis. Finance and Stochastic, 10:51-74.

Roll, R. (1992). A mean/variance analysis of tracking error. Journal of Portfolio Management, 18:13-22.

Roy, A. (1952). Safety first and the holding of assets. Econometrica, 20:431-449.

Saberi, A.; Sannuti, P. e Chen, B. M. (1995). $H_{2}$-Optimal Control. Prentice-Hall.

Samuelson, P. A. (1969). Lifetime portfolio selection by dynamic stochastic programming. Review of Economics and Statistics, 51:239-246.

Tobin, J. (1965). The Teory of Portfolio Selection. Macmillan.

Yin, G. e Zhou, X. Y. (2004). Markowitz's mean-variance portfolio selection with regime switching: From discrete-time models to their continuous-time limits. IEEE Trans. Autom. Control, 49:349-360.

Zhang, Q. (2000). Stock trading: An optimal selling rule. SIAM J. Control Optim., 40:64-87.

Zhao, Y. e Ziemba, W. T. (2000). Mean-variance versus expected utility in dynamic investment analysis. University of Britsh Columbia, pp. 1-29.

Zhou, X. Y. e Li, D. (2000). Continuous-time mean-variance portfolio selection: A stochastic LQ framework. Appl. Math. Optim., 42:19-33.

Zhou, X. Y. e Yin, G. (2003). Markowitz's mean-variance portfolio selection with regime switching: A continuous-time model. SIAM J. Control Optim., 42:14661482. 
Zhu, S.-S.; Li, D. e Wang, S.-Y. (2004). Risk control over bankruptcy in dynamic portfolio selection: A generalized mean-variance formulation. IEEE Trans. Autom. Control, 49:447-451. 